\title{
UM MÉTODO DE CONFUNDIMENTO NOS EXPERIMENTOS FATORIAIS
}

\author{
ELIO PAULO ZOHTA \\ Engenheiro Agrônomo
}

Orientador: DR. HUMBERTO DE CAMPOS

Dissertação apresentada a Escola Superior de Agriculti "Luiz de Queiróz", da Universidade de São Paulo, para obłençāo do titulo de Mestre em Estatistica e Experim taçôo Agronômica.

PIRACICABA

Estado de São Paulo - Brasil

Marco, 1980 


\title{
UM MÉTODO DE CONFUNDIMENTO NOS EXPERIMENTOS FATORIAIS
}

\author{
ELLO PAULO ZONTA \\ Engenheiro Agrônomo
}

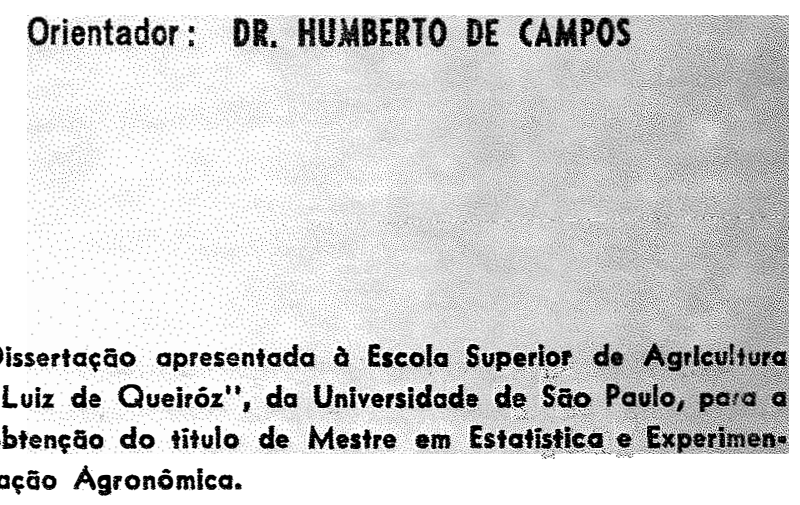

PIRACI CABA

Estado de São Paulo - Brasll

Março, 1980 
A meus pais,

à minha esposa Mariza,

e a minha fllha Mabel

$D E D \perp C O$ 


\section{AGRADECIMENTOS}

- Ao professor Dr. Humberto de Campos.

- Ao professor Di. Frederico Pimentel Gomes.

- Aos professores e funcionários do Departa-

mento de Matemática e Estatística da ESAL 2 .

- Ao professor Dr. Paulo Silveira Jünior.

- Ao professor Dr. Edilberto Amaral.

- Aos professores e funcionários de Departamento de Matemätica e Estatística da UFPel.

- Ao professor Mário Capanema Ulyssea.

. Ao professor Clóvis de Almeida Alt.

- Ao Núcleo de Processamento de Dados da Uni-

versidade Federal de Pelotas.

- Ao Professor Paulo Gomes da Silva.

- Aos colegas do Curso de Estatística e Experimentação Agrônomica.

- A todos os que contribuiram para a realização do curso e deste trabalho. 
1. RESUMO...........................

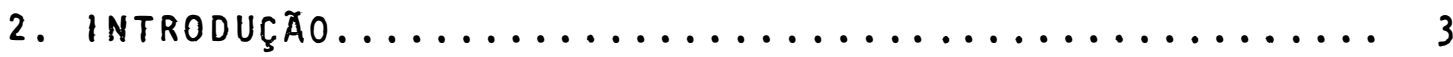

3. REVISÃo de LITERATURA..................... 7

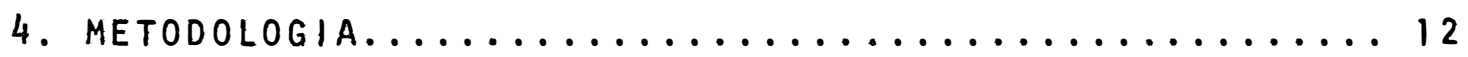

4.1. Confundimento da série $2^{n} \ldots \ldots \ldots \ldots \ldots \ldots \ldots$

4.1.1. Escolha das interações a serem confundidas......................... 13

4.1.2. Construção das tabelas bäsicas........ 14 4.1.2.1. Efeitos fatoriais com nümero impar de fatores.......... 15 4.1.2.2. Interações com nümero par de fatores............... 16

4.1.3. Formação dos blocos de cada repetição... 17 4.1.4. Formação de blocos de duas unidades..... 21 4.1.5. Construção de fatoriais balanceados..... 22 4.1 .5 .1 . Fatorial $2^{2} \ldots \ldots \ldots \ldots 23$ 4.1 .5 .2 . Fatorial $2^{3} \ldots \ldots \ldots \ldots 23$ 4.1.5.3. Fatorial $2^{4} \ldots \ldots \ldots \ldots \ldots 24$ 4.1.5.4. Fatorial $2^{5} \ldots \ldots \ldots \ldots 25$

4.1.6. Propriedades das tabelas bäsicas...... 27 4.2. Confundimento da sërie $3^{n} \ldots \ldots \ldots \ldots \ldots \ldots \ldots \ldots \ldots$

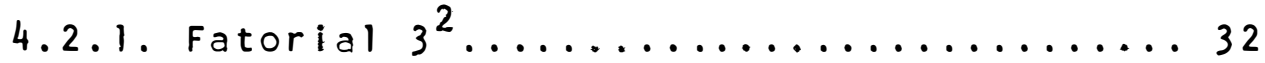


Pàg.

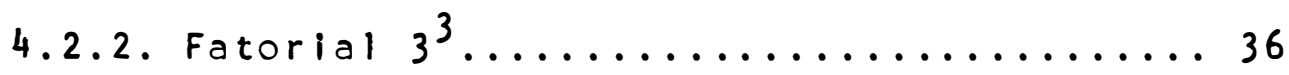

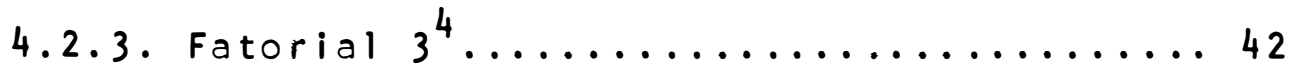

4.3. Confundimento da sërie $3 \times 2^{n} \ldots \ldots \ldots \ldots \ldots 5$

4.3.1. Fatorial $3 \times 2 \ldots \ldots \ldots \ldots \ldots \ldots \ldots$

4.3.2. Fatorial $3 \times 2^{2} \ldots \ldots \ldots \ldots \ldots \ldots \ldots$

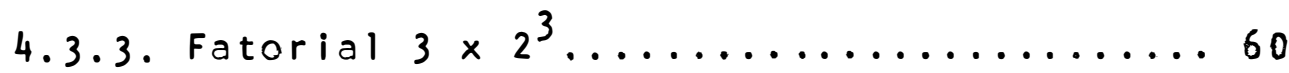

4.4. Confundimento da série $3^{2} \times 2^{n} \ldots \ldots \ldots \ldots \ldots$

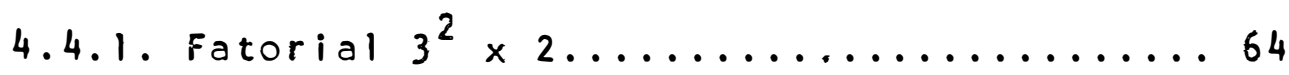

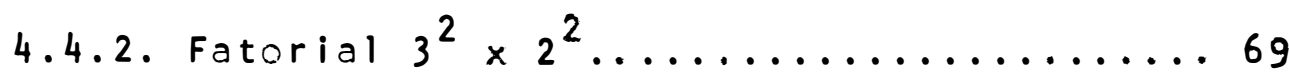

4.5. Confundimento da série $3^{3} \times 2^{n} \ldots \ldots \ldots \ldots \ldots$

4.5.1. Fatorial $3^{3} \times 2 \ldots \ldots \ldots \ldots \ldots \ldots \ldots$

4.6. Processo de obtenção da informação relativa.... 82

4.6.1. Sistema de equações normais para efeitos de tratamentos estimados............8 82

4.6.2. Obtenção da matriz de dispersão....... 84

4.6.3. Variâncias dos efeitos fatoriais.......87

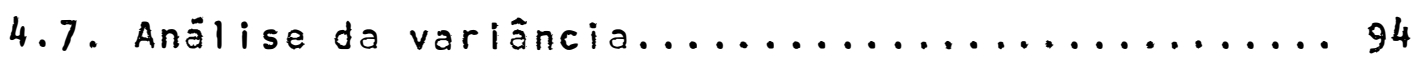

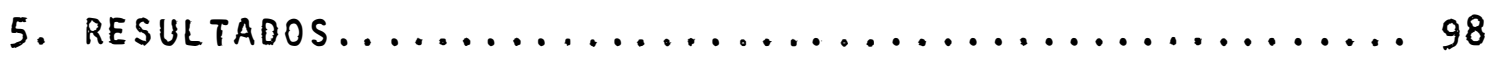

5.1. Fatoriais balanceados da sērie $2^{n} \ldots \ldots \ldots \ldots$

5.1 .1 . Fatorial $2^{2} \ldots \ldots \ldots \ldots \ldots \ldots \ldots$

5.1.2. Fatorial $2^{3} \ldots \ldots \ldots \ldots \ldots \ldots \ldots$

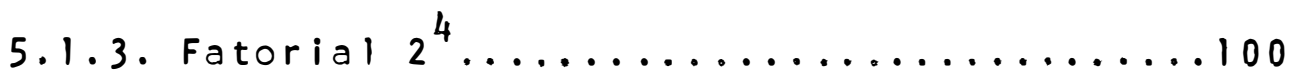

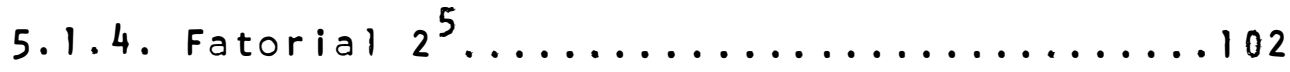


päg.

5.2. Facorialis balonceados da sério $3^{n} \ldots \ldots \ldots \ldots 108$ 5.2.1. Fatorial $3^{2} \ldots \ldots \ldots \ldots \ldots \ldots \ldots$ 5.2.2. Fatorial $3^{3} \ldots \ldots \ldots \ldots \ldots \ldots$ 5.2.3. Fatorial $3^{4} \ldots \ldots \ldots \ldots \ldots \ldots \ldots \ldots \ldots$

3.3. Fatorials balanceados da sërie $3 \times 2^{n} \ldots \ldots \ldots$ 2?

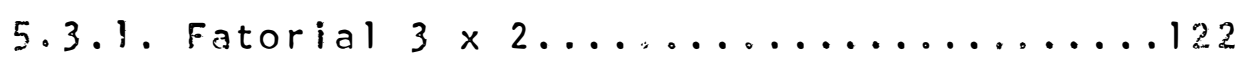

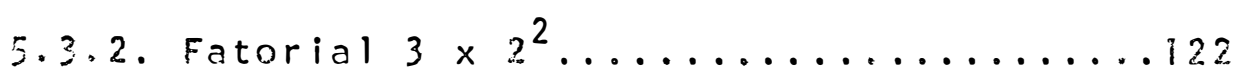

5.3 .3 . Fatorial $3 \times 2^{3} \ldots \ldots \ldots \ldots \ldots \ldots \ldots \ldots$

5.4. Fatoriais balanceados da sërie $3^{2} \times 2^{n} \ldots \ldots \ldots 126$

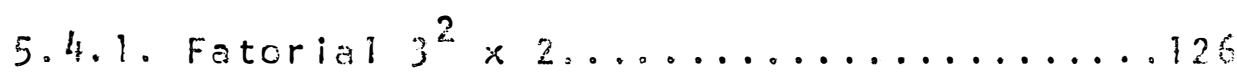
5.4.2. Fatorial $3^{?} \times 2^{2} \ldots \ldots \ldots \ldots \ldots \ldots$

5.5. Fatoriais balanceados de sërie $3^{3} \times 2^{n} \ldots \ldots \ldots$ 5.5 .1 . Fatoriai $3^{3} \times 2 \ldots \ldots \ldots \ldots \ldots \ldots \ldots$

S. DIscussño............................

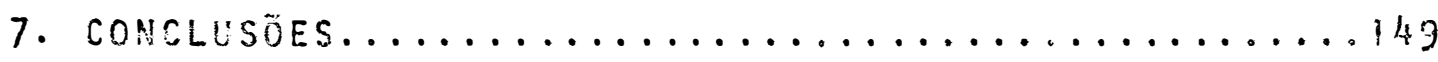

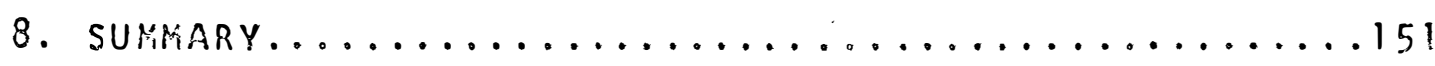

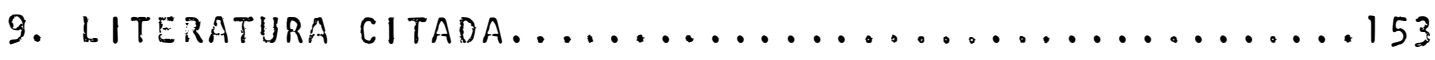

10. APENDICE 1 - Instruções para o uso do programa de obtenção da informação relativa.....154

11. APENDICE 2 - Listagem do programa, da subrotina PRDD e exemplo de aplicação......... 159 
1. RESUMO

0 presente trabalho estabelece um processo de confundimento para os fatoriais da sērie $2^{n}$ e, através de duas propriedades deste, torna possivel o confundimento' de fatoriais com qualquer número de niveis e de fatores.

Foram estudados os fatoriais com dois e três niveis, ou sejam, as sèries $2^{n}, 3^{n}, 3 \times 2^{n}, 3^{2} \times 2^{n}$ e $3^{3} \times 2^{n}$.

Para cada fatorial da sērie $2^{n}$ foram cons. truidos conjuntos balanceados até o fatorial $2^{5}$, com a subdivisão das repetições em dois blocos, até a formação de blocos de duas unidades, sem o confundimento de efeitos ' principais. 
2.

Na sèrie $3^{n}$, foram construídos conjuntos' balanceados para os fatoriais $3^{2}, 3^{3}$ e $3^{4}$, dividindo-se as repetiçōes em três blocos, no fatorial $3^{2}$, blocos de nove e de três unidades no fatorial $3^{3}$ e, no fatorial $3^{4}$, blocos de vinte e sete, de nove e de três unidades.

Nas sēries mistas, foram estudados os fato riais $3 \times 2,3 \times 2^{2}$ e $3 \times 2^{3}$, da sērie $3 \times 2^{n}$; os fatoriais $3^{2} \times 2$ e $3^{2} \times 2^{2}$, da sèrie $3^{2} \times 2^{n}$ e, finalmente, o fatorial $3^{3} \times 2$, da sèrie $3^{3} \times 2^{n}$.

Alëm do mètodo de confundimento è dado o processo de obtenção da informação relativa atravēs da teoria dos blocos incompletos e o método geral de anälise da variância . 
2. INTRODUÇÃO

Experimentos fatoriais são aqueles em que os tratamentos resultam de todas as combinações possiveis' dos niveis dos diversos fatores. Assim, por exemplo, em um experimento de adubação com nitrogênio (N) e fösforo (P), os fatores são $N$ e $P$, os niveis, as suas doses. Os tratamentos provêm das combinações dos níveis $N$ e $P$. Tomando-se três do ses de cada fator, tem-se um fatorial $3^{2}$ com nove combina f̧ões ou nove tratamentos. Com mais um fator, potássio (K), por exemplo, também com três níveis, resulta um fatorial $3^{3}$ com 27 tratamentos, os quais, se forem dispostos em um delineamento de blocos ao acaso, em um experimento de campo tornarão as repetições excessivamente grandes, tendo, poR consequência, a diminuição da precisão do experimento. 
Neste trabalho, sempre que houver referência a repetições, estas serão provenientes do delineamento' em blocos ao acaso.

Na anălise da variância de um fatorial $3^{3}$, como descrito anteriormente, tem-se 26 graus de liberdade para tratamentos, dos quais seis são atribuidos aos efeitos de $N, P$ e K, denominados de efeitos principais. Além desses efeitos, poderá haver influências de um fator sobre o outro sendo então denominadas de interações de dois fatores, que neste caso são: NP, NK e PK, cada uma com quatro graus de liberdade. Interações de dois fatores são tambèm conhecidas como interações de primeira ordem. Tem-se ainda, uma intera ção de três fatores, ou de segunda ordem, a qual é mais dificil de ser interpretada, podendo ser, a influência de um fator sobre os outros, isoladamente, ou a influência de um deles sobre a interação dos outros dois. A esta interação' estão associados os oito graus de liberdade restantes.

Cada um dos efeitos principais e intera ções è chamado de efeito fatorial.

No caso de fatoriais com grande nümero de tratamentos, que tornam as repetições excessivamente gran des, como o fatorial $3^{3}$, pode-se subdividi-las em dois ou mais blocos, dispondo-se parte dos tratamentos em cada um. Se uma repetição for dividida em $\underline{b}$ blocos, não é possfrel' estimar, nesta, $(b-1)$ graus de liberdade dos efeitos fatori 
ais, os quais ficam confundidos com os de blocos. Esses gra us de liberdade dependem diretamente dos tratamentos alocados em cada bloco. Logo, deve-se distribui-los de tal forma que os efeitos que não possam ser estimados, sejam os das interações de mais alta ordem. Assim, no fatorial $3^{3}$, pode- se, em cada repetição, formar três blocos de nove tratamen tos, os quais em ensaios agrícolas são geralmente de tama nho razoāvel, para dar uma boa precisão ao experimento. As combinaçöes dos niveis dos fatores são dispostas nos três blocos, de tal modo que os dois graus de liberdade que ficam confundidos com os de blocos sejam da interação de segunda ordem. Como esta possui oito graus de liberdade, hä quatro maneiras distintas de combinar os 27 tratamentos em três grupos de nove. Os dois graus de liberdade confundidos em cada repetição são recuperados nas outras, de tal forma que não hã perda dos mesmos.

A tëcnica de subdividir as repetições de um experimento fatorial em dois ou mais blocos, obter os efeitos fatoriais que não podem ser estimados, no todo ou em parte, e determinar os tratamentos que constituirão cada bloco, é denominada de confundimento. Diz-se então que, : subdividindo-se uma repetição em b blocos, tem-se nesta, ' (b-1) graus de liberdade confundidos com efeitos de blocos. 
Ouando todos os fatores apresentam o mesmo nümero de níveis, o confundimento è, geralmente, fäcil de se obter e seus esquemas são encontrados na literatura. Porëm, quando os fatores apresentam níveis diferentes, a literatura è mais escassa e muitas vezes o experimentador' è levado a alterar seu esquema inicial, devido a dificulda de encontrada na sua construção. Essa alteração poderà ' provocar profundas modificações nos objetivos do experimen to.

Desenvolveu-se então, um método sistemätico de confundimento dos fatoriais, que torna nossivel confundir fatoriais com qualquer nümero de niveis ou de fatores. 
3. REVISÃO DE LITERATURA

YATES (1937), apresenta os confundimentos total e parcial do fatorial $2^{3}$ em repetições com dois blocos de quatro parcelas cada, onde, em cada repetição, à confundido o efeito de blocos com o de uma das interações, tomando-se, no contraste correspondente, as combinações. com sinal (+) num bloco e as com sinal (-) no outro.

A partir do fatorial $2^{3}$ generaliza a sērie $2^{\text {n }}$ iniciando' pelo confundimento do fatorial $2^{4}$ em blocos de quatro parcelas, ofatorial $2^{5}$ em blocos de -8 e de 4 unidades $e$ fatorial $2^{6}$ em blocos de 16 , de 8 e de 4 tratamentos, e mostra o melhor meio de escolha das interações a serem con fundidas com blocos. 
Confunde, tambèm, o fatorial $2^{3}$ em quadra dos latinos $4 \times 4$ e os fatoriais $2^{5}$ e $2^{6}$ em quadrados latinos $8 \times 8$.

A partir do quadrado greco-latino $3 \times 3$, fetua o confundimento do fatorial $3^{2}$ em três blocos de. très parcelas. Para o fatorial $3^{3}$, apresenta os quatro gru pos $W, X, Y$ e $Z$, sem mostrar o processo utilizado na sua construção. Dá o esquema do fatorial $3^{4}$ em blocos de 9 par celas e a generalização da sērie $3^{n}$ em blocos de $3^{n-1}$ e $3^{n-2}$ unidades. A seguir apresenta os fatoriais $3^{3}$ e $3^{4}$ con fundidos em quadrados quasi-latinos.

Na sërie $3 \times 2^{n}$, apresenta os esquemas' dos fatoriais $3 \times 2^{2} \odot 3 \times 2^{3}$, confundidos em blocos de seis tratamentos e a sua generalização, em blocos de $3 \times 2^{n-1}$ e $3 \times 2^{n-2}$ unidades. Confunde, em função sa sërie $3^{n}$, os fatoriais $3^{2} \times 2$ e $3^{3} \times 2$, em blocos de $3^{n-1} \times 2$ e $3^{n-2} \times 2$ parcelas. Para o fatorial $3^{2} \times 2$ apresenta o esquema con fundido em um quadrado quasi-latino $6 \times 6$.

Finalmente, exfõe o mëtodo de confundimento de fatoriais com 4 e 8 niveis com base na sërie $2^{n}$.

Contudo, nesse trabalho, Yates, preocupa- se mais com a anälise estatística desses fatoriais, e não menciona nenhum método sistemätico de confundimento. 
FINNEY (1947) descreve um método sistemático de confundimento da sërie $2^{n}$, escolhe uma das inte. rações a serem confundidas com blocos e, pela combinação, das letras desta, determina os tratamentos do bloco conten do a testemunha (nivel inferior de todos os fatores), denomina_o de "bloco principal", e conströi através deste, os demais blocos. Por extensão deste processo confunde a sërie $3^{n}$.

Porèm, como o pröprio autor indica, tal processo não pode ser empregado diretamente no confundimento de outros fatoriais, cujo trabalho limita-se apenas às ' sèries $2^{n}$ e $3^{n}$.

COCHRAN e coX (1950) estudam o confundimen to total e parcial do fatorial $2^{3}$, confundindo contrastes. de interações com blocos, citando apōs, regras para a construção de fatoriais confundidos da série $2^{n}$.

Para a série $3^{\text {n }}$ apresentam o mëtodo de con fundimento do fatorial $3^{2}$, através do quadrado greco-latino $3 \times 3$, resultando dois grupos (duas repetições), cada um com três blocos de três parcelas. A partir do fatorial $3^{2}$, estruturam o confundimento do fatorial $3^{3}$ em blocos de nava unidades.

Nas sēries mistas, dão o processo de con fundimento do fatorial $3^{2} \times 2$, em blocos de 6 unidades, com 
binando primeiramente os niveis de $B$ e $C$ e $\because$, incluindo convenientemente os três niveis do fator $A$.

Mostram seis esquemas de fatoriais confun didos da s:-ie $2^{n}$ sendo: fatorial $2^{3}$ em blocos de 4 parcelas; $2^{4}$ em blocos de 8 e de 4 unidades; $2^{5}$ em blocos de 8 e o fatorial $2^{6}$ em blocos de 16 e 8 tratamentos. Com relação à sẻrie $3^{n}$, dão os esquemas' dos fatoriais $3^{3}$ e $3^{4}$ em blocos de nove parcelas e, para a sërie $4^{n}$, apresentam o esquema do fatorial $4^{2}$, confundido em quatro blocos de quatro unidades.

Nas sëries mistas, constroem os esquemas' dos fatoriais $3 \times 2^{2}, 3 \times 2^{3}$ e $3^{2} \times 2$, todos em blocos de seis tratamentos e o fatorial $4 \times 3 \times 2$ em blocos de 12 unidades.

Porëm, exceto para as séries $2^{n}$ e $3^{n}$, não dão o processo utilizado na obtenção dos confundimentos.

KEMPTHORNE (1952) apresenta um processo' de confundimento para a sërie $2^{n}$ e o exemplifica com o con fundimento parcial do fatorial $2^{3}$ em blocos de duas unidades e fatorial $2^{7}$ em blocos de 16 parcelas.

Apresenta também o plano com o confundi mento parcial do fatorial $2^{3}$ com quatro repetiçöes, confun dindo em cada uma as interações $A B, A C, B C$ e $A B C$. Efetua o confundimento to fatorial $2^{4}$ em blocos de quatro e de duas 
parcelas, todos no delineamento de blocos ao acaso. Determ $\underline{\underline{B}}$ na o confundimento do fatorial $2^{3}$ em quadrados latinos $4 \times 4$ e os fatoriais $2^{5}$ e $2^{6}$ em quadrados latinos $8 \times 8$. Com os fatores em três níveis, determina o confundimento através da geometria finita e apresenta as interações confundidas dos fatoriais $\operatorname{com} 2,3,4$ e 5 fato res.

Tambēm, atravēs da geometria finita, dá o processo geral de confundimento das sëries do tipo $p^{n}$, onde p è necessariamente um nümero primo.

No caso de fatoriais com dois e três ní veis apresenta os planos dos fatoriais $3 \times 2^{2}, 3^{2} \times 2$ e $3^{3} \times 2$, em blocos de seis unidades. 


\section{METODOLOGIA}

\subsection{Confundimento da sërte $2^{n}$}

$$
N a \text { sërie } 2^{n} \text {, o confundimento de um ou mais }
$$
efeitos fatoriais, geralmente interações de dois ou mais fa tores, é feito subdividindo-se as repetições em dois blocos ou em potência de dois.

Para que o confundimento seja eficiente hä necessidade de se confundir as interações de ordem mais ele vada. A seguir, ë dado um mëtodo de confundimento desta sërie, o qual consta de três etapas: escolha das interações a serem confundidas, construção de tabelas bäsicas e formação dos blocos por repetição. 


\subsubsection{Escolha das 1nteraçẽes a serem confundidas}

A escolha das interações é feita em função do número de blocos de cada repetição e do nümero de fato res envolvidos. Se uma repetição for dividida em b blocos, tem-se (b-l) graus de liberdade confundidos, que na série' $2^{n}$ correspondem a $(b-1)$ efeitos fatoriais. E dado a seguir os casos de b $=2,4$ e 8 .

\section{a) Subdivisão $\mathrm{mm}$ dols blocos}

Confunde-se sempre a interação de mais alta ordem, ou seja, a de $\underline{n}$ fatores, em todas as repetições.

b) Subdivisão em quatro blocos

Toma-se uma interação de $(n-1)$ fatores e através desta, determina-se as outras duas. Assim, por exem plo, escolhendo-se a interação $A B C$ do fatorial $2^{4}$, as outras duas interações poderão ser determinadas com o auxilio do outro fator da seguinte forma:

$$
\begin{aligned}
& (A+B C) D=A D+B C D, O U \\
& (B+A C) D=B D+A C D, O U \\
& (C+A B) D=C D+A B D
\end{aligned}
$$

Portanto, para subdividir uma repetição em quatro blocos, se escolnida a interação $A B C$ do fatorial $2^{4}$, as interações confundidas serão: 


$$
\begin{aligned}
& A B C, A D \text { e } B C D ; O U \\
& A B C, B D \text { e } A C D ; O U \\
& A B C, C D \text { e } A B D .
\end{aligned}
$$

\section{c) Subdivisão em oito blocos}

A partir de uma interação de $(n-2)$ fatores determina-se as outras seis. Assim, por exemplo, escolhendo - se a interação $A B C$ do fatorial $2^{5}$, as outras seis intera ções são obtidas com o auxilio dos outros dois fatores, de E, procedendo-se da seguinte forma:

$$
\begin{aligned}
& (A+B C) D=A D+B C D, \\
& (B+A C) E=B E+A C E, \\
& (C+A B) D E=C D E+A B D E . \\
& \text { Logo, as interaçöes que resultam confundi- }
\end{aligned}
$$
das são: $A D, B E, A B C, A C E, B C D, C D E$ e $A B D E$.

Outros grupos de interações poderão ser ob tidos desde que se permute as posições de $D, E$ e $D E$ ao se realizar o produto com a interação $A B C$.

\subsubsection{Construção das tabelas básicas}

Qualquer efeito fatorial da sërie $2^{n}$ pode ser obtido através de um contraste entre todas as combina ções dos fatores, cujos coeficientes são +1 e -1. Dä-se, a seguir,as regras para a determinação das combinações com coeficientes +1 e -1 . 
4.1.2.1. Efeitos fatoriais com número Impar de fatores

a) Combinações com sinal positivo

Forma-se uma tabela de dupla entrada, onde na horizontal (denominada de "horizontal bäsica") são colocadas todas as combinações impares possiveis entre os fatores do efeito fatorial a ser confundido e, na vertical (denominada "vertical băsica"), as combinações entre os fatores restantes, ou sejam, aqueles que não entraram na composição c reito fatorial enfocado, iniciando-se pela testemunha. Completa-se a tabela, tomando-se o produto com cada uma das combinações que aparecem nas colunas da horizontal' bäsica. Assim procedendo obtēm-se as combinações do efeito fatorial com coeficiente +1 .

b) Combinações com sinal negativo

Conströi-se uma tabela de dupla entrada, co locando-se na horizontal bäsica, as combinações pares dos fatores do efeito fatorial a ser confundido, lembrando que (1), testemunha, è a combinação dos fatores zero a zero. A vertical bäsica tem a mesma constituição da tabela para o caso anterior.

Supondo que se queira confundir a intera ção $A B D$ do fatorial $2^{5}$, determina-se primeiramente as combi nações pares, impares e restantes, ou sejam: 
combinações pares: (1), ab, ad, bd combinações impares: $a, b, d$, abd combinações restantes: (1), c, e, ce Uma vez obtidas as combinações, confecciona- se as tabelas de acordo com o que foi dito anteriormente, ou sejam:

\begin{tabular}{|c|c|c|c|c|}
\hline$A B D^{+}$ & $a$ & $b$ & $d$ & $a b d$ \\
\hline$(1)$ & $a$ & $b$ & $d$ & $a b d$ \\
\hline$c$ & $a c$ & $b c$ & $c d$ & $a b c d$ \\
\hline$e$ & $a e$ & $b e$ & $d e$ & $a b d e$ \\
\hline$c e$ & $a c e$ & $b c e$ & $c d e$ & $a b c d e$ \\
\hline
\end{tabular}

\begin{tabular}{|c|c|c|c|c|}
\hline$A B D^{-}$ & $(1)$ & $a b$ & $a d$ & $b d$ \\
\hline$(1)$ & $(1)$ & $a b$ & $a d$ & $b d$ \\
\hline$c$ & $c$ & $a b c$ & $a c d$ & $b c d$ \\
\hline$e$ & $e$ & $a b e$ & $a d e$ & $b d e$ \\
\hline$c e$ & $c e$ & $a b c e$ & acde & $b c d e$ \\
\hline
\end{tabular}

4.1.2.2. Interações com número par de fatores

a) Combinações com sinal positivo

Analogamente ao caso anterior, forma-se uma' tabela de dupla entrada, recebendo a horizontal bäsica, todas as combinações pares do efeito fatorial e na vertical básica, as combinações restantes, incluindo sempre a testemunha.

b) Combinações com sinal negativo

Neste caso, a tabela tem a horizontal bäsica constituida de combinações impares e a vertical, as mesmas 1 combinações da tabela com sinal positivo. 
Ilustra-se a seguir a construção das tabelas básicas para o confundimento da interação $A C$ do fatorial $2^{4}$ :

combinações pares: (1), ac

combinações impares: a, c

combinações restantes: (1), b, d, bd

\begin{tabular}{|c|c|c|}
\hline$A c^{+}$ & $(1)$ & $a c$ \\
\hline$(1)$ & $(1)$ & $a c$ \\
\hline$b$ & $b$ & $a b c$ \\
\hline$d$ & $d$ & $a c d$ \\
\hline$b d$ & $b d$ & $a b c d$ \\
\hline
\end{tabular}

\begin{tabular}{|c|c|c|}
\hline$A C^{-}$ & $a$ & $c$ \\
\hline$(1)$ & $a$ & $c$ \\
\hline$b$ & $a b$ & $b c$ \\
\hline$d$ & $a d$ & $c d$ \\
\hline$b d$ & $a b d$ & $b c d$ \\
\hline
\end{tabular}

4.1.3. Formação dos blocos de cađa repetição

A distribuição dos tratamentos em cada blo co ë feita de acordo com o número de blocos em cada repetição. Assim tem-se:

a) Dois blocos por repetição

Um dos blocos recebe as combinações com sinal positivo e o outro, as com sinal negativo, as quais provêm das tabelas básicas. Tomando se como exemplo o confundimento da interação $A B C$ do fatorial $2^{3}$, as tabelas bäsicas, como se sabe, têm a seguinte estrutura: 


\begin{tabular}{|c|c|c|c|c|}
\hline$A B C^{+}$ & $a$ & $b$ & $c$ & $a b c$ \\
\hline$(1)$ & $a$ & $b$ & $c$ & $a b c$ \\
\hline
\end{tabular}

\begin{tabular}{|l|l|l|l|l|}
\hline$A B C^{-}$ & $(1)$ & $a b$ & $a c$ & $b c$ \\
\hline$(1)$ & $(1)$ & $a b$ & $a c$ & $b c$ \\
\hline
\end{tabular}

e as combinações ficam assim distribuidas:

Bloco 1: $a, b, c, a b c$

Bloco 2: (1), $a b, a c, b c$

\section{b) Quatro hlocos por repetição}

Conströi-se as tabelas bäsicas de duas das três interações a serem confundidas. O primeiro bloco è for mado pelos tratamentos comuns às duas tabelas bäsicas com sinal positivo. O segundo, pelos tratamentos comuns à tabela com sinal positivo da primeira interação e à tabela com sinal negativo da segunda. O terceiro bloco, pelos tratamen tos comuns às tabelas com sinal negativo e positivo, respec tivamente, da primeira e segunda interaçöes. Finalmente, o quarto bloco, pelos tratamentos comuns às tabelas com sinal negativo.

Seja, por exemplo, o confundimento das interaçöes $A B C, A D$ e $B C n$ do fatorial $2^{4}$, obtidas escolhendo - se $A B C$ em primeiro lugar e, posteriormente, as outras duas a partir do produto $(A+B C) D=A D+B C D$. As tabelas bási cas, como se sabe, são construidas para duas quaisquer das três interações escolhidas, ou sejam: $B C D=A D$. 


\begin{tabular}{|c|c|c|c|c|}
\hline$B C D^{+}$ & $b$ & $c$ & $d$ & $b c d$ \\
\hline$(1)$ & $b$ & $c$ & $d$ & $b c d$ \\
\hline$a$ & $a b$ & $a c$ & $a d$ & $a b c d$ \\
\hline
\end{tabular}

\begin{tabular}{|c|c|c|c|c|}
\hline $\operatorname{erc}$ & $(1)$ & $b c$ & $b d$ & $c d$ \\
\hline$(1)$ & $(1)$ & $b c$ & $b d$ & $c d$ \\
\hline$a$ & $a$ & $b f b c$ & $a b d$ & $a c d$ \\
\hline
\end{tabular}

\begin{tabular}{|c||c|c|}
\hline$A D^{+}$ & $(1)$ & $a d$ \\
\hline$(1)$ & $(1)$ & $a d$ \\
\hline$b$ & $b$ & $a b d$ \\
\hline$c$ & $c$ & $a c d$ \\
\hline$b c$ & $b c$ & $a b c d$ \\
\hline
\end{tabular}

\begin{tabular}{|c||c|c|}
\hline$A D^{-}$ & $a$ & $d$ \\
\hline$(1)$ & $a$ & $d$ \\
\hline$b$ & $a b$ & $b d$ \\
\hline$c$ & $a c$ & $c d$ \\
\hline$b c$ & $a b c$ & $b c d$ \\
\hline
\end{tabular}

De acordo com o que foi dito anteriormente os quatro blocos têm os tratamentos distribuidos da seguinte maneira:

$$
\begin{aligned}
& \text { Bloco } 1:(++): b, c, a d, \text { abcd } \\
& \text { Bloco } 2:(+-): d, b c d, a b, a c \\
& \text { Bloco } 3:(-+):(1), b c, a b d, \text { acd } \\
& \text { Bloco } 4:(--): b d, c d, a, a b c
\end{aligned}
$$

\section{c) Oito blocos nor repetição}

Constroem-se as tabelas bäsicas de três qualsquer das sete interaçöes a serem confundidas, exceto a interação utilizada para a obtenção das outras seís. Cada bloco, analogamente ao caso anterior de quatro blocos,. ë formado segundo as combinações com sinal,,,+++++-++++-- , 
,,-+++--++ e ---, das tabelas bäsicas referentes às inte rações selecionadas, onde o primeiro, segundo e terceiro si nais de cada conjunto referem-se às combinações da primei ra, segunda e terceira interações, respectivamente.

Como ilustração, mostra-se, a seguir,

o confundimento das interações $B D, C E, A B C, A B E, A C D, A D E$ e $B C D E$ do fatorial $2^{5}$, selecionadas atravës do critério mostrado' no sub-item 4.1 .1 , partindo-se de $A B C$.

As tabelas bäsicas, conforme frisou-se,são confeccionadas para três quaisquer das sete interações, exceto $A B C$. Neste exemplo, optou-se pelas interações $A B E$, $A C D$ e ADE.

\begin{tabular}{|c|c|c|c|c|}
\hline$A B E^{+}$ & $a$ & $b$ & $e$ & $a b e$ \\
\hline$(1)$ & $a$ & $b$ & $e$ & $a b e$ \\
\hline$c$ & $a c$ & $b c$ & $c e$ & $a b c e$ \\
\hline$d$ & $a d$ & $b d$ & $d e$ & abde \\
\hline$c d$ & $a c d$ & $b c d$ & $c d e$ & abcde \\
\hline
\end{tabular}

\begin{tabular}{|c|c|c|c|c|}
\hline$A \cap E^{-}$ & $(1)$ & $a b$ & $a e$ & $b e$ \\
\hline$(1)$ & $(1)$ & $a b$ & $a e$ & $b e$ \\
\hline$c$ & $c$ & $a b c$ & $a c e$ & $b c e$ \\
\hline$d$ & $d$ & $a b d$ & $a d e$ & $b d e$ \\
\hline$c d$ & $c d$ & $a b c d$ & $a c d e$ & $b c d e$ \\
\hline
\end{tabular}

\begin{tabular}{|c|c|c|c|c|}
\hline$A C D^{+}$ & $a$ & $c$ & $d$ & $a c d$ \\
\hline$(1)$ & $a$ & $c$ & $d$ & $a c d$ \\
\hline$b$ & $a b$ & $b c$ & $b d$ & $a b c d$ \\
\hline$e$ & $a e$ & $c e$ & $d e$ & $a c d e$ \\
\hline$b e$ & $a b e$ & $b c e$ & $b d e$ & $a b c d e$ \\
\hline
\end{tabular}

\begin{tabular}{|c||c|c|c|c|}
\hline$A C D^{-}$ & $(1)$ & $a c$ & $a d$ & $c d$ \\
\hline$(1)$ & $(1)$ & $a c$ & $a d$ & $c d$ \\
\hline$b$ & $b$ & $a b c$ & $a b d$ & $b c d$ \\
\hline$e$ & $e$ & $a c e$ & $a d e$ & $c d e$ \\
\hline$b e$ & $b e$ & $a b c e$ & $a b d e$ & $b c d e$ \\
\hline
\end{tabular}




\begin{tabular}{|c||c|c|c|c|}
\hline$A D E^{+}$ & $a$ & $d$ & $e$ & $a d e$ \\
\hline$(1)$ & $a$ & $d$ & $e$ & $a d e$ \\
\hline$b$ & $a b$ & $b d$ & $b e$ & $a b d e$ \\
\hline$c$ & $a c$ & $c d$ & $c e$ & $a c d e$ \\
\hline$b c$ & $a b c$ & $b c d$ & $b c e$ & $a b c d e$ \\
\hline
\end{tabular}

\begin{tabular}{|c||c|c|c|c|}
\hline$A D E$ & $(1)$ & $a d$ & $a e$ & $d e$ \\
\hline$(1)$ & $(1)$ & $a d$ & $a e$ & $d e$ \\
\hline$b$ & $b$ & $a b d$ & $a b e$ & $b d e$ \\
\hline$c$ & $c$ & $a c d$ & $a c e$ & $c d e$ \\
\hline$b c$ & $b c$ & $a b c d$ & $a b c e$ & $b c d e$ \\
\hline
\end{tabular}

De acordo com o exposto, a constituição dos bo ocos è a seguinte:

Bloco $1:(+++): a, c e, b d$, abcde

Bloco $2:(++-):$ abe, bc, de, acd

Bloco $3:(+-+): e, a c$, abde, bcd

Bloco $4:(+--): b$, abce, ad, cde

Bloco $5:(-++): a b, b c e, d$, acde

Bloco $6:(-+-):$ ae, c, bde, abcd

Bloco $7:(--+):$ be, abc, ade, cd

Bloco 8:(--) : (1), ace, abd, bcde

\subsubsection{Formação de blocos de duas unidades}

Os blocos de duas unidades, independentemente do fatorial, podem ser formados diretamente, sem o auxilio' das tabelas bäsicas. Os dois tratamentos de cada bloco devem ser tais que, em conjunto, formem todas as letras representa tivas dos fatores, sem, no entanto, repeti-las. 
Assim, por exemplo, os oito blocos de duas unidades do fatorial $2^{4}$ são:

$$
\begin{aligned}
& \text { Bloco 1: (1), abcd } \\
& \text { Bloco 2: a, bcd } \\
& \text { Bloco 3: b, acd } \\
& \text { Bloco 4: c, abd } \\
& \text { Bloco 5: d, abc } \\
& \text { Bloco 6: ab, cd } \\
& \text { Bloco 7: ac, bd } \\
& \text { Bloco 8: ad, bc }
\end{aligned}
$$

As interações que resultam confundidas são todas aquelas com número par de fatores. No exemplo citado, são: $A B, A C, A D, B C, B \cap, C D$ e $A B C D$.

\subsubsection{Construção de fatoriais balanceados}

Um fatorial è dito halanceado quando fornece a mesma informação relativa nas interações confundidas que envolvam o mesmo número de fatores.

Entende-se por informação relativa de um efeito fatorial, a razão entre a sua variância sem confundimento e a com confundimento. A informação relativa varia en tre zero e um; zero quando o efeito fatorial está completamente confundido e um quando o mesmo está livre de confundi 
mento.

Em 4.6 dä-se o processo geral de obtenção da informação relativa dos efeitos fatoriais.

A seguir serão vistas diversas opções de cons trução de fatoriais balanceados até o fatorial $2^{5}$.

\subsubsection{Fatorial $2^{2}$}

Confunde-se a interação $A B$, pelo processo des crito em 4.1.4, em todas as repetições, originando blocos de duas unidades. Para tornar possivel a anălise de variancia, deve-se usar duas ou mais repetições. O plano é dado em ' 5.1.1. de Resultados.

$4.1 \cdot 5 \cdot 2$. Fatorial $2^{3}$

a) Blocos de quatro unidades

A interação de mais alta ordem, $A B C$, pode ser confundida em todas as repetições. Entretanto, caso haja interesse em analisar esta interação, poderão ser formadas 3 repetições, confundindo-se em cada uma delas as interações ' $A B$. $A C$ e $B C$, obtendo-se em cada uma $2 / 3$ da informação relat $\underline{i}$ va. Ou ainda, confundindo-se $A B C$ em uma quarta repetição, re sultando conseqüentemente, uma informação relativa de $3 / 4$ pa ra todas as interações confundidas. 
b) Blocos de duas unidades

Pelo processo dado em 4.1.4, pode-se formar quatro blocos de duas unidades. As interações confundidas' são as de dois fatores ou sejam: $A B, A C$ e $B C$.

os planos com blocos de quatro e de duas unidades são dados em 5.1.2. de Resultados.

\subsubsection{Fatorial $2^{4}$}

a) Blocos de oito unidades

Confunde-se ACCD em todas as repetições.

b) Blocos de quatro unidades

Neste caso, confunde-se interações duplas e triplas. O balanceamento è obtido confundindo-se em cada uma das repetições uma das interações duplas e duas das triplas, conforme se segue:

1a. repetição: $(A+B C) D$ : $A D, B C D, A B C$

2a. repetição: $(B+A C) D: B D, A C D, A B C$

3a. repetição: $(C+A B) \cap: C D, A B D, A B C$

4a. repetição: $(A+B D) C: A C, B C D, A C D$

5a. repetição: $(\cap+A D) C: B C, A C D, A B D$

6a. repetição: $(A+C D) B: A B, B C D, A C D$ 
Nesse conjunto balanceado, obtëm-se $5 / 6$ da informação relativa nas interações de primeira ordem e 1/2, nas interações triplas.

c) Blocos de duas unidades

$$
\text { Pelo processo descrito em } 4.1 .4 \text {. re- }
$$
sultam oito blocos de duas unidades. As interações que ficam confundidas são: $A C, A C, A \cap, B C, B \cap, C \cap$ e $A B C D$.

Os conjuntos balanceados em blocos de cito, de quatro e de duas unidades são dados em 5.1 .3 de Resultados.

$$
\text { 4.1.5.4. Fatorial } 2^{5}
$$

a) Blocos de dezesseis unidades

A interação ABCOE pode ser confundida em todas as repetições.

\section{b) Rlocos de oito unidades}

Confundem-se as interações de três e de quatro fatores. Dentre os diversos conjuntos balanceados que podem ser formados um è dado a seguir, onde em cada repetição se confundem duas interações triplas e uma quädrupla. 
1a. repetição: $(A B+C \cap) E: A B E, C D E, A D C D$

2a. repetição: $(A C+B E)$ D ACก, BDE, ABCE

3a. repetição: $(A E+D \cap) C: A C E, B C D, A B D E$

4a. repetição: $(A D+C E) B: A B D, B C E, A C D E$

5a. repetição: $(B C+\cap E) A: A B C$, ADE, חCDE

Cada interação fornece $4 / 5$ da informação re-

lativa.

c) Blocos de quatro unidades

Forma-se um conjunto balanceado com cinco re petições confundindo-se, em cada uma, duas interações duplas, três triplas e uma de ouatro fatorks. Dessa forma, ob tèm-se 4/5 da informação relativa nas interações de dois e de quatro fatores. Nas interações triplas a informação rela tiva $\bar{e} 3 / 5$.

Dentre as diversas opções, um dos conjuntos' balanceados è o seguinte:

la.repetição: $(A+B C) D,(B+A C) E,(C+A B) D E: A \cap, B E, A B C$, ACE, PCD, CDE, ACDE.

2a.repetição: $(A+B n) C,(D+A B) E,(B+A D) C F: A C, D E, A B D$, $B C E, A D E, B C D, A C D E$.

3a.repetição: $(E+A B) C,(B+A E) D,(A+B E) C D: B D, C E, A B C$, $A B E, A C \cap, A D E, R C D E$.

4a.repetição: $(A+C E) B,(C+A E) D,(E+A C) B \cap: A B, C \cap, A C E$, 
ADE, BCE, BDE, ABCD

5a.repetição: $(E+B D) A,(B+D E) C,(\cap+B E) A C: A E, B C, A B D$, ACN, BDE, CDE, ABCE

d) Blocos de duas unidares

Os dezesseis blocos de duas unidades são obtidos pelo processo dado em 4.1.4. As interações que resultam confundidas são: $A B, A C, A \cap, A E, B C, B \cap, D E, C D, C E, D E$, $A B C D, A B C E, A B \cap E, A C \cap E$ e RCDE.

os conjuntos balanceados em blocos de dezesseis, de oito, de quatro e de duas unidades encontram-se em 5.1.4. de Resultados.

4.1.6. Propriedades das tabelas básicas

As duas propriedades seguintes serão utiliza das para o confundimento de fatoriais quando todos os fatores, ou pelo menos um, possuam mais de dois níveis.

Propriedade 1: Considere-se o confundimento de um dos efeitos principais do fatorial $2^{3}$, como por iexemplo, o efeito $A$ cujas tabelas bàsicas são: 

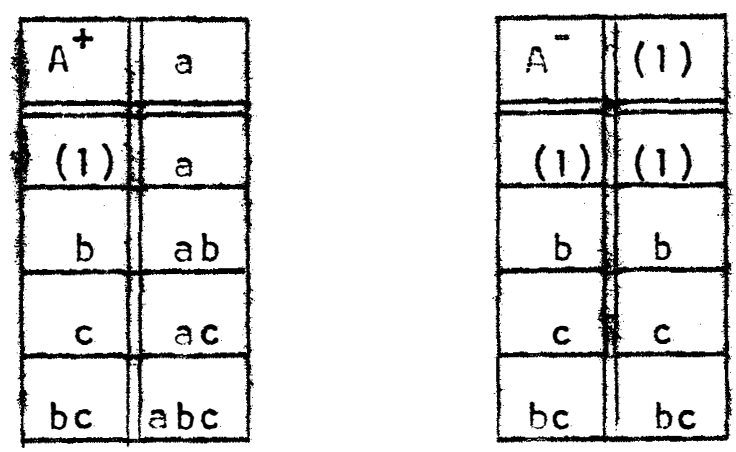

A partir dessas tabelas, forma-se um arranjo na forma matricial com as combinações das verticais bäsicas de tal modo que a primeira linha tenha como elementos a com binação (1) de cada tahela e que a segunda linha seja com posta de qualquer das combinações restantes, porém nunca de quelas com nümero de fatores superior a um. Assim procedendo, os arranjos matriclais possiveis de serem formados, nes te caso, são:

$$
\left(\begin{array}{ll}
(1)^{+} & (1)^{-} \\
b^{+} & b^{-}
\end{array}\right] \text {e }\left(\begin{array}{ll}
(1)^{+} & (1)^{-} \\
c^{+} & c^{-}
\end{array}\right)
$$

Os elementos desses arranjos poderão ser substituidos pelas combinações dos fatores, das tabelas bäsicas, a eles associadas, ou seja:
$a_{a b}^{a}$
(1)
e
$\int_{a c}^{a}$
(1) 
Considerando-se o primeiro arranjo matricial e tomando-se as diagonais principal e secundäria, verifica- se que as mesmas correspondem às horizontais bäsicas das tabelas estruturadas para o confundimento de $A B$. A diagonal principal corresponde à horizontal bäsica da tabela com sinal negativo e a secundāria à horizontal da tabela bäsica' com sinal positivo, ou seja:

\begin{tabular}{|c|c|c|}
\hline$A B^{+}$ & $(1)$ & $a b$ \\
\hline$(1)$ & $(1)$ & $a b$ \\
\hline$c$ & $c$ & $a b c$ \\
\hline
\end{tabular}

\begin{tabular}{|c|c|c|}
\hline$A B^{-}$ & $a$ & $b$ \\
\hline$(1)$ & $a$ & $b$ \\
\hline$c$ & $a c$ & $b c$ \\
\hline
\end{tabular}

Verifica-se, pelo exposto, que as tahelas do confundimento de A constituem o ponto de partida para o con fundimento de uma interação de ordem imediatamente superior, no caso, da interação Ar.

Agora, a partir das tabelas bäsicas do confundimento de $A B$, pode-se construir as tabelis bäsicas do confundimento de $A B C$, seguindo a mesma linha de raciocínio, formando-se o seguinte arranjo matricial:

$$
\left(\begin{array}{ll}
(1)^{+} & (1)^{-} \\
c^{+} & c^{-}
\end{array}\right)
$$


cujos elementos serão substituidos pelas combinações, das ta belas básicas, a eles associadas, resultando:

$$
\left[\begin{array}{ll}
(1), a b & a, b \\
c, a b c & a c, b c
\end{array}\right]
$$

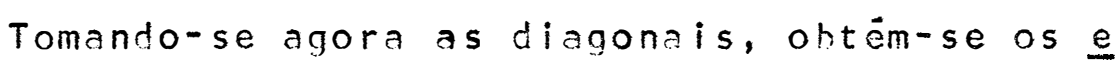
lementos integrantes das horizontais das tabelas básicas ' correspondentes ao confundimento da interação $A B C$, ou sejam:

$$
\begin{aligned}
& \text { Diagonal principal:(-): (1), ab, } a c, b c \\
& \text { Diagonal secundäria: }(+): a, b, c, a b c
\end{aligned}
$$

Propriedade 2: A partir da vertical hásica das tabelas bási cas de $y$ efeito fatorial qualquer, de um fatorial $2^{n-1}$, Po de-se estruturar a vertical häsica das tahelas do mesmo efeito, no fatorial $2^{n}$, desde que se acrescente às combina ções jä existentes, outras novas, formadas pelos seus prodü tos com o nivel superior to fator restante.

Considere-se, por exemplo, as tabelas bäsi cas de confundimento do fator $A$, em um fatorial $2^{2}$ :

\begin{tabular}{|c|c|}
\hline$A^{+}$ & $a$ \\
\hline$(1)$ & $a$ \\
\hline$b$ & $a b$ \\
\hline
\end{tabular}

\begin{tabular}{|l|l|}
\hline$A^{-}$ & $(1)$ \\
\hline$(1)$ & $(1)$ \\
\hline$b$ & $b$ \\
\hline
\end{tabular}


Para se obter as tabelas básicas de confund mento do fator $A$, no fatorial $2^{3}$, basta acrescentar à verti cal băsica do fatorial $2^{2}$ o produto das combinações jä exis tentes pelo nivel superior do fator $C$, ou seja $\underline{c}$.

Portanto,

\begin{tabular}{|c|c|}
\hline$A+$ & $a$ \\
\hline$(1)$ & $a$ \\
\hline$b$ & $a b$ \\
\hline$c$ & $a c$ \\
\hline$b c$ & $a b c$ \\
\hline
\end{tabular}

\begin{tabular}{|c|c|}
\hline$A^{-}$ & $(1)$ \\
\hline$(1)$ & $(1)$ \\
\hline$b$ & $b$ \\
\hline$c$ & $c$ \\
\hline$b c$ & $b c$ \\
\hline
\end{tabular}

A seguir, multiplicando cada combinação da vertical básica pelo segundo nível do fator $D$, obtêm-se novas combinações que, juntamente com as existentes, formarão a vertical bäsica das tabelas bäsicas do confundimento do fator A no fatorial $2^{4}$, ou sejam:

\begin{tabular}{|c|c|}
\hline$A+$ & $a$ \\
\hline$(1)$ & $a$ \\
\hline$b$ & $a b$ \\
\hline$c$ & $a c$ \\
\hline$b c$ & $a b c$ \\
\hline$d$ & $a d$ \\
\hline$b d$ & $a b d$ \\
\hline$c d$ & $a c d$ \\
\hline$b c d$ & $a b c d$ \\
\hline
\end{tabular}

\begin{tabular}{|c||c|}
\hline$A$ & $(1)$ \\
\hline$(1)$ & $(1)$ \\
\hline$b$ & $b$ \\
\hline$c$ & $c$ \\
\hline$b c$ & $b c$ \\
\hline$d$ & $d$ \\
\hline$b d$ & $b d$ \\
\hline$c d$ & $c d$ \\
\hline$b c d$ & $b c d$ \\
\hline
\end{tabular}


4.2. Confundimento da série $3^{\text {n }}$

Nestes fatoriais, como cada fator possui três niveis, estes serão representados pelos algarismos 0,1 e 2 . Nas tabelas bảsicas, uma cēlula proveniente da combinação ' 010 com 1n2, por exemplo, será 112 , isto é, será a soma algëbrica das duas combinações. Fm particular, a combinação ' 000 corresponderä ao tratamento (1) da série $2^{n}$. Tambëm não serão mais usados os sinais + e -, nas tahelas bäsicas, mas os algarismos em potência $n, 1,2$, etc; cuja finalidade serä apenas para a distinção das tabelas häsicas.

o confundimento é feito em função das proprie dades dadas em 4.1.6.

\subsubsection{Fatorial. $3^{2}$}

Considere-se, a título de ilustração, a estrü turação do confundimento da interação $A$. Parte-se das tabelas bäsicas do confundimento do efeito $A$, onde as horizontais bäsicas são formadas por qualquer um dos três niveis de A, independentemente de ordem. Assim tem-se: 


\begin{tabular}{|l|l|}
\hline$A^{0}$ & 20 \\
\hline 00 & 20 \\
\hline 01 & 21 \\
\hline 02 & 22 \\
\hline
\end{tabular}

\begin{tabular}{|c|c|}
\hline$A^{3}$ & 10 \\
\hline 00 & 10 \\
\hline 01 & 11 \\
\hline 02 & 12 \\
\hline
\end{tabular}

\begin{tabular}{|l|l|}
\hline$A^{2}$ & 00 \\
\hline 00 & 00 \\
\hline 01 & 01 \\
\hline 02 & 02 \\
\hline
\end{tabular}

Para cada combinaçäo da vertical bäsica de $A^{0}$, faz-se corresponder duas combinações das verticaís bäsi cas de $A^{1}$ e de $A^{2}$, formando-se o seguinte arranjo matrici a):

$$
\begin{aligned}
& M_{0}:\left\{(00)^{0}\right\}\left\{\begin{array}{ll}
(01)^{2} & (01)^{2} \\
(02)^{2} & (02)^{2}
\end{array}\right\} \\
& M_{1}=\left[(01)^{0}\right]\left[\begin{array}{ll}
(02)^{2} & (02)^{2} \\
(00)^{1} & (00)^{2}
\end{array}\right] \\
& M_{2}=\left[(02)^{0}\right]\left[\begin{array}{ll}
(00)^{2} & (00)^{2} \\
(01)^{2} & (01)^{2}
\end{array}\right]
\end{aligned}
$$

Observe-se que, a segunda ou ültima linha de cada arranjo deverá ser a primeira do sequinte.

Substituindo-se os elementos desses arranjos pelas combinações correspondentes das tahelas häsicas, a eles associados, obtëm-se: 


$$
\begin{aligned}
& M_{0}:\left[\begin{array}{l}
20 \\
1
\end{array}\right)\left\{\begin{array}{ll}
11 & 01 \\
12 & 02
\end{array}\right\} \\
& M_{1}:\left\{\begin{array}{ll}
21 & 1
\end{array}\right]\left(\begin{array}{ll}
12 & 02 \\
10 & 00
\end{array}\right) \\
& M_{2}:\left[\begin{array}{l}
22 \\
10
\end{array}\right]\left(\begin{array}{ll}
10 & 00 \\
11 & 01
\end{array}\right\}
\end{aligned}
$$

Este conjunto gera duas repetições, cada uma com três blocos de três unidades. os blocos da primeira são formados pelos tratamentos das diagonais principais de Mo. $M_{1}$ e $M_{2}$ acrescidos da combinação em separado, ou seja:

$$
\begin{aligned}
& \text { Bloco 1: 20, 11, } 02 \\
& \text { Bloco 2: 21, 12, } 00 \\
& \text { Bloco 3: 22, 10, } 01
\end{aligned}
$$

A segunda repetição resulta das diagonais se cundärias de $M_{a}, M_{1}$ e $M_{2}$ tambēm acrescidas da combinação em separado.

O conjunto balanceado, composto pelas duas repetições, ë dado em 5.2.1 de Resultados e fornece 1/2 da informação relativa na interação $A B$.

o mesmo conjunto balanceado será obtido partindo-se das tabelas básicas do confundimento do fator $B$. De modo semelhante ao caso anterior constroem-se as tabelas 
desse fator, dispondo-se nas horizontais básicas de cada ta bela um dos niveis de $B$, independentemente de ordem. Assim, por exemplo, tem-se:

\begin{tabular}{|c||c|}
\hline $8^{0}$ & 01 \\
\hline 00 & 01 \\
\hline 10 & 11 \\
\hline 20 & 21 \\
\hline
\end{tabular}

\begin{tabular}{|c||c|}
\hline$B^{2}$ & 00 \\
\hline 00 & 00 \\
\hline 10 & 10 \\
\hline 20 & 20 \\
\hline
\end{tabular}

\begin{tabular}{|c||c|}
\hline $3^{2}$ & 02 \\
\hline 00 & 02 \\
\hline 10 & 12 \\
\hline 20 & 22 \\
\hline
\end{tabular}

$$
\begin{aligned}
& M_{0}=\left\{(00)^{0}\right]\left[\begin{array}{ll}
(10)^{2} & (15)^{2} \\
(20)^{!} & (20)^{2}
\end{array}\right] \\
& M_{1}:\left[(10)^{0}\right]\left[\begin{array}{ll}
(20)^{3} & (20)^{2} \\
(00)^{1} & (00)^{2}
\end{array}\right] \\
& M_{2}:\left[(20)^{0}\right]\left[\begin{array}{ll}
(00)^{1} & (00)^{2} \\
(10)^{2} & (10)^{2}
\end{array}\right]
\end{aligned}
$$

Substituindo-se os elementos dessas matrizes pelas combinações das tabelas băsicas correspondentes, a eles associados, tem-se:

$$
M_{0}:(01)\left[\begin{array}{ll}
10 & 12 \\
20 & 22
\end{array}\right]
$$




$$
\begin{aligned}
& M_{1}:[11]\left[\begin{array}{ll}
20 & 22 \\
00 & 02
\end{array}\right] \\
& M_{2}=[21]\left[\begin{array}{ll}
00 & 02 \\
10 & 12
\end{array}\right]
\end{aligned}
$$

As diagonais principais de $M_{0}, M_{1}$ e $M_{2}$, acrescidas do elemento em separado, fornecem a primeira repe tição e as secundärias, a segunda.

\subsubsection{Fatorial $3^{3}$}

Neste fatorial pode-se formar repetições de três blocos de nove unidades e nove blocos de três unidades cada.

a) Blocos de nove unidades

Pela propriedade 2, citada em 4.1.6, pode-se obter a interação $A B$, confundida, do fatorial $3^{3}$, a partir' do fatorial $3^{2}$, bastando acrescentar nas verticais básicas' de cada tabela os niveis do fator C. Assim, tomando-se os' tratamentos da repetição 1, dada em 5.2.1, cada bloco gerará uma horizontal das tabelas básicas do confundimento da 
mesma interação no fatorial $3^{3}$, ou seja:

\begin{tabular}{|c|c|c|c|}
\hline$A B^{\circ}$ & 200 & 110 & 020 \\
\hline 000 & 200 & 110 & 020 \\
\hline 001 & 201 & 111 & 021 \\
\hline 002 & 202 & 112 & 022 \\
\hline
\end{tabular}

\begin{tabular}{|l|l|l|l|}
\hline$A B^{1}$ & 210 & 120 & 000 \\
\hline 000 & 210 & 120 & 000 \\
\hline 001 & 211 & 121 & 001 \\
\hline 002 & 212 & 122 & 002 \\
\hline
\end{tabular}

\begin{tabular}{|c|c|c|c|}
\hline$A \sigma^{2}$ & 220 & 100 & 010 \\
\hline 000 & 220 & 100 & 010 \\
\hline 001 & 221 & 101 & 011 \\
\hline 002 & 222 & 102 & 012 \\
\hline
\end{tabular}

Repetindo-se o processo dade em 4.2.1, formam-se os arranjos matriclais:

$$
M_{0}:\left[(0.00)^{0}\right]\left[\begin{array}{ll}
(001)^{1} & (001)^{2} \\
(0.02)^{1} & (0.02)^{2}
\end{array}\right]
$$




$$
\begin{aligned}
& M_{1}=\left\{(001)^{0}\right)\left[\begin{array}{ll}
(002)^{1} & (002)^{2} \\
(000)^{1} & (000)^{2}
\end{array}\right] \\
& M_{2}=\left\{(002)^{-0}\left[\begin{array}{ll}
(000)^{1} & (000)^{2} \\
(001)^{2} & (001)^{2}
\end{array}\right]\right.
\end{aligned}
$$

Inserindo os tratamentos correspondentes a cada elemento desses arranjos, das tabelas básicas, obtém -se:

$M_{0}=(200,110,020)\left[\begin{array}{ll}211,121,001 & 221,101,011 \\ 212,122,002 & 222,102,012\end{array}\right]$ $\left.M_{1}=[201,111,021]\right\}\left[\begin{array}{lll}212,122,002 & 222,102,012 \\ 210,120,000 & 220,100,010\end{array}\right]$ $M_{2}=[202,112,022]\left[\begin{array}{lll}210,120,000 & 220,100,010 \\ 211,121,001 & 221,101,011\end{array}\right]$

As diagonais principais de $M_{0}, M_{1}$ e $M_{2}$, formam dos tratamentos dos blocos da primeira repetição, ou se jam: 
B1oco 1: 200, 110, 020, 211, 121, 001, 222, 102, 012

B10co 2: 201, 111, 021, 212, 122, 002, 220, 100, 010

Bloco 3: 202, 112, 022, 210, 120,000, 221, 101, 011

As diagonais secundärias desses três arran jos fornecem os tratamentos dos blocos da segunda repetição. o fatorial balanceado consta de quatro repetições, sendo a terceira e a quarta obtidas através da apli cação do mesmo processo na repetição 11 , do confundimento de $A B$, do fatorial $3^{2}$.

A interação $A B C$ fica parcialmente confundida em cada uma das quatro repetições e o conjunto balanceado' fornece $3 / 4$ da informação relativa nesta interação.

b) Blocos de três uniriades

Cada tabela bäsica do confundimento de $A B$, do item a), pode ser considerada como três tabelas bäsicas de um efeito principal, ou sejam:

De $A B^{0}:$\begin{tabular}{|l|l|}
\hline$A B^{00}$ & 200 \\
\hline 000 & 200 \\
\hline 001 & 201 \\
\hline 002 & 202 \\
\hline
\end{tabular}

\begin{tabular}{|c|c|}
\hline$A 001$ & 110 \\
\hline 000 & 110 \\
\hline 001 & 111 \\
\hline 002 & 112 \\
\hline
\end{tabular}

\begin{tabular}{|c||c|}
\hline$A B^{02}$ & 020 \\
\hline 000 & 020 \\
\hline 001 & 021 \\
\hline 002 & 022 \\
\hline
\end{tabular}


De $A B^{2}$ :

\begin{tabular}{|c|c|}
\hline$A B^{10}$ & 210 \\
\hline 000 & 210 \\
\hline 001 & 211 \\
\hline 002 & 212 \\
\hline
\end{tabular}

\begin{tabular}{|c|c|}
\hline$A B^{11}$ & 120 \\
\hline 000 & 120 \\
\hline 001 & 121 \\
\hline 002 & 122 \\
\hline
\end{tabular}

\begin{tabular}{|l||l|}
\hline$A R^{2}$ & 000 \\
\hline 000 & 000 \\
\hline 001 & 001 \\
\hline 002 & 002 \\
\hline
\end{tabular}

De $A B^{2}$ :

\begin{tabular}{|c|c|}
\hline$A B^{20}$ & 220 \\
\hline 000 & 220 \\
\hline 001 & 221 \\
\hline 002 & 222 \\
\hline
\end{tabular}

\begin{tabular}{|c||c|}
\hline$A B^{22}$ & 010 \\
\hline 000 & 010 \\
\hline 001 & 011 \\
\hline 002 & 012 \\
\hline
\end{tabular}

Reaplicando-se o processo descrito em 4.2.1, pode-se escrever as matrizes:

$\left.M_{00}=\left[\begin{array}{ll}20 & 0\end{array}\right]\left[\begin{array}{ll}111 & 021 \\ 112 & 022\end{array}\right] \quad M_{01}:[201]\left[\begin{array}{ll}112 & 022 \\ 110 & 020\end{array}\right] \quad M_{02}:(202]\right)\left[\begin{array}{ll}110 & 020 \\ 111 & 021\end{array}\right]$

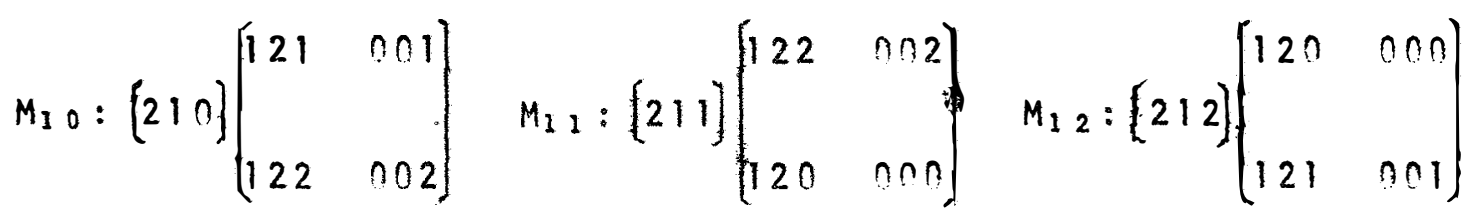
$\left.M_{20}=[220]\left[\begin{array}{ll}101 & 011 \\ 102 & 012\end{array}\right] \quad M_{22}:[221]\right)\left[\begin{array}{ll}102 & 012 \\ 10 n & 010\end{array}\right] \quad M_{22}:\{222]\left[\begin{array}{ll}100 & 010 \\ 101 & 011\end{array}\right]$

Cada diagonal, acrescida da combinação inicl al, corresponderá a um dos nove blocos da primeira repeti ção. Assim, tem-se: 
Bloco 1: 203, 111, 022

B10co 2: 201, 112,020

B10co 3: 2n2, 110, 021

Bloco 4: $21 \cap, 121,002$

B1000 5: 211, 122, 000

B10co 6: 212, 120, 001

B10co 7. $220,1 \% !, 012$

B10co 8: $221,102,010$

Bloco 9: 222, 10n, 011

Da mesma forma, obtēm-se a segunda repetição operando-se com as diagonais secundárias.

o conjunto balanceado é composto de quatro' repetições, sendo que a terceira e a quarta são obtidas de maneira análoga, a partir das tabelas básicas da segunda re petição do confundimento de $A \cap$ do fatorial $3^{2}$.

Em cada repetição desse conjunto resultam ' parcialmente confundidas as interações $A \cap, A C, B C$ e $A \cap C$.

0 conjunto balanceado fornece $1 / 2$ da informa ção relativa nas interações duplas e $3 / 4$ em $A B C$.

Em 5.2.2. de Resultados, encontram - se os conjuntos balanceados em blocos de nove e de três unidades. 
4.2.3. Fatorial $3^{4}$

Serä vista a formação dos conjuntos balancea dos em blocos de vinte e sete, de nove e de trîs uniuades.

a) Blocos de vinte e sete unidades

Tomando-se as quatro repetições do fatorial' $3^{3}$ em blocos de nove unidades, dadas em 5.2.2, confecciona- se, para cada uma, as tabelas bäsicas da interação $A B C$, pa ra o fatorial $3^{4}$. Aplicando-se o processo de confundimento' da interação imediatamente superior (propriedade 1 , das tabelas bäsicas), obtëm-se o fatorial balanceado com oito repetlções que fornece $7 / 8$ da informação relativa na intera ção. ABCD.

A titulo de ilustração, apresentam-se as três tabelas básicas provenientes da utilização da primeira repetição do fatorial $3^{3}$ em blocos de nove unidades, ou sejam:

\begin{tabular}{|l|l|l|l|l|l|l|l|l|l|}
\hline$A 3 C^{\circ}$ & 2000 & 1100 & 0200 & 2110 & 1210 & 0010 & 2220 & 1020 & 0120 \\
\hline 0000 & 2000 & 1100 & 0200 & 2110 & 1210 & 0010 & 2220 & 1020 & 0120 \\
\hline 0001 & 2001 & 1101 & 0201 & 2111 & 1211 & 0011 & 2221 & 1021 & 0121 \\
\hline 0002 & 2002 & 1102 & 0202 & 2112 & 1212 & 0012 & 2222 & 1022 & 0122 \\
\hline
\end{tabular}




\begin{tabular}{|l|l|l|l|l|l|l|l|l|l|}
\hline$A B C^{2}$ & 2010 & 1110 & 0210 & 2120 & 1220 & 0020 & 2200 & 1000 & 0100 \\
\hline 0000 & 2010 & 1110 & 0210 & 2120 & 1220 & 0020 & 2200 & 1000 & 0100 \\
\hline 0001 & 2011 & 1111 & 0211 & 2121 & 1221 & 0021 & 2201 & 1001 & 0101 \\
\hline 0002 & 2012 & 1112 & 0212 & 2122 & 1222 & 0022 & 2202 & 1002 & 0102 \\
\hline
\end{tabular}

\begin{tabular}{|l|l|l|l|l|l|l|l|l|l|}
\hline$A B C^{2}$ & 2020 & 1120 & 0220 & 2100 & 1200 & 0000 & 2210 & 1010 & 0110 \\
\hline 0000 & 2020 & 1120 & 0220 & 2100 & 1200 & 0000 & 2210 & 1010 & 0110 \\
\hline 0001 & 2021 & 1121 & 0221 & 2101 & 1201 & 0001 & 2211 & 1011 & 0111 \\
\hline 0002 & 2022 & 1122 & 0222 & 2102 & 1202 & 0002 & 2212 & 1012 & 0112 \\
\hline
\end{tabular}

A partir destas tabelas formam-se os arran jos matriciais:

$$
\begin{aligned}
& M_{0}=\left[(0000)^{0}\right]\left[\begin{array}{ll}
(0001)^{1} & (0001)^{2} \\
(0002)^{1} & (0002)^{2}
\end{array}\right] \\
& M_{1}=\left[(0001)^{0}\right]\left[\begin{array}{ll}
(0002)^{1} & (0002)^{2} \\
(0000)^{1} & (0000)^{2}
\end{array}\right] \\
& M_{2}=\left[(0002)^{0}\right]\left[\begin{array}{ll}
(0000)^{2} & (0000)^{2} \\
(0001)^{1} & (0001)^{2}
\end{array}\right]
\end{aligned}
$$


As diagonais principais das matrizes acima, formam os três blocos da primeira repetição:

$$
\begin{aligned}
& \text { Bloco 1: }\left\{(0000)^{0} ;(0001)^{1} ;(0002)^{2}\right\} \\
& \text { Bloco 2: }\left\{(0001)^{0} ;(0002)^{1} ;(0000)^{2}\right\} \\
& \text { Bloco 3: }\left\{(0002)^{0} ;(0.00)^{1} ;(0001)^{2}\right\}
\end{aligned}
$$

substituindo na estruturação final dos blo cos, cada notação pelas suas respectivas combinaçöes inte grantes nas tabelas bäsicas, obtêm=se os 27 tratamentos' de cada bloco; assim, por exemplo, a notação $(0000)^{\circ}$ inte grante do bloco 1 , contribuirä para a sua formaçãr, com os seguintes tratamentos:

$(0000)^{\circ}: 2000,1100,0200,2110,1210,0010,2200,1020 \mathrm{e}$ 0120

A segunda repetição è obtida atravès das dia gonais secundärias desses arranjos matriciais.

Repetindo-se o processo com as tabelas bäsicas das outras três repetições do fatorial $3^{3}$, obtém-se o fatorial balanceado.

b) Blocos de nove unidades

Eosivel construir dois conjuntos balan ceados, cada conjunto composto de quatro repetições, confun dindo-se apenas as interações de três fatores. Para tanto, deve-se ter em mente que os nove niveis provenientes de to- 
das as combinações dos fatores, dois a dois, devem figurar em cada bloco de cada repetição, para evitar o confundimento de interações duplas.

As tabelas básicas que darão origem às combi nações de cada bloco são formadas a partir dos blocos de três unidades do fatorial $3^{3}$.

Como se pode verificar no sub-item 4.2.2, ca da tabela bäsica de $A B$, cujas horizontais foram tomadas da repetição 1 do fatorial $3^{2}$, foi subdividida em três e a par tir destas, formados os nove arranjos matriciais:

$\begin{array}{lll}M 00 & M_{01} & M_{02} \\ M_{10} & M_{11} & M_{12} \\ M_{20} & M_{21} & M_{22}\end{array}$

$0 s$ indices de $M$, sugerem um fatorial $3^{2}$, don de podem ser formadas duas "repetições", de acordo com os tratamentos daquele fatorial, ou seja:

$\begin{array}{ccc} & \text { "Repetição } 1 " \\ \text { "Bloco } 1 " & \text { "Rloco 2" } & \text { "Bloco } 3 " \\ M 00 & M_{10} & M_{20} \\ M_{11} & M_{21} & M_{01} \\ M_{22} & M_{02} & M_{12}\end{array}$




$\begin{array}{ccc} & \text { "Repetição } 11 " & \\ \text { "Bloco 1" } & \text { "Bloco 2" } & \text { "Bloco 3" } \\ M_{21} & M_{10} & M_{20} \\ M_{12} & M_{01} & M_{11} \\ M_{22} & M_{02}\end{array}$

E interessante lembrar que, cada elemento das "repetições l e ll", è por si um arranjo matricial. Assim por exemplo, o elemento Moo corresponde a seguinte estrutura matricial:

$$
M_{00}:[200]\left[\begin{array}{ll}
111 & 021 \\
112 & 022
\end{array}\right]
$$

As diagonais das matrizes de cada "bloco" das "repetições" acima formarão as horizontais bäsicas das tabelas que constituirão os blocos de nove unidades.

Entretanto, na "repetição l", apenas as diagonais secundärias serão utilizadas pois, conforme jä foi mencionado, as diagonais principais dessas matrizes determinam o confundimento de interações duplas. Na "repetição 11 ", pelo mesmo motivo, ut llizam-se apenas as diagonais principais. Como exemplo do confundimento de interações duplas, pode-se dispor dos elementos das diagonais principais de Moo, $M_{11}$ e $M_{22}$, constituintes do "bloco l"da "repetição l", que são: $200,111,022,211,122,000,222,100$ e 011 . Todas as 
combinações dos niveis de $A$ e $B$ e de $A$ e C encontram-se nes ses nove tratamentos; entretanto, apenas as combinações 00 , 11 e 22 de $D$ e C são encontradas, o que determinarä, posteriormente, o confundimento da interação $B C$.

llustra-se, a seguir, o processo de obtenção dos dois conjuntos balanceados em blocos de nove unidades. Com as diagonais secundärias de $M_{00}, M_{11}$ e $M_{22}$ ("bloco l", "repetição ("), constroem-se as três tabelas häsicas seguin tes:

\begin{tabular}{|c|c|c|c|}
\hline$A B^{00}$ & 2000 & 1120 & 0210 \\
\hline 0000 & 2000 & 1120 & 0210 \\
\hline 0001 & 2001 & 1121 & 0211 \\
\hline 0002 & 2002 & 1122 & 0212 \\
\hline
\end{tabular}

\begin{tabular}{|c|c|c|c|}
\hline$A B^{11}$ & 2110 & 1200 & 0020 \\
\hline 0000 & 2110 & 1200 & 0020 \\
\hline 0001 & 2111 & 1201 & 0021 \\
\hline 0002 & 2112 & 1202 & 0022 \\
\hline
\end{tabular}

\begin{tabular}{|c||c|c|c|}
\hline$A R^{22}$ & 2220 & 1010 & 0100 \\
\hline 0000 & 2220 & 1010 & 0100 \\
\hline 0001 & 2221 & 1011 & 0101 \\
\hline 0002 & 2222 & 1012 & 0102 \\
\hline
\end{tabular}


De acordo com o processo de confundimento for mam-se os seguintes arranjos matriciais:

$$
\begin{aligned}
& A_{1}:[(0000) 00]\left[\begin{array}{ll}
(0001)^{11} & (0001)^{22} \\
(0002)^{11} & (0002)^{22}
\end{array}\right] \\
& A_{2}:\left[(0001)^{0} 0\right]\left[\begin{array}{ll}
(0002)^{12} & (0002)^{22} \\
(0000)^{11} & (0000)^{22}
\end{array}\right] \\
& A_{3}:[(0002) 00]\left[\begin{array}{ll}
(0000)^{11} & (0000)^{22} \\
(0001)^{11} & (0001)^{22}
\end{array}\right]
\end{aligned}
$$

Os três primeiros blocos da primeira repetição, do primeiro conjunto balanceado, resultam das diagonais principais de $A_{1}, A_{2} \in A_{3}$, evidentemente acrescida a com binação em separado:

Aloco 1: $(0000)^{00}: 2000,1120,0210$, $(0001)^{11}: 2111,1201,0321$, $(0002)^{22}: 2222,1012,0102$.

Bloco 2: $(0001)^{00}: 2001,1121,0211$, $(0002)^{11}: 2112,1202,0022$, $(0000)^{22}: 2220,1010,0100$. 


$$
\begin{aligned}
& \text { Bloco 3: }(0002)^{00}: 2002,1122,0212, \\
&(0000)^{11}: 2110,1200,0020, \\
&(0001)^{22}: 2221,1011,0101 .
\end{aligned}
$$

Construindo-se as tabelas bäsicas com as dia gonals secundärias dos arranjos matriciais dos "blocos 2 e 3" da "repetição 1", obtêm-se os seis blocos restantes.

0 processo de obtenção da segunda repetição' é anălogo ao da primeira, desde que se crinstruam as tabelas băsicas com as diagonais principais das matrizes de cada "bloco" da "repetição 11 ".

As duas primeiras repetições do segundo conjunto balanceado são obtidas com as diagonais secundárias ' de $A_{1}, A_{2}$ e $A_{3}$. Assim, por exemplo, os três primeiros blocos da primeira repetição são os que seguem:

$$
\begin{aligned}
\text { nloco 1: } & (0000)^{00}: 200 n, 1120,0210, \\
& (0002)^{11}: 2112,12 n 2,0 n 22, \\
& (0001)^{22}: 2221,1011,0101 . \\
\text { nloco 2: } & (00 n 1)^{00}: 2001,1121,0211, \\
& (0000)^{11}: 2110,1200,0020, \\
& (0002)^{22}: 2222,1012,0102 . \\
\text { Bloco 3: } & (0002)^{00}: 2002,1122,0212, \\
& (0001)^{12}: 2111,1201,0021, \\
& (0000)^{22}: 2220,1010,010 n .
\end{aligned}
$$

Com as nove matrizes provenientes das tabe las bäsicas de $A \cap$ que formam as repetições $\| 11$ e IV do fa- 
torial $3^{3}$, em blocos de três unidades, formam-se as duas re petições restantes de cada conjunto balanceado. As nove matrizes resultantes daquelas tabelas são:

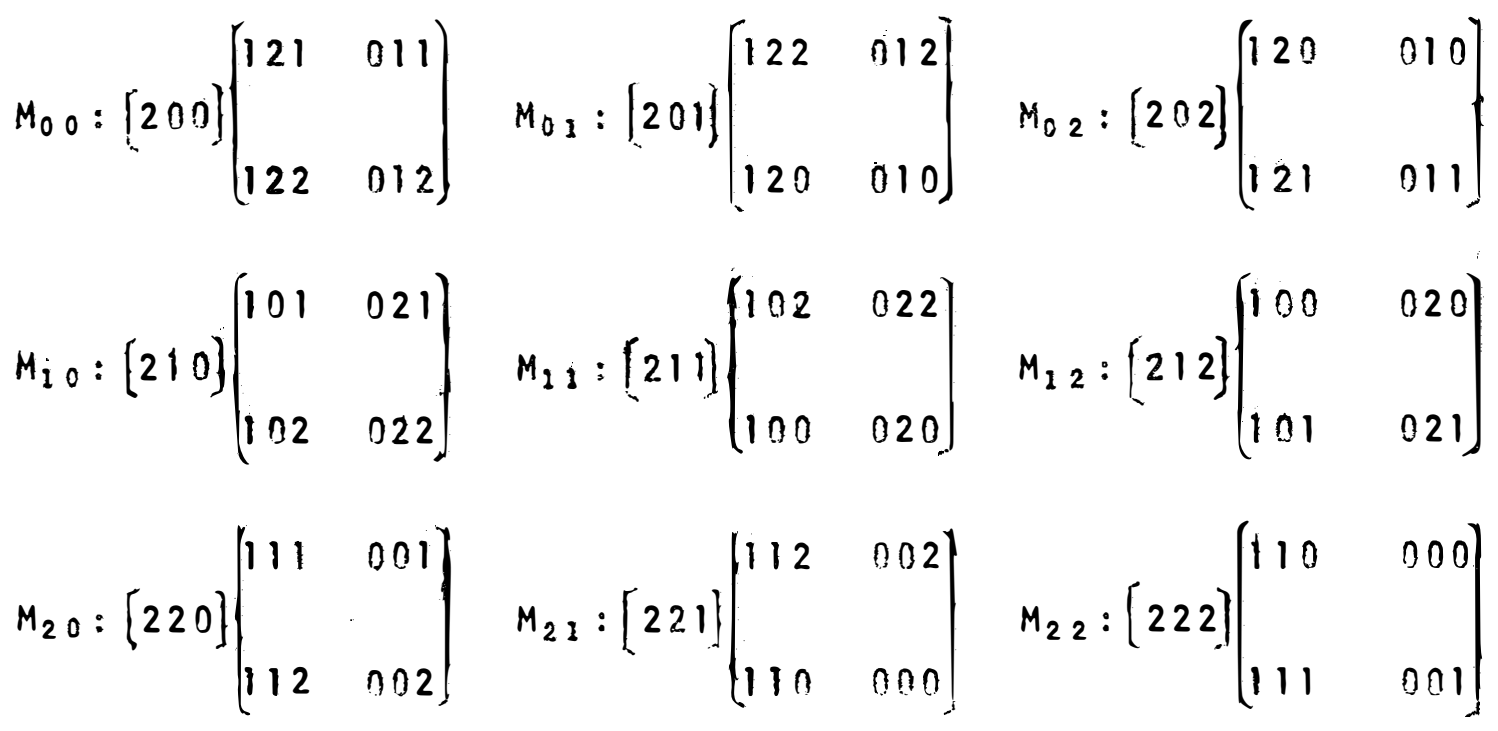

As diagonais dessas matrizes, tomadas atra vēs dos "blocos" das duas "repetiçoses" anteriormente cita das, permitirão a obtenção das repetições restantes.

A construção das tabelas bäsicas é feita atravès das diagonais principais das matrizes da "repetlção I" e das diagonais secundärias da "repetição II".

A titulo de ilustração, mostram-se as três' tabelas bäsicas formadas com as diagonais principais das matrizes $M_{0} 0, M_{11}$ e $M_{22}$ ("bloco l", "repetição l"): 


\begin{tabular}{|c||c|c|c|}
\hline$A B^{00}$ & 2000 & 1210 & 0120 \\
\hline 0000 & 2000 & 1210 & 0120 \\
\hline 0001 & 2001 & 1211 & 0121 \\
\hline 0002 & 2002 & 1212 & 0122 \\
\hline
\end{tabular}

\begin{tabular}{|c|c|c|c|}
\hline$A B^{11}$ & 2110 & 1020 & 0200 \\
\hline 0000 & 2110 & 1020 & 0200 \\
\hline 0001 & 2111 & 1021 & 0201 \\
\hline 0002 & 2112 & 1022 & 0202 \\
\hline
\end{tabular}

\begin{tabular}{|l|l|l|l|}
\hline$A B^{22}$ & 2220 & 1100 & 0010 \\
\hline 0000 & 2220 & 1100 & 0010 \\
\hline 0001 & 2221 & 1101 & 0011 \\
\hline 0002 & 2222 & 1102 & 0012 \\
\hline
\end{tabular}

Estas tabelas permitem a formação dos arranjos matriciais $A_{1}, A_{2}$ e $A_{3}$, citados anteriormente e, atra vés dos mesmos, pelas diagonais secundärias, resultam os três primeiros blocos da terceira repetição do primeiro con junto balanceado, a seguir apresentados:

Bloco 1: 200n, 121n, 012n, 2112, 1022, 0202, 2221, $1101 \mathrm{e}$ nก)

D10co 2: $2001,1211,0121,2110,1020,0200,2222,1102$ e 0012 . 
B10co 3: 2002, 1212, 0122, 2111, 1021, 0201, 2220, 1100 e nก)

Os seis blocos restantes são ohtidos de ma neira anăloga, porém utilizando os "blocos 2 e 3 " da "repetição I" $^{\prime \prime}$

A quarta repetição è obtida com o auxílio' das tabelas bäsicas formadas pelas diagonais secundärias ' das matrizes dos "blocos" da "repetição 11 ".

A terceira e quarta repetições do segundo' conjunto balanceado são obtidas trabalhando-se com as diago nais principais dos arranjos matriciais $A_{1}, A_{2}$ e $A_{3}$.

Cada conjunto balanceado fornece $3 / 4$ da in formação relativa em cada uma das interações de três fato res.

c) Blocos de três unidades

Neste caso, o balanceamento ë obtido com oito repetições, cada uma constituida de vinte e sete blocos. 0 conjunto balanceado fornece $1 / 2$ da informação relativa ' nas interaçóes de dois fatores, $3 / 4$ nas triplas e $5 / 8$ na de quatro fatores.

Para se obter esses plano, tomam-se os blocos da primeira repetição do fatorial $3^{3}$, em blocos de três unidades e com eles montam-se nove tabelas báslcas, cujas três primeiras são a seguir ilustradas: 


\begin{tabular}{|l||l|l|l|}
\hline$A 000$ & 2000 & 1110 & 0220 \\
\hline 0000 & 2000 & 1110 & 0220 \\
\hline 0001 & 2001 & 1111 & 0221 \\
\hline 0002 & 2002 & 1112 & 0222 \\
\hline
\end{tabular}

\begin{tabular}{|c|c|c|c|}
\hline$A B 01$ & 2010 & 1120 & 0200 \\
\hline 0000 & 2010 & 1120 & 0200 \\
\hline 0001 & 2011 & 1121 & 0201 \\
\hline 0002 & 2012 & 1122 & 0202 \\
\hline
\end{tabular}

\begin{tabular}{|c||c|c|c|}
\hline An02 & 2020 & 1100 & 0210 \\
\hline 0000 & 2020 & 1100 & 0210 \\
\hline 0001 & 2021 & 1101 & 0211 \\
\hline 0002 & 2022 & 1102 & 0212 \\
\hline
\end{tabular}

Cada tabela bäsica é considerada como a de um efeito principal, isto é, desdobrada em três tabelas, confor me feito em 4.2.2. Da tabela $A B^{00}$ resultam as matrizes:

$$
M_{000}:[2 n n n]\left[\begin{array}{ll}
1111 & 0221 \\
1112 & 0222
\end{array}\right)
$$




$$
M_{001}:[2001]\left[\begin{array}{ll}
1112 & 0222 \\
1110 & 0220
\end{array}\right]
$$

cujas diagonais principais formam os três primeiros blocos da primeira repetição, ou sejam:

$$
\begin{aligned}
& \text { Bloco 1: } 2 n n n, 1111,0222 \\
& \text { nloco 2: } 2001,1112,0220 \\
& \text { Bloco 3: } 2002,1110,0221 \\
& \text { ns vinte e quatro blocos resntantes dessa re }
\end{aligned}
$$
petição são obtidos de maneira anăloga, utilizando-se as ou tras oito tabelas.

$$
\text { A segunda repetição é proveniente dos elemen }
$$
tos das diagonais secundärias dos arranjos matriciais forma dos para a obtenção da primeira. Os três primeiros blocos' ธลัo:

$$
\begin{aligned}
& \text { Bloco 1: } 2000,1112,0221 \\
& \text { Bloco 2: } 20 n 1,1110,0222 \\
& \text { Ploco 3: 2nn2, } 1111,0220 \\
& \text { Por processo anälogo, btêm-se mais seis re- }
\end{aligned}
$$
petições, utilizando-se os três conjuntos de nove tabelas ' formados com as três repetições restantes do fatorial $3^{3} \mathrm{em}$ blocos de três unidades. 
As oito repetições que formam os conjuntos ba lanceados em blocos de vinte e sete e de três unidades, bom como o primeiro conjunto balanceado em blocos de nove unidades são dados em 5.2.3. de Resultados.

4.3. Confundimento da série $3 \times 2^{n}$

Para se obter o confundimento desta série, de ve-se primeiramente estruturar as tabelas básicas para o con fundimento do fatorial $3 \times 2$ e a partir deste, confundir os demais. Convém salientar que, nesta série, para não haver o confundimento de efeitos principais deve-se ter pelo menos' seis tratamentos Dor bloco. Entretanto, o processo exige a formação de blocos de três unidades, os quais servirão de ba se para a obtenção de blocos de seis tratamentos no fatorial subsequente. Assim, com blocos de três unidades do fatorial' $3 \times 2$ obtêm-se blocos de tamanho seis no $3 \times 2^{2}$ e assim su cessivamente.

\subsubsection{Fatorial $3 \times 2$}

As tabelas básicas do efeito principal $A$, com três niveis, podem ser formadas de maneira semelhante às tabelas do fatorial $2^{2}$, dispondo-se na horizontal básica da primeira, um dos niveis desse fator e na horizontal da outra 
os dois niveis restantes. Dessa forma, é possivel formar 3 pares de tabelas, cujo primeiro par è o seguinte:

\begin{tabular}{|c||c|}
\hline$A^{0}$ & 20 \\
\hline 00 & 20 \\
\hline 01 & 21 \\
\hline
\end{tabular}

\begin{tabular}{|l||l|l|}
\hline$A^{1}$ & 10 & 00 \\
\hline 00 & 10 & 00 \\
\hline 01 & 11 & 01 \\
\hline
\end{tabular}

Com as verticais bäsicas forma-se o arranjo matricial:

$$
\left[\begin{array}{ll}
(00)^{0} & (00)^{1} \\
(01)^{0} & (01)^{3}
\end{array}\right]
$$

cujos elementos das diagonais, devidamente substituidos, for marão os dois blocos da primeira repetição, os quais são:

nloco 1: $(00)^{0} ;(01)^{1}: 20,11,01$

nloco 2: $(n 1)^{0} ;(n n)^{2}: 21,10,0 n$

Os outros dois pares de tabelas são:

\begin{tabular}{|c|c|}
\hline$A^{0}$ & 10 \\
\hline $0 n$ & 10 \\
\hline 01 & 11 \\
\hline
\end{tabular}

\begin{tabular}{|l||c|c|}
\hline$A^{1}$ & $2 n$ & $0 n$ \\
\hline$n n$ & $2 n$ & $n n$ \\
\hline 01 & 21 & 01 \\
\hline
\end{tabular}

\begin{tabular}{|l|l|}
\hline$A^{0}$ & 00 \\
\hline 00 & 00 \\
\hline 01 & 01 \\
\hline
\end{tabular}

\begin{tabular}{|c||c|c|}
\hline$A^{1}$ & $2 n$ & 10 \\
\hline 00 & 20 & 2 \\
\hline$n 1$ & 21 & 11 \\
\hline
\end{tabular}


operando de maneira idêntica ao primeiro par, obtêm-se mais duas repetições. As três repetições encontram-se em 5.3.1. de Resultados, constituindo o fatorial balanceado, o qual fornece $8 / 9$ da informação relativa para o efeito principal $B$ e $5 / 9$ para a interação $A B$.

Em todas as tabelas bäsicas, deve-se dispor' os niveis do fator $A$, das horizontais básicas de $A^{\prime}$, em ordem crescente ou decrescente, para o confundimento correto' do fatorial $3 \times 2^{2}$ em blocos de três unidades.

\section{3 .2$. Fatorial. $3 \times 2^{2}$}

Neste esquema obtêm-se um conjunto balanceado em blocos de seis unidades e dois conjuntos em blocos de três.

\section{a) BIocos de seis unidades}

n esquema de blocos com seis unidades é obtido utilizando-se inicialmente os tratamentos da primeira repetição do fatorial $3 \times 2$ na confeç̧ão do par de tabelas seguinte:

\begin{tabular}{|c|c|c|c|}
\hline$A B^{\circ}$ & 200 & 110 & 010 \\
\hline 000 & 200 & 110 & 010 \\
\hline 001 & 201 & 111 & 011 \\
\hline
\end{tabular}

\begin{tabular}{|c|c|c|c|}
\hline$A B^{1}$ & 210 & 100 & 000 \\
\hline 000 & 210 & 100 & 000 \\
\hline 001 & 211 & 101 & 001 \\
\hline
\end{tabular}


A partir das verticais bäsicas dessas tabelas forma-se o arranjo matricial:

$$
\left[\begin{array}{ll}
(000)^{0} & (000)^{2} \\
(001)^{0} & (001)^{3}
\end{array}\right]
$$

- qual, através de suas diagonais, fornece as combinações' que formarão os dois blocos da primeira repetição, ou sejam: Bloco 1: $(000)^{0} ;(001)^{1}: 2 n n, 11 n, 010,211,101,001$ Bloco 2: $(001)^{0} ;(000)^{1}: 201,111,011,210,100,000$ Construindo-se as tabelas básicas com as outras duas repetições do fatorial $3 \times 2$ e repetindo-se o processo, obtêm-se as outras duas repetições, as quais, junta mente com a primeira,constituem o fatorial balanceado. Este fatorial fornece $8 / 9$ da informação relativa em BC e $5 / 9$ em $A B C$.

b) Blocos de três unidades

Cada tabela häsica construida no item a), pode ser considerada como um par de tabelas do efeito princi pal A, do fatorial $3 \times 2$. Assim considerando, pode-se construir os arranjos matriciais $M_{0}$ e $M_{1}$, dados a seguir, provenientes de $A B^{0}$ e de $A O^{1}$, respectivamente: 


$$
M_{0}:\left[\begin{array}{l}
200 \\
201
\end{array}\right]\left[\begin{array}{ll}
110 & 010 \\
111 & 011
\end{array}\right] \quad M_{1}:\left[\begin{array}{l}
210 \\
211
\end{array}\right]\left[\begin{array}{ll}
100 & 000 \\
101 & 001
\end{array}\right]
$$

os arranjos $M_{0}$ e $M_{1}$ podem ser desdobrados da seguinte forma:

De $M_{0}: M_{0}:\{200\}\left\{\begin{array}{lll}110 \\ 1111\end{array}\right.$

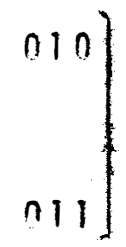

$M_{01}:[201]\left[\begin{array}{ll}110 & 010 \\ 111 & 011\end{array}\right]$

De $M_{1}: M_{10}:(210)\left[\begin{array}{ll}100 & 000 \\ 101 & 001\end{array}\right] \quad M_{11}:[211]\left[\begin{array}{ll}100 & 000 \\ 101 & 001\end{array}\right]$

Aplicando-se a sequência de diagonais DP, $D S$, DP e $D S$ em $M_{00}, M_{01}, M_{10}$ e $M_{11}$, respectivamente, onde DP e DS significam diagonal principal e diagonal secun dária, obtêm-se os blocos da primeira repetição do primeiro conjunto balanceado, que são os seguintes:

Bloco 1: 200, 110, 011

Bloco 2: $201,111,010$

Bloco 3: 210, inn, 001

B1000 4: $211,101,000$

A segunda repetição deste conjunto ë ohtida.' de maneira anälnga à primeira, com as tabelas bäsicas que formam a segunda repetição deste fatorial em hlocos de seis 
unidades. Apenas se deve tomar a sequência de diagonais $D S$, DP, DS e DP em $M_{00}, M_{01}, M_{10}$ e $M_{11}$, respectivamente.

Finalmente, a terceira repetição è obtida '

com as tabelas bäsicas que formam a terceira repetição em blocos de seis unidades, tomando-se novamente a sequência' de diagonais DP, DS, DP e DS em Moos $M_{01}, M_{10}$ e $M_{11}$.

Cada uma das três repetições do segundo conjunto balanceado são obtidas pelas sequências de diagonais: $D S, D P, D S$ e $D P ; D P, D S$, DP e $D S ; D S$, DP, DS e DP, dos ar ranjos matriciais Moo, Mol, $M_{10}$ e $M_{11}$ oriundos dos três pares de tabelas básicas que formam os blocos de seis unida des deste fatorial.

Apenas a título de ilustração mostra-se, a se guir, a primeira repetição do segundo conjunto balanceado, ou seja:

$$
\begin{aligned}
& \text { Bloco 1: 200, 111, } 010 \\
& \text { Bloco 2: } 201,110,, 11 \\
& \text { Bloco 3: 210, 101, } 000 \\
& \text { Bloco 4: } 211,100,001
\end{aligned}
$$

Cada conjunto balanceado fornece $8 / 9$ da in formação relativa em $R$, C e $C C$ e $5 / 9$ em $A R$, AC e $A B C$.

$$
\text { 4.3.3. Fatorial } 3 \times 2^{3}
$$

Neste caso, apresenta-se a construção dos fatoriais halanceados em blocos de doze e de seis unidades. 
a) Blocos de doze unidades

A partir da primeira repetição do fatorial' $3 \times 2^{2}$, em blocos de seis unidades, constrói-se o seguinte' par de tabelas:

\begin{tabular}{|c||c|c|c|c|c|c|}
\hline ABs: & 2000 & 1100 & 0100 & 2110 & 1010 & 0010 \\
\hline \hline$n n n 0$ & $200 n$ & 1100 & 0100 & 2110 & 1010 & 0010 \\
\hline $0 n n 1$ & 2001 & 1101 & 0101 & 2111 & 1011 & 0011 \\
\hline
\end{tabular}

\begin{tabular}{|c|c|c|c|c|c|c|}
\hline$A \cap C^{1}$ & 2010 & 1110 & nוח & $21 \mathrm{n}$ & $10 \cap 0$ & חמחח \\
\hline nnnn & 2010 & 1110 & 0110 & 2100 & $100 n$ & ח \\
\hline 0001 & 2011 & 1111 & $n 111$ & 2101 & 1001 & וחח \\
\hline
\end{tabular}

Forma-se um arranjo matricial com as verti cais bäsicas dessas tahelas, ou seja:

$$
\left[\begin{array}{ll}
(0000)^{0} & (000 n)^{1} \\
(0001)^{0} & (00 n 1)^{1}
\end{array}\right]
$$

As diagonais fornecem os elementos que compõem os dois blocos da primeira renotição, a seguir ilustrados:

31000 1: (nnmn) 0: 2nnn, linn, Blnn, 2110, lo1n, nnin, $(0001)^{1}: 2011,111,0111,2101,1001,0001$. 


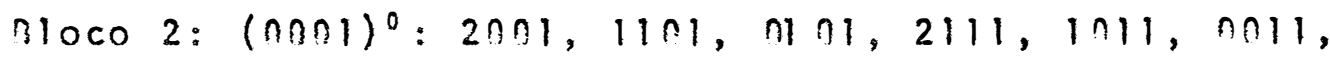
$(n n n n)^{1}: 201 n, 1110,0110,210 n, 100 n, 000 n$.

rom as tahelas häsicas oriundas da segunda e terceira repetições do fatorial $3 \times 2^{2}$, em blocos de seis $\underline{u}$ nidades, obtêm-se as duas repetições restantes do conjunto' balanceado. Este conjunto, fornece $8 / 9$ da informação relat va em $B C D$ e $5 / 9$ em ARCn.

\section{b) Blocos de seis unidades}

Podem-se obter dois conjuntos balanceados, ' sendo que cada um fornece $8 / 9$ da informação relativa nas interações $B C, B D$ e $C \cap$ e $5 / 9$ em $A \cap C, A \cap n$ e $A C D$.

Para a ohtenção dos mesmos, toma-se inicialmente os tratamentos da primeira repetição, do primeiro conJunto balanceado, do fatorial $3 \times 2^{2}$, com blocos de três uni dades, que servirão de hase para a construção das seguintes tabelas:

\begin{tabular}{|l|l|l|l|}
\hline ARC1 & $20 n n$ & $110 n$ & $n 110$ \\
\hline nnnn & $20 n n$ & $110 n$ & 0110 \\
\hline$n n n 1$ & $20 n 1$ & $11 n 1$ & $n 111$ \\
\hline
\end{tabular}

\begin{tabular}{|c||c|c|c|}
\hline$A n C^{2}$ & 2010 & 1110 & $010 n$ \\
\hline$n n n n$ & 2010 & 1110 & $n 10 n$ \\
\hline$n 001$ & 2011 & 1111 & 0101 \\
\hline
\end{tabular}

\begin{tabular}{|c||c|c|c|}
\hline$A B C^{3}$ & 2100 & $100 n$ & nnin \\
\hline$n n 0 n$ & 2100 & $100 n$ & $n 010$ \\
\hline$n n n 1$ & 2101 & 1001 & $n 011$ \\
\hline
\end{tabular}

\begin{tabular}{|c||c|c|c|}
\hline Anc & 2110 & 1010 & $n n n n$ \\
\hline$n n n n$ & $211 n$ & $\ln 10$ & $n n n n$ \\
\hline$n n n 1$ & 2111 & 1011 & $n n n 1$ \\
\hline
\end{tabular}


Para que as interações $A B$, $A C$ e $A D$, onde cada uma possui seis combinações de tratamentos, não sejam ' confundidas, isto é, para que todas as combinações dessas ' interações estejam contidas em cada bloco, deve-se formar o seguinte par de arranjos matriciais:
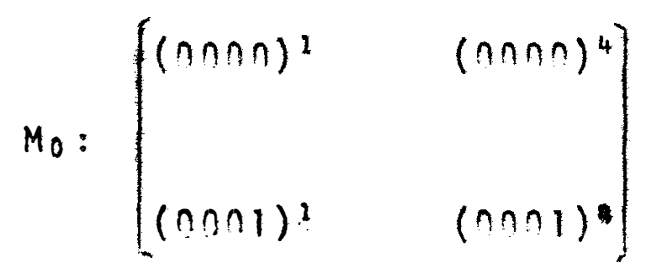

$\left.\begin{array}{l}(0000)^{3} \\ (0001)^{3}\end{array}\right]$

$(n \cap n 1)^{2}$

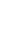

(1)

As diagonais desses arranjos fornecerão os tratamentos que constituirão os quatro blocos da primeira ' repetição do primeiro conjunto balanceado, ou seja:

Bloco 1: (nnnn) $)^{3}$ (nnnl) ${ }^{4}: 2 n n n, 11 n n, 011 n, 2111,1011,0031$ Bloco 2: (nnn1): $(n n n n)^{4}: 2 n n 1,11 n 1,0111,2110,1017,00 n 0$ Bloco $3:(n n n n)^{2} ;(n n n 1)^{3}: 2 n 1 n, 111 n, n 1 n n, 21 n 1,10 n 1,0 n 11$ Bloco 4: (nnn1) ${ }^{2} ;(n 0 n n)^{3}: 2 n 11,1111,0101,2100,10 n n, 0 n 10$ Procedendo-se de maneira anäloga com as outras duas repetições do fatorial $3 \times 2^{2}$ obtêm-se mais duas repetições, as quais, juntamente com a primeira, formam o primeiro conjunto balanceado.

n segundo conjunto balanceado é obtido repetindo-se o processo com as repetiçóes do segundo conjunto' do fatorial $3 \times 2^{2}$, com blocos de três unidades.

Em 5.3.3, apresenta-se o fatorial balanceado em blocos de doze unidades e o primeiro conjunto' balanceado em blocos de seis. 
4.4. Confundimento da série $3^{2} \times 2^{n}$

Serà apresentado o processo de confundimento dos fatoriais $3^{2} \times 2$ e $3^{2} \times 2^{2}$. No fatorial $3^{2} \times 2$, apenas' blocos de seis unidades oăo confundem efeitos principais. Contudo, blocos de nove e de três unidades serão tambèm determinados, com a finalidade de ohter os blocos de dezoito' e de seis unidades no fatorial $3^{2} \times 2^{2}$.

4.4.1. Fatorial $3^{2} \times 2$

A obtenção de blocos de nove, seis e três unidades, deste fatorial, pode ser efetuada através do fatorial $3^{2}$. Entretanto, os blocos de nove unidades, bem como os de três, que confundem o efeito principal c, em dois niveis, săo mais facilmente obtidos, com economia de repetições, atravès do fatorial $3 \times 2$.

a) Blocos de nove unidates

Através das tabelas bäsicas que podem ser' formadas com os tratamentos do fatorial $3^{2}$, em blocos de três unidades, Dode-se obter um conjunto balanceado com seis repetições, o qual confunde o efeito principal c e a interação ARC. Entretanto, ap̧enas três repetiçōes serão neces 
särias ao se formar blocos de nove unidades com os tratamen tos do fatorial $3 \times 2$.

Da primeira repetição do fatorial $3 \times 2$ forma-se o seguinte par de tabelas, acrescentando-se nas verti cais bäsicas, os niveis do fator $A$.

\begin{tabular}{|c||c|c|c|}
\hline$B C^{\circ}$ & 020 & 011 & 001 \\
\hline 000 & 020 & 011 & 001 \\
\hline 100 & 120 & 111 & 101 \\
\hline 200 & 220 & 211 & 201 \\
\hline
\end{tabular}

\begin{tabular}{|c||c|c|c|}
\hline$B C^{1}$ & 021 & 010 & 000 \\
\hline 000 & 021 & 010 & 000 \\
\hline 100 & 121 & 110 & 100 \\
\hline 200 & 221 & 210 & 200 \\
\hline
\end{tabular}

is tratamentos de cada tabela formam um bloco do fatorial $3^{2} \times 2$, ou seja:

Bloco 1: 020, 011, 001, 120, 111, 101, 220, 211, 201 Bloco 2: $021,010,0 n n, 121,110,10 n, 221,210,200$

Com os tratamentos das outras duas repetiçōes do fatorial $3 \times 2$, obtêm-se mais dois pares de tabelas, dos quais resultam as duas repetições restantes do conjunto ba lanceado.

Como não hä operações entre tahelas para a ob tenção dos blocos, a informação relativa e os efeitos fator ais confundidos são os mesmos do fatorial $3 \times 2$, ou seja:8/9 para o fator c e $5 / 9$ para a interação $7 C$.

romo se pode verificar, a única desvantagem' desse conjunto balanceado, em relação àquele que pode ser 
formado com o fatorial $3^{2}$, è que este confunde uma intera ção de dols fatores, além do efeito principal $c$, e aquele, a interação tripla.

b) Blocos de seis unitares

Com hase na primeira repetição do fatorial' $3^{2}$ formam-se as seguintes tabelas básicas:

\begin{tabular}{|c||c|c|c|}
\hline$A 0^{\circ}$ & 200 & 110 & 020 \\
\hline 000 & 200 & 110 & 020 \\
\hline 001 & 201 & 111 & 021 \\
\hline
\end{tabular}

\begin{tabular}{|c||c|c|c|}
\hline$A B^{1}$ & 210 & 120 & $00 n$ \\
\hline$n n n$ & 210 & 120 & 000 \\
\hline$n n 1$ & 211 & 121 & 001 \\
\hline
\end{tabular}

\begin{tabular}{|c|c|c|c|}
\hline$A B^{2}$ & 220 & 100 & 010 \\
\hline $00 n$ & 220 & 100 & $n 10$ \\
\hline $0 n 1$ & 221 & 101 & 011 \\
\hline
\end{tabular}

Combinando-se as verticais häsicas de cada tä bela, duas a duas, ohtêm-se três arranjos matriciais, tais co mo: 
67.

$$
\begin{aligned}
& M_{0}:\left[\begin{array}{ll}
(n n n)^{0} & (0 n n)^{2} \\
(n n 1)^{0} & (n n 1)^{1}
\end{array}\right] \\
& M_{1}:\left[\begin{array}{ll}
(n n n)^{0} & (n n n)^{2} \\
(n n 1)^{0} & (n n 1)^{2}
\end{array}\right] \\
& M_{2}:\left[\begin{array}{ll}
(n n n)^{1} & (n n n)^{2} \\
(n n 1)^{1} & (0 n 1)^{2}
\end{array}\right]
\end{aligned}
$$

Os três bincos da primeira repetição são obtidos pela sequêencia de diagonais DP, DS e DP em $M_{0}, M_{1}$ e $M_{2}$, respectivamente, que são:

Bloco 1: $(n n n)^{0} ;(n n 1)^{1}: 2 n n, 11 n, n 2 n, 211,121, n n$

Bloco 2: $(n n 1)^{0} ;(n n n)^{2}: 2 n 1,111, n 21,22 n, 1 n n, 010$

Bloco 3: $(n n n)^{1} ;(n n 1)^{2}: 2.1 n, 12 n, 3 n n, 221,101, n 11$

A segunda repetição resulta da sequéncia de diagonais DS, DP e DS, respectivamente em $M_{0}, M_{1}$ e $M_{2}$.

Mais duas repetições são obtidas com as tabe las provenientes da segunda repetição do fatorial $3^{2}$.

A informação relativa ohtida nas interações' confundidas desse conjunto balanceado è de $7 / 8$ em $A$ ? e $5 / 8$ em $A B C$. 


\section{c) Blocos de três unidades}

Considerando cada tabela bäsica que deu origem aos hlocos de nove unidades deste fatorial, como a de um efeito principal do fatorial $3^{2}$, isto é, desdobrando-a ' em três tabelas, pode-se aplicar o processo de confundimento daquele fatorial, donde se obtém.rs blocos de três unida des.

Efetivamente, através do par de tabelas dadas no item a) podem-se formar os seguintes arranjos matriciais:

De $B C^{0}$ :

$M_{00}:[n 2 n]\left[\begin{array}{cc}111 & 101 \\ 211 & 201\end{array}\right) \quad M_{02}:\left\{\begin{array}{ll}12 & n\end{array}\right]\left(\begin{array}{ll}211 & 201 \\ n 11 & n n 1\end{array}\right) \quad M_{02}:[22 n]\left(\begin{array}{ll}n 11 & 0 n 1 \\ 111 & 101\end{array}\right)$ De $B C^{1}$ :

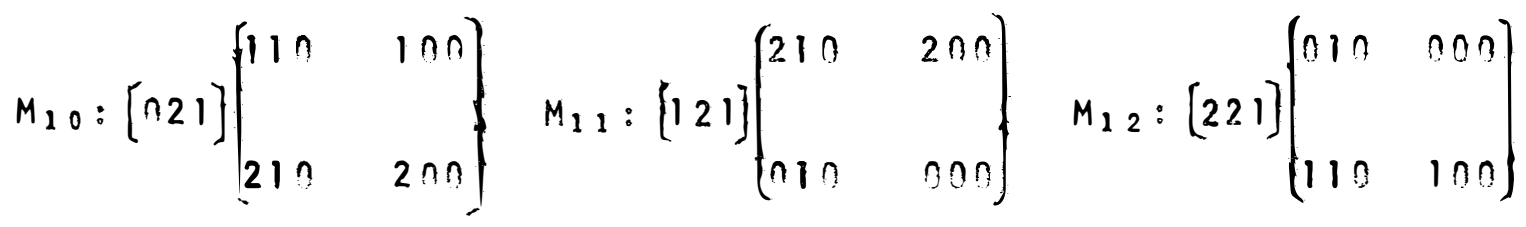

A primeira repetição deste fatorial é obtida então através das diagonais principais dos seis arranjos ma triciais, lembrando que se deve acrescentar a combinação em separado. Procedendo-se dessa maneira obtèm-se:

Bloco 1: $32 n, 111,2 n 1$

Bloco 2: $12 n, 211, n n 1$ 
Bloco $3: 22 n, 011,101$

Bloco $4: n 21,11 n, 2 n n$

Bloco $5: 121,210,0 n \pi$

Bloco 6:221, nin, inn

A segunda repetição resulta das diagonais se cundàrias.

Com os outros dois pares de tahelas que originam a segunda e terceira repetições em blocos de nove uni dades deste fatorial, ohtēm-se mais quatro repetições, por processo anälogo.

O fatorial balanceado, composto das seis repetições, fornece $8 / 9$ da informação relativa no efeito prín cipal C, 1/2 em $A B, 5 / 9$ em $A C$ e $B C$ e $13 / 18$ em $A B C$.

ns conjuntos balanceados em blocos de nove, de seis e de três unidades encontram-se em 5.4.1 de Resulta dös.

4.4.2. Fatorial $3^{2} \times 2^{2}$

Para este esquema, apresentar-se-ä a constrü ção dos conjuntos balanceados em blocos de dezoito, de nove e de seis unidades. 


\section{a) Blocos de dezoito unidades}

Baseando-se na primeira repetição do fatoria) $3^{2} \times 2$, em blocos de nove unidades, constrói-se o par de tabelas básicas seguinte:

\begin{tabular}{|c|c|c|c|c|c|c|c|c|c|}
\hline$A B C^{\circ}$ & $32 n 0$ & חו & nחוn & $12 n n$ & 1110 & 1010 & $220 n$ & 2110 & $2 n 1 n$ \\
\hline 0000 & $020 n$ & חווn & noln & 1200 & 1110 & 1010 & 2200 & 2110 & 2010 \\
\hline Inח & $n 201$ & 0111 & 'ו & 1201 & 1111 & 1011 & $22 n 1$ & 2111 & 2011 \\
\hline
\end{tabular}

\begin{tabular}{|c|c|c|c|c|c|c|c|c|c|}
\hline$A B C^{1}$ & 7217 & n & nחn & 1210 & 1100 & Inח? & 2210 & 2100 & 2000 \\
\hline $0 n n n$ & $021 ?$ & 0100 & חחחר & 1210 & 1100 & $10 n n$ & 2210 & 2100 & 2000 \\
\hline 0001 & 0211 & וחוח & Iחח & 1211 & 1101 & $\ln 1$ & 2211 & $21 \cap 1$ & 2001 \\
\hline
\end{tabular}

Com as verticais häsicas desse par de tabe las monta-se o seguinte arranjo matricial:

$$
\left[\begin{array}{ll}
(\operatorname{nnnn})^{0} & (\text { nnon })^{1} \\
(n \operatorname{non})^{0} & (0 n n 1)^{1}
\end{array}\right]
$$

que, atravēs das diagonais, fornecerá os tratamentos que' formarão os dois blocos da primeira repetição:

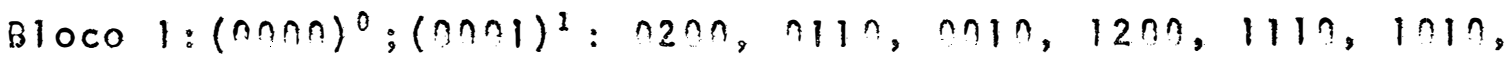
$22 n n, 211^{n}, 2 n 1 n, 0211,0101,00 n 1$, $1211,1101,1 n n 1,2211,21 n 1,2 n n 1$. 
Bloco 2: (nnn1) 0 (nnnn) $)^{1}: 02 n 1,0111,0011,1201,1111,1011$, $22 n 1,2111,2 n 11, n 21 n, n 10 n, 00 n n$, $121 n, 11 n n, 10 n n, 2210,21 n n, 2 n n \pi$.

De maneira anäloga, mais duas repetições são obtidas a partir das tabelas básicas construidas em função' da segunda e da terceira repetições do fatorial $3^{2} \times 2$ em blocos de nove unidades.

O fatorial balanceado fornece $8 / 9$ da informação relativa na interação con e $5 / 9$ em Bron.

b) Blocos de doze unidades

Dito repetições formam o fatorial balanceado fornecendo $31 / 32$ da informação relativa em $A B, 29 / 32$ em $A B C$ e $A B O$ e 23/32 em $A R C D$.

As repetições 1 e 11 são ohtidas com as três tabelas bäsicas formadas pelos tratamentos da primeira repe tição do fatorial $3^{2} \times 2 \mathrm{em}$ hlocos de seis unidades:

\begin{tabular}{|c|c|c|c|c|c|c|}
\hline$A B C^{\circ}$ & 2000 & 1100 & 0200 & 2110 & 1210 & 0010 \\
\hline 0000 & 2000 & 1100 & 0200 & 2110 & 1210 & 0010 \\
\hline 0001 & 2001 & 1101 & 0201 & 2111 & 1211 & 0011 \\
\hline
\end{tabular}


72 .

\begin{tabular}{|c|c|c|c|c|c|c|}
\hline$A B C^{1}$ & 2010 & 1110 & 0210 & 2200 & 1000 & 0100 \\
\hline 0000 & 2010 & 1110 & 0210 & 2200 & 1000 & 0100 \\
\hline 0001 & 2011 & 1111 & 0211 & 2201 & 1001 & 0101 \\
\hline
\end{tabular}

\begin{tabular}{|l|l|l|l|l|l|l|}
\hline$A B C^{2}$ & 2100 & 1200 & 0000 & 2210 & 1010 & 0110 \\
\hline 0000 & 2100 & 1200 & 0000 & 2210 & 1010 & 0110 \\
\hline 0001 & 2101 & 1201 & 0001 & 2211 & 1011 & 0111 \\
\hline
\end{tabular}

A seguir, tomam-se as verticais bäsicas de cada tabela para compor três arranjos matriciais, resultantes ' da combinação das verticais bäsicas, tomadas duas a duas. os arranjos são os seguintes:

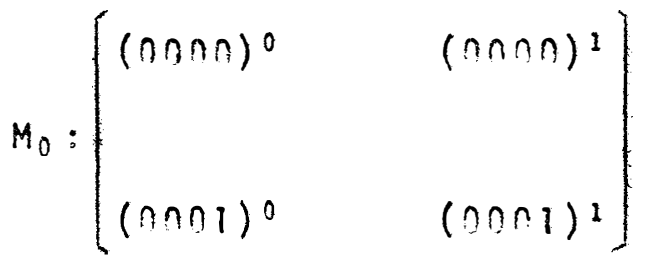

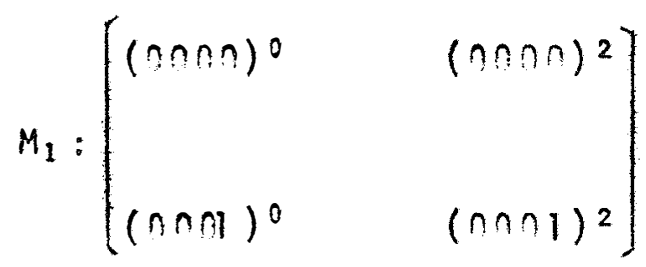

$$
\begin{aligned}
& M_{2}:\left[\begin{array}{ll}
(n n 00)^{1} & (\text { nnn })^{2} \\
(n n n 1)^{1} & (\text { nn } 1)^{2}
\end{array}\right]
\end{aligned}
$$


Pela sequência de diagonais $D P$, $D S$ e $D P$ em $M_{0}, M_{1}$ e $M_{2}$, respectivamente, obtëm-se os blocos da prime ra repetição que são:

Bloco 1: $(0000)^{0}: 2000,1100,0200,2110,1210,0010$, $(0 n n 1)^{1}: 2 n 11,1111, n 211,2201,10 n 1, n 101$.

Bloco 2: $(n 001)^{0}: 2001,11 n 1, n 2 n 1,2111,1211, n n 11$, $(000 n)^{2}: 2100,1200, n 0 n \pi, 2210,1010,0110$.

Bloco 3: $(n n n n)^{3}: 2 n 1 n, 111 n, n 21 n, 220 n, 10 n n, 010 n$, $(n n n 1)^{2}: 21 n 1,12 n 1,0 n n 1,2211,1011,0111$.

A segunda repetição resulta das diagonais rs, DP e DS em $M_{0}, M_{1}$ e $M_{2}$, respectivamente.

Formando-se as tabelas bäsicas com as repetições 11,111 e IV do fatorial $3^{2} \times 2$ em blocos de seis unida des e aplicando-se o mesmo processo, obtém-se as seis repeti ções restantes.

;) Blocos de seis unidades

O fatorial balanceado, neste caso, consta de seis repetições. Cada repetição ë proveniente das tabelas bä sicas formadas com os tratamentos do fatorial $3^{2} \times 2$ em blocos de três unidades.

Tomando-se a primeira repetição daquele fatorial formam-se as seis tabelas bäsicas: 


\begin{tabular}{|c|c|c|c|}
\hline$A B C^{1}$ & $n 2 n n$ & $111 n$ & $2 n 1 n$ \\
\hline$n n n n$ & $n 2 n n$ & 1110 & 2010 \\
\hline$n n n 1$ & 0201 & 1111 & $2 n 11$ \\
\hline
\end{tabular}

\begin{tabular}{|c|c|c|c|}
\hline$A B C^{2}$ & $12 n n$ & 2110 & $0 n 10$ \\
\hline$n n n n$ & $12 n n$ & $211 n$ & $0 n 10$ \\
\hline$n n n 1$ & 1201 & 2111 & $0 n 11$ \\
\hline
\end{tabular}

\begin{tabular}{|l|l|l|l|}
\hline$A B C^{3}$ & $220 n$ & 0110 & 1010 \\
\hline $0 n 0 n$ & $22 n n$ & 0110 & 1010 \\
\hline$n n n 1$ & $22 n 1$ & 0111 & 1011 \\
\hline
\end{tabular}

\begin{tabular}{|c|c|c|c|}
\hline$A B C^{4}$ & $n 210$ & 1100 & 2000 \\
\hline$n n n n$ & 0210 & 1100 & $200 n$ \\
\hline$n n n 1$ & $n 211$ & 1101 & $20 n 1$ \\
\hline
\end{tabular}

\begin{tabular}{|c|c|c|c|}
\hline$A B C^{5}$ & 1210 & 2100 & $n 0 n n$ \\
\hline 0000 & 1210 & 2100 & 0000 \\
\hline 0001 & 1211 & 2101 & $n 001$ \\
\hline
\end{tabular}

\begin{tabular}{|c||c|c|c|}
\hline$A B C^{6}$ & 2210 & 0100 & 1000 \\
\hline 0000 & 2210 & 0100 & 1000 \\
\hline 0001 & 2211 & 0101 & 1000 \\
\hline
\end{tabular}

A fim de evitar o confundimento das interações $A C, A D, B C$ e $B D$, as quais possuem seis niveis, deve-se formar os arranjos matriciais como a seguir:

$$
\begin{aligned}
& M_{0}:\left[\begin{array}{ll}
(n n 00)^{1} & (n n n n)^{4} \\
(n n n 1)^{1} & (n n 01)^{4}
\end{array}\right] \\
& M_{1}:\left[\begin{array}{ll}
(n n 00)^{2} & (n n n n)^{5} \\
(n n n 1)^{2} & (n n n 1)^{5}
\end{array}\right]
\end{aligned}
$$




$$
M_{2}:\left[\begin{array}{ll}
(\operatorname{nnnn})^{3} & (\text { nnno })^{6} \\
(\operatorname{nnn} 1)^{3} & (\text { nnn } 1)^{6}
\end{array}\right]
$$

As diagonais de cada arranjo formarão os blocos da primeira repetição que são assim constituidos: Bloco 1: $(0000)^{1} ;(0001)^{4}: 0200,1110,2010,0211,1101,2001$ Bloco 2: $(n n n 1)^{1} ;(00 n n)^{4}: 02 n 1,1111,2011,0210,110 n, 2 n n n$ Bloco 3: $(n n n n)^{2} ;(n n n 1)^{5}: 12 n n, 211 n, 0 n 10,1211,2101,0001$ Bloco 4: $(0 n n 1)^{2} ;(n n n n)^{5}: 12 n 1,2111, n n 11,121 n, 210 n, 0 n 0 n$ Bloco 5: $(n n n n)^{3} ;(n n n l)^{6}: 22 n n$, niln, inin, 2211, ninl, inn Bloco 6: $(n n n 1)^{3} ;(n n n n)^{6}: 22 n 1, n 111,1 n 11,221 n$, ninn, $130 n$ procedendo-se de maneira anäloga com as demais repetições do fatorial $3^{2} \times 2$, em blocos de três unidades, ob tëm-se o conjunto balanceado.

0 conjunto balanceado fornece $1 / 2$ da informa ção relativa na interação $A B, 8 / 9$ em C.D, 5/9 em $A C$ e $13 / 18$ na interação $A B C, D$.

Em 5.4.2. de Resultados, encontram-se os con juntos balanceados em blocos de dezoito, de nove e de seis unidades. 
4.5. Confundimento da série $3^{3} \times 2^{n}$

Apresentar-se-ä apenas o processo de confund $\underline{\mathbf{i}}$ mento do fatorial $3^{3} \times 2$, em razão do nümero excessivamente' grande de tratamentos que compõem os outros fatoriais desta sèrie.

\subsubsection{Fatorial $3^{3} \times 2$}

0 confundimento deste fatorial ë feito a partir do fatorial $3^{3}$. Pode-se obter blocos de dezoito unidades construindo-se tabelas bäsicas a partir dos blocos de nove tratamentos do fatorial $3^{3}$ e blocos de seis unidades com as tabelas formadas atravēs dos blocos de três parcelas daquele fatorial.

a) Blocos de dezolto unidades

Para este caso, oito repetições são necessä. rias para a obtenção do conjunto balanceado. As tabelas bás cas formadas com uma repetição do fatorial $3^{3}$, em blocos de nove unidades, fornecem duas repetiçöes para este fatorial. A tỉtulo de ilustração,apresenta-se as tabelas bäsicas forma das com a primeira repetiçãn do fatorial $3^{3}$, ou sejam: 


\begin{tabular}{|c||c|c|c|c|c|c|c|c|c|}
\hline$A B C^{\circ}$ & 2000 & 1100 & $020 n$ & 2110 & 1210 & 0010 & 2220 & 1020 & 0120 \\
\hline $0 n n n$ & $20 n 0$ & $110 n$ & $020 n$ & 2110 & 1210 & $0 n 10$ & 2220 & 1020 & 0120 \\
\hline$n 0 n 1$ & $20 n 1$ & 1101 & 0201 & 2111 & 1211 & 0011 & 2221 & 1021 & 0121 \\
\hline
\end{tabular}

\begin{tabular}{|c|c|c|c|c|c|c|c|c|c|}
\hline$A B C^{1}$ & $2 n 10$ & 1110 & 0210 & 2121 & $122 n$ & $n n 2 n$ & $22 n n$ & $10 n 0$ & 0100 \\
\hline 0000 & $2 n 10$ & 1111 & 0210 & 2120 & $122 n$ & $n n 2 n$ & $22 n n$ & $10 n n$ & $n 10 n$ \\
\hline 0001 & $2 n 11$ & 1111 & $n 211$ & 2121 & 1221 & $n n 21$ & 2201 & $10 n 1$ & $n 101$ \\
\hline
\end{tabular}

\begin{tabular}{|c|c|c|c|c|c|c|c|c|c|}
\hline$A B C^{2}$ & $2 \cap 2 n$ & 1120 & 0220 & $210 n$ & $120 n$ & กกก & 2210 & $\ln 10$ & 0110 \\
\hline$n \cap 00$ & $2 \cap 2 \cap$ & 1120 & $022 \cap$ & $21 \cap 0$ & 1200 & nח00 & 2210 & 1010 & 0110 \\
\hline ו & $2 \cap 21$ & 1121 & $n 221$ & 2101 & 1201 & ו & 2211 & 1011 & 0111 \\
\hline
\end{tabular}

Combinando-se as verticais bäsicas de cada tä bela, duas a duas, formamose os seguintes arranjos matrici ais:

$$
\begin{aligned}
& M_{0}:\left[\begin{array}{ll}
(\text { onnn })^{0} & (\text { nono })^{2} \\
(\text { nnnl })^{0} & (\text { nnn })^{2}
\end{array}\right]
\end{aligned}
$$

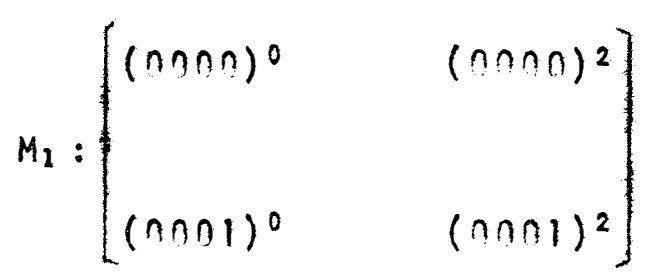




$$
M_{2}:\left[\begin{array}{ll}
(0 n n 0)^{1} & (00 n n)^{2} \\
(0001)^{1} & (0 n n 1)^{2}
\end{array}\right]
$$

A primeira repetição deste fatorial é obtida aplicando-se a seqüencia de diagonais DP, DS e DP nos arran jos $M_{0}, M_{1}$ e $M_{2}$, cujos blocos são assim constituidos:

Bloco 1: (0nno) $0: 2 n n n, 11 n n, 02 n n, 2110,1210,0 n 1 n, 222 n, 102 n, 012 n$, $(n 0 n 1)^{1}: 2 n 11,1111, n 211,2121,1221, n n 21,22 n 1,1 n n 1, n 1 n 1$.

Bloco 2: (nnח1) $0: 2001,11 n 1, n 2 n 1,2111,1211,0011,2221,1021,0121$, $(n n n 0)^{2}: 2 n 2 n, 1120, n 22 n, 21 n n, 12 n n, n 0 n n, 2210,1010,011 n$. Bloco $3:(00 n n)^{2}: 2 n 1 n, 111 n, n 21 n, 212 n, 122 n, n n 2 n, 22 n n, 100 n, n 1 n n$, $(n n n 1)^{2}: 2 n 21,1121,0221,21 \cap 1,12 n 1,0 n n 1,2211,1 n 11,0111$.

A segunda repetição resulta da sequêneia de diagonais DS, DP e DS em $M_{0}, M_{1}$ e $M_{2}$, respectivamente.

Através das tabelas bäsicas formadas com as outras três repetições do fatorial $3^{3}$, em blocos de nove un dades, obtém-se mais seis repetições.

0 conjunto halanceado fornece $15 / 16$ da informaçäo relativa em $A \cap r$ e $13 / 16$ na interação $A B C D$.

b) Blocos de seis unidades

Um conjunto balanceado, o qual é composto' de oito repetições e fornecendo $7 / 8$ da informação relativa' 
nas interações $A B, A C$ e $B C, 3 / 4$ em $A R C$ e $5 / 8$ em $A B D, A C D$ e BCD, è possivel de ser formado seguindo-se o processo que será descrito a seguir:

Conströi-se inicialmente as nove tabelas bäsicas seguintes, cujas horizontais são provenlentes da primeira repetição do fatorial $3^{3}$ em blocos de três unidades. Nota-se que, essas nove tahelas, estão ordenadas, em ordem crescente, de acordo com a nrimelra combinação de cada hor zontal bäsica.

\begin{tabular}{|c|c|c|c|}
\hline$A B C^{00}$ & 2000 & 1110 & 0220 \\
\hline 0000 & 2000 & 1110 & 0220 \\
\hline 0001 & 2001 & 1111 & 0221 \\
\hline
\end{tabular}

\begin{tabular}{|c||c|c|c|}
\hline$A B 0^{01}$ & 2010 & 1120 & 0200 \\
\hline 0000 & 2010 & 1120 & 0200 \\
\hline 0001 & 2011 & 1121 & 0201 \\
\hline
\end{tabular}

\begin{tabular}{|c||c|c|c|}
\hline$A B C^{02}$ & 2020 & 1100 & 0210 \\
\hline 0000 & 2020 & 1100 & 0210 \\
\hline $0 n 01$ & 2021 & 1101 & 0211 \\
\hline
\end{tabular}

\begin{tabular}{|c|c|c|c|}
\hline$A B C^{2}$ & $210 n$ & 1210 & 0020 \\
\hline 0000 & 2100 & 1210 & $0 n 20$ \\
\hline 0001 & 2101 & 1211 & 0021 \\
\hline
\end{tabular}

\begin{tabular}{|c||c|c|c|}
\hline$A B C^{11}$ & 2110 & 1220 & 0000 \\
\hline 0000 & 2110 & 1220 & $n 030$ \\
\hline 0001 & 2111 & 1221 & 0001 \\
\hline
\end{tabular}

\begin{tabular}{|c||c|c|c|}
\hline$A B C^{12}$ & 2120 & 1200 & 0010 \\
\hline$n n n n$ & 2120 & 1200 & $0 n 10$ \\
\hline$n n n 1$ & 2121 & $12 n 1$ & $n n 11$ \\
\hline
\end{tabular}

\begin{tabular}{|c|c|c|c|}
\hline$A B C^{20}$ & 2200 & 1010 & 0120 \\
\hline $0 n 00$ & 2200 & 1010 & 0120 \\
\hline 0001 & 2201 & 1011 & 0121 \\
\hline
\end{tabular}

\begin{tabular}{|c||c|c|c|}
\hline$A n C^{21}$ & 2210 & 1020 & 0100 \\
\hline 0000 & 2210 & 1020 & 0100 \\
\hline 0001 & 2211 & 1021 & 0101 \\
\hline
\end{tabular}




\begin{tabular}{|l||c|c|c|}
\hline$A B C^{22}$ & 2220 & 1000 & 0110 \\
\hline $0 n 00$ & 2220 & 1000 & $n 110$ \\
\hline$n n n 1$ & 2221 & 1001 & 0111 \\
\hline
\end{tabular}

os indices de $A B C$, das tabelas bäsicas,correspondem aos niveis de $B$ e C da primeira combinação de cada ho rizontal bäsica e formam os tratamentos do fatorial $3^{2}$, de mo do que se pode combinar estas tabelas, três a três, de acordo com as repetições daquele fatorial, ou sejam:

"REPETICAO I"
a) $A B C^{00} ; A B C^{11} ; A \cap C^{22}$
d) $A B C^{0} ; A B C^{21} ; A B C^{12}$
b) $A B C^{10} ; A B C^{21} ; A B C^{02}$
e) $A B C^{10} ; A B C^{01} ; A B C^{22}$
c) $A B C^{20} ; A B C^{01} ; A B C^{12}$
f) $A B C^{20} ; A B C^{11} ; A B C^{02}$ as a duas, das tabelas correspondentes ao item d) da "Repeti ção 11 ", formam-se os três arranjos matriciais seguintes:

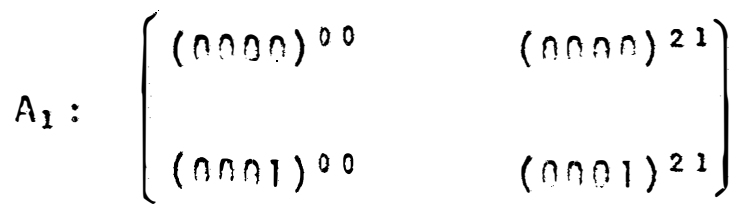

$$
\begin{aligned}
& A_{2}:\left(\begin{array}{ll}
(000 n)^{00} & (0 n n \cap)^{12} \\
(0 n n 1) 00 & (n n \cap 1)^{12}
\end{array}\right) \\
& A_{3}=\left[\begin{array}{ll}
(0000)^{21} & (0000)^{12} \\
(0001)^{21} & (0001)^{12}
\end{array}\right]
\end{aligned}
$$


A sequência de diagonais or, os e oP de $A_{1}$, $A_{2}$ e $A_{3}$, respectivamente, determinam os três primeiros blocos da primeira repetição, do primeiro conjunto balanceado, ou sejam:

Bloco 1: $(n n 00) 00 ;(n n n 1)^{21}: 20 n 0,1110,0220,2211,1021,0101$

Bloco 2: $(n n n 1)^{08} \quad(n n 00)^{12}: 2 n n 1,1111,0221,2120,1200,0010$

B1000 3: $(0000)^{21 ;} ;(0001)^{12}: 2210,1020,0100,2121,1201,0011$

Formando-se os arranjos matriciais com as '

verticais bäsicas das tabelas de e) e f) obtēm-se os seis blocos restantes.

A segunda repetição resulta da sequência de diagonais DS, Of e OS nos arranjos matriciais construidos' para a obtenção da nrimeira.

Construindo-se as nove tabelas häsicas com a segunda repetição do fatorial $3^{3}$, em blocos de nove unidades e repetindo-se o processo com as tabelas ordenadas três a três,de acordo com a "renetição l", obtēm-se as repetiçöes III e IV.

As repetições $V$ e VI são obtidas com as nove tabelas básicas provenientes da terceira repetição do fatorial $3^{3}$, tambēm ordenadas de acordo com a "renetição l".

Finalmente, as repetições VII e VIII resul tam das nove tabelas bảsicas formadas com os tratamentos da quarta repetição do fatorial $3^{3}$, ordenadas atravàs da "rene tição $11 "$. 
o conjunto halanceado em blocos de dezoito $\underline{u}$ nidades e o primeiro conjunto halanceado em blocos de seis, são dados em 5.5 .1 de Resultados.

4.6. Processo de obtenção da informação relativa

Apresentar-se-á o processo de determinação' da variancia dos efeitos fatoriais e o de obtenção da infor mação relativa através da teoria de blocos incompletos.

No Apêndice deste trabalho é dado o programa em linguagem FORTRAN para o computador $18 M-1130$, com suas limitações, bem como as instruções para seu uso, acompanhadas de um exemplo.

4.6.1. Sistema de equações normais para efeitos de tratamentos estimados

Considere-se como exemplo as três repetições do conjunto balanceado do fatorial $3 \times 2$ cujo plano "e dado em 5.3.1 de Resultados, onde os blocos são assim constituidos:

\begin{tabular}{|c|c|c|c|}
\hline Bloco 1 & 20 & 11 & 01 \\
\hline 810002 & 21 & 10 & 30 \\
\hline 810003 & 10 & 21 & 01 \\
\hline Bloco 4 & 11 & 20 & 00 \\
\hline
\end{tabular}




\begin{tabular}{|c|c|c|c|}
\hline Bloco 5 & $n n$ & 21 & 11 \\
\hline 310006 & 01 & $2 n$ & 10 \\
\hline
\end{tabular}

Da teoria de blocos incompletos sabe-se que - sistema de equaçōes normais para efoltos de tratamentos' estimados è dado por:

$$
\hat{C T}=0
$$

onde $C$ é uma matriz quadrada de ordem $v(v=$ nümero de tratamentos), cujos elementos $c_{i j}$ são:

$$
\begin{aligned}
& c_{i j}=(1-1 / k) r \\
& c_{i j}=-\lambda_{i j} / k
\end{aligned}
$$

sendo $\underline{k}$ o tamanho dos blocos; $\underline{r}$, o nümero de repetições e $\underline{\lambda}_{i j}$ o nümero de vezes que os tratamentos $\underline{i}$ e $\mathfrak{I}$ aparecem ' juntos nos blocos.

A matriz $\hat{i}$ è a matriz dos efeitos de trata mentos estimados, que neste exemplo são formados pelos niveis dos fatores e são dispostos, na matriz, em ordem crescente. A ordem dessa matriz è $v \times 1$.

Os elementos $C_{i}$ da matriz 0 , de ordem $v \times 1$, são:

$$
n_{i}=T_{i}-\frac{1}{k} A_{i}
$$

onde $T_{i}$ é o total do i-ésimo tratamento e $A_{i} \dot{e}$ o total dos blocos onde aparece o tratamento $i$. 
o sistema de equações normais, para o exem plo em pauta é. o seguinte:

$$
\left[\begin{array}{cccccc}
2 & 0 & -1 / 3 & -2 / 3 & -1 / 3 & -2 / 3 \\
0 & 2 & -2 / 3 & -1 / 2 & -2 / 3 & -1 / 3 \\
-1 / 3 & -2 / 3 & 2 & 0 & -1 / 3 & -2 / 3 \\
-2 / 3 & -1 / 3 & 0 & 2 & -2 / 3 & -1 / 3 \\
-1 / 3 & -2 / 3 & -1 / 3 & -2 / 3 & 2 & 0 \\
-2 / 3 & -1 / 3 & -2 / 3 & -1 / 3 & 0 & 2
\end{array}\right]\left[\begin{array}{c}
(\hat{0} \hat{)} \\
(\hat{0 i}) \\
(\hat{i}) \\
(\hat{i}) \\
(2 \hat{0}) \\
(2 \hat{i})
\end{array}\right]=\left[\begin{array}{l}
0_{0} \\
0_{01} \\
0_{10} \\
0_{11} \\
0_{20} \\
0_{21}
\end{array}\right]
$$

4.6.2. Obtenção da matriz de dispersão

Por ser a matriz C singular, deve-se tomar ' uma matriz $A$, tal que $\hat{A T}=\phi$, sendo $A$ de tal forma que, fazendo $M=C-A$, esta seja inversível.

Infelizmente, os conjuntos balanceados nos' esquemas fatoriais não correspondem aos blocos incompletos' balanceados, nos quals, atravës de uma matriz $A$, de restrição, conveniente, obtēm-se a matriz M diagonal, cuja inversão è elementar.

Nos fatoriais balanceados não è possivel obter a matriz M diagonal. Como estes geralmente envolvem um grande nümero de tratamentos, o processo só è viāvel atravēs de computador.

Para o exemplo, pode-se tomar como restrição: 
$\frac{1}{3}[(\hat{0} 0)+(\hat{0})+\ldots+(2 i)]=0$. Dessa forma, a matriz A serä:

$$
A=-\frac{1}{3}\left[\begin{array}{llllll}
1 & 1 & 1 & 1 & 1 & 1 \\
1 & 1 & 1 & 1 & 1 & 1 \\
1 & 1 & 1 & 1 & 1 & 1 \\
1 & 1 & 1 & 1 & 1 & 1 \\
1 & 1 & 1 & 1 & 1 & 1 \\
1 & 1 & 1 & 1 & 1 & 1
\end{array}\right]
$$

e a matriz M:

$$
M=\frac{1}{3}\left[\begin{array}{rrrrrr}
7 & 1 & 0 & -1 & 0 & -1 \\
1 & 7 & -1 & 0 & -1 & 0 \\
0 & -1 & 7 & 1 & 0 & -1 \\
-1 & 0 & 1 & 7 & -1 & 0 \\
0 & -1 & 0 & -1 & 7 & 1 \\
-1 & 0 & -1 & 0 & 1 & 7
\end{array}\right]
$$

A matriz de dispersão, como se sabe, è dada'

por:

$$
0=M^{-1} C M^{-1} \delta^{2}
$$

Logo, precisa-se conhecer $M^{-1}$, ou seja: 


$$
M^{-1}=\frac{1}{720}\left[\begin{array}{rrrrrr}
329 & -49 & -7 & 47 & -7 & 47 \\
-49 & 329 & 47 & -7 & 47 & -7 \\
-7 & 47 & 329 & -49 & -7 & 47 \\
47 & -7 & -49 & 329 & 47 & -7 \\
-7 & 47 & -7 & 47 & 329 & -49 \\
47 & -7 & 47 & -7 & -49 & 329
\end{array}\right]
$$

Efetuando-se o produto $M^{-1} C M^{1^{-1}}$, obtèm-se:

$$
D=\frac{1}{720}\left(\begin{array}{rrrrrr}
269 & -109 & -67 & -13 & -67 & -13 \\
-109 & 269 & -13 & -67 & -13 & -67 \\
-67 & -13 & 269 & -109 & -67 & -13 \\
-13 & -67 & -109 & 269 & -13 & -67 \\
-67 & -13 & -67 & -13 & 269 & -109 \\
-13 & -67 & -13 & -67 & -109 & 269
\end{array}\right) \delta^{2}
$$

Os elementos da diagonal desta matriz forne cem as variâncias dos tratamentos e os elementos fora da dia gonal, as covariâncias. Assim, para o exemplo, tem-se:

$$
v(\hat{00})=v(\hat{0 i})=\ldots=v(2 \hat{i})=\frac{269}{720} \delta^{2}=0,3736111 \delta^{2}
$$

$\operatorname{cov}(\hat{O O O}, \hat{1})=\operatorname{cov}(\hat{0}, \hat{1})=\operatorname{cov}(2 \hat{\pi}, 2 \hat{i})=-\frac{109}{72} \delta^{2}=-0,1513888 \delta^{2}$

$$
\operatorname{cov}(\hat{0}, \hat{0})=\operatorname{cov}(\hat{00}, \hat{n})=\operatorname{cov}(\hat{0}, \hat{i})=\operatorname{cov}(\hat{0}, \hat{i})=
$$

$=\operatorname{Cov}(\hat{i n}, \hat{0})=\operatorname{Cov}(\hat{i}, \hat{2 i})=-\frac{67}{720} \sigma^{2}=-0,0930555 \delta^{2}$ 


$$
\begin{aligned}
& \operatorname{Cov}(\hat{00, \hat{1}})=\operatorname{Cov}(\hat{0} 0, \hat{2})=\operatorname{Cov}(\hat{01}, \hat{0})=\operatorname{Cov}(\hat{0}, \hat{20})= \\
& =\operatorname{cov}\left(\hat{10,2 i)}=\operatorname{cov}(\hat{1}, \hat{20})=-\frac{13}{720} \delta^{2}=-0,0180555 \delta^{2}\right.
\end{aligned}
$$

\subsubsection{Variâncias dos efeitos fatoriais}

Uma vez obtidas as variâncias e covariâncias dos tratamentos é fácil obter as variâncias dos efeitos fatoriais, a seguir determinadas.

a) Variâncias dos efeitos principais

Seja, por exemplo, o fator $A$, cujos níveis' podem assim ser determinados:

$$
\begin{aligned}
& \hat{a}_{0}=\frac{(\hat{00})+(\hat{011})}{2} \\
& \tilde{a}_{1}=\frac{(\hat{10})+(\hat{11})}{2} \\
& \hat{a}_{2}=\frac{(\hat{20)}+(\hat{21)}}{2} \\
& V\left(\hat{a}_{0}\right)=\frac{1}{4}[V(\hat{0} 0)+V(\hat{0})+2 \operatorname{cov}(\hat{0} 0, \hat{0})] \delta^{2} \\
& V(\hat{c})=\frac{1}{4}\left(\frac{269}{720}+\frac{259}{720}-2\left(\frac{109}{720}\right)\right] \delta^{2} \\
& V\left(\hat{a}_{0}\right)=\frac{1}{9} \delta^{2}
\end{aligned}
$$


A mesma variância é obtida para os niveis $\hat{a}_{\mathfrak{l}}$ $\mathrm{e} \hat{a}_{2}$.

Como âo, $\hat{a}_{1}$ e $\hat{a}_{2}$ possuem a mesma variância, então diz-se que a variância do efeito principal $A$ è $\frac{1}{9} \delta^{2}$.

A variância dos niveis de $B$ ë obtida de ma neira anäloga, ou seja:

$$
\begin{aligned}
& \hat{b}_{0}=\frac{(\hat{00})+(\hat{0})+(2 \hat{0})}{3} \\
& \hat{b}_{1}=\frac{(\hat{i})+(\hat{i})+(\hat{i})}{3} \\
& v\left(\hat{b}_{0}\right)=\frac{1}{9}\left[\begin{array}{l}
v(\hat{00})+v(\hat{0})+v(\hat{20})+2 \operatorname{cov}(\hat{00,} \hat{0})+2 \operatorname{cov}(\hat{00}, \hat{20})+2 \operatorname{cov}(\hat{10}, \hat{0}) \\
+2
\end{array}\right] \delta^{2} \\
& V\left(\hat{b}_{0}\right)=\frac{1}{9}\left[3\left(\frac{269}{720}\right)-6\left(\frac{67}{720}\right)\right] \delta^{2} \\
& v\left(\hat{b}_{0}\right)=\frac{1}{16} \xi^{2}=v\left(\hat{b}_{1}\right) .
\end{aligned}
$$

b) Variância das interações

Para se obter a variancia de uma interação de primeira ordem, deve-se primeiramente calcular as variancias dos niveis dos fatores que compõem csta interação, subtraindo-se as respectivas variancias dos niveis dos efeitos princlpais. Assim, para o exemplo, tem-se: 


$$
\begin{aligned}
& V\left(\hat{a}_{0}\right)=V\left(\hat{0}_{0}\right)-V\left(\hat{a}_{0}\right)-V\left(\hat{b}_{0}\right) \\
& V\left(\hat{a}_{0}\right)=(269 / 720-1 / 9-1 / 16) \delta^{2} \\
& v\left(\hat{a}_{0}\right)=1 / 5 \delta^{2}
\end{aligned}
$$

Obtëm-se a mesma variância para âbol, $\hat{a b}_{10}$, $\hat{a}_{13}, \hat{a}_{20}$ e $\hat{a b}_{21}$. Diz-se então que a variância da interação $A B$ è $1 / 5 \delta^{2} e$, sendo a mesma para todos os niveis, o fatorial è dito balanceado nesta interação.

No exemplo citado, as variâncias dos níveis' de $A B$, coincidem com as dos tratamentos, sendo tomadas dire tamente da matriz de dispersão. No caso do fatorial possuir mais de dois fatores, deve-se primeiramente calcular a va riância dos niveis que compõem a interação dupla. Apresenta - se, a seguir, o cálculo da variância de aboo, da interação' $A B$, de um fatorial $3 \times 2^{3}$.

$$
a_{0} \hat{b}_{0}=\frac{(0 \hat{000})+(0 \hat{001})+(0 \hat{10})+(0 \hat{011})}{4}
$$

$$
\begin{aligned}
& v\left(a_{0} \hat{b}_{0}\right)=\frac{1}{16}\left[\begin{array}{l}
v(0 \hat{000})+\ldots+v(0 \hat{011})+2 \operatorname{cov}(0 \hat{000,0001)} \\
+\ldots+2 \operatorname{cov}(0 \hat{010}, 0 \hat{011})
\end{array}\right] \delta^{2} \\
& V\left(\hat{a}_{b} \dot{b}_{0}\right)=V\left(\hat{a}_{0} \hat{b}_{0}\right)-V\left(\hat{a}_{0}\right)-V\left(\hat{b}_{0}\right) \\
& \text { Jä no caso de uma interação de três fatores, }
\end{aligned}
$$
deve-se obter, primeiramente, a variância dos três niveis ' 
que compõem cada combinação desta interação; logo após, subtrair todas as variâncias dos niveis respectivos das intera ções duplas e somar às dos respectivos efeitos principais.

Assim, por exemplo, a variância de a b́booo da interação $A B C$ do fatorial $3 \times 2^{3}$ é obtida da seguinte for ma :

$$
a_{0} \hat{b}_{0} c_{0}=\frac{(00 \hat{0} 0)+(0 \hat{001)}}{2}
$$

$$
\begin{aligned}
& v\left(a_{0} \hat{b}_{0} c_{0}\right)=\frac{1}{4}[v(0 \hat{000})+v(0 \hat{001})+2 \operatorname{cov}(0 \hat{000}, 0 \hat{001})] \\
& V\left(a \hat{b} c_{0} 00\right)=V\left(a_{0} \hat{b}_{0} c_{0}\right)-V\left(a \hat{b}_{0} 0\right)-V\left(\vec{a} \hat{c}_{00}\right)-V\left(\hat{b}_{0}\right)+ \\
& +V\left(\hat{a}_{0}\right)+V\left(\hat{b}_{0}\right)+V\left(\hat{c}_{0}\right)
\end{aligned}
$$

Generalizando o processo de obtenção da va riância de uma combinação dos níveis de uma interação, proaede-se da seguinte forma:

a) Determina-se a variância dessa combinação;

b) Se a interação tem um nümero par de fatores, somam-se todas as variâncias dos respectivos niveis das interações, de menor ordem, com nümero par de fatores e subtrai-se' àquelas com nümero impar, inclusive os efeitos principais respectivos;

c) Se a interação possuir nümero impar te fatores, somam-se as variancias dos respectivos niveis dos efeitos principais e das outras interaçöes, tambèm compostas por um nümero impar de fatores. A seguir subtrai-se as variâncias 
dos niveis respectivos das interações com nümero par de fatores. Naturalmente, todas as interações devem ser de menor ordem daquela que se deseja determinar a varlancia.

c) obtenção da informação relativa

Para a obtenção da informação relativa deve- se, primeiramente, calcular as variàncias dos efeitos fatori ais não considerando o confundimento do fatorial.

Tomando-se como exemplo o fatorial $3 \times 2, \mathrm{com}$ pore-se a matriz C, como se segue:

$$
\begin{gathered}
c_{i j}=(1-1 / 6)(3)=5 / 2 \\
c_{i j}=-3 / 6=-1 / 2 \\
C=\frac{1}{2}\left[\begin{array}{rrrrrr}
5 & -1 & -1 & -1 & -1 & -1 \\
-1 & 5 & -1 & -1 & -1 & -1 \\
-1 & -1 & 5 & -1 & -1 & -1 \\
-1 & -1 & -1 & 5 & -1 & -1 \\
-1 & -1 & -1 & -1 & 5 & -1 \\
-1 & -1 & -1 & -1 & -1 & 5
\end{array}\right]
\end{gathered}
$$

Aplicando-se a restrição:

$$
\frac{1}{2}[(\hat{n})+(\hat{n})+(\hat{n})+(\hat{1})+(\hat{n})+(\hat{i})]=0
$$

obtëm-se a matriz M, diagonal, ou seja: 


$$
M=318
$$

A inversa de $M$ ë:

$$
M^{-1}=\frac{1}{3} I_{6}
$$

e a matriz de oispersão tem a seguinte expressão:

$$
0=\frac{1}{9} c \delta^{2}
$$

Calculando-se as variâncias dos efeitos fato riais,de acordo com o processo anteriormente descrito, obtèm-se:

$$
\begin{aligned}
& V\left(\hat{a}_{0}\right)=\frac{1}{4}\left[V\left(\hat{0}_{0}\right)+V(\hat{0} \hat{1})+2 \operatorname{cov}\left(\hat{0}_{0}, \hat{0}_{1}\right)\right] \\
& V\left(\hat{a}_{0}\right)=\frac{1}{4}\left[\frac{5}{18}+\frac{5}{18}-2\left(\frac{1}{18}\right)\right] \delta^{2} \\
& V\left(\hat{a}_{0}\right)=\frac{1}{9} \delta^{2}=V\left(\hat{a}_{1}\right)=V\left(\hat{a}_{2}\right)
\end{aligned}
$$

Para o efeito principal n, tem-se:

$$
\begin{aligned}
& v(\hat{b} 0)=\frac{1}{9}\left\{\begin{array}{l}
v(\hat{i n})+v(\hat{i n})+v(2 \hat{0})+2 \operatorname{cov}(\hat{00,}, \hat{0})+ \\
+2 \operatorname{cov}(\hat{00,} \hat{0})+2 \operatorname{cov}(\hat{10,} \hat{0})
\end{array}\right\} \\
& V\left(\hat{b}_{0}\right)=\frac{1}{9}\left[3\left(\frac{5}{18}\right)-6\left(\frac{1}{18}\right)\right] \delta^{2} \\
& v\left(\hat{b}_{0}\right)=\frac{1}{T_{8}} \cdot v\left(\hat{b}_{1}\right)
\end{aligned}
$$

Para a interação Af, tem-se:

$$
v\left(\hat{a} \hat{b}_{0} 0\right)=v(\hat{D O})-v\left(\hat{a}_{0}\right)-v\left(\hat{b}_{0}\right)
$$




$$
\begin{aligned}
& V\left(\hat{a}_{0}\right)=(5 / 18-1 / 9-1 / 18) \delta^{2} \\
& V\left(\hat{a}_{0}\right)=1 / 9 \delta^{2}
\end{aligned}
$$

0 mesmo resultado é obtido para ábol, $\hat{a b}_{10}$. $\hat{a}_{11}, \hat{a}_{b_{2}} e \hat{a} \hat{b}_{21}$.

Finalmente, calcula-se a informação relativa atravēs da razão:

$$
\text { Informação relativa }=\frac{\text { Variância Sem Confundimento }}{\text { Variância Com Confundimento }}
$$

cujos resultados para os efeltos fatoriais $A, B$ e $A B$ são mostrados no quadro dado a seguir:

\begin{tabular}{|c|c|c|c|}
\hline Efeitos Fatoriais & V(Sem Confund.) & $V($ Com Confund.) & Inf.Rel. \\
\hline$A$ & $1 / 9 \delta^{2}$ & $1 / 9 \delta^{2}$ & 1 \\
$B$ & $1 / 18 \delta^{2}$ & $1 / 16 \delta^{2}$ & $8 / 9$ \\
$A B$ & $1 / 9 \delta^{2}$ & $1 / 5 \delta^{2}$ & $5 / 9$ \\
\hline
\end{tabular}


4.7. Anālise de variância

Apresentar-se-á apenas o processo geral de obtenção das somas de quadrados dos efeitos fatoriais con fundidos, uma vez que as somas de quadrados daqueles efei tos isentos de confundimento são obtidas da maneira usual. A partir do processo geral é possivel obter uma förmula sim plificada para cada soma de quadrados dos efeitos fatoriais confundidos.

Seja, por exemplo, o fatorial $3 \times 2$ em blocos de três unidades, onde estão confundidos os efeitos fatoriais $B \subseteq A B$.

Como se sabe, as estimativas dos efeitos de tratamentos são dadas por:

$$
\hat{T}=M^{-1} Q
$$

e a soma de quadrados de tratamentos ajustados, por:

$$
\text { s.Q.Trat.aj. }=\hat{T}^{\prime} Q=Q^{\prime} M^{\prime 1} Q=\sum_{i}^{v} \hat{t}_{i} Q_{i} \text {, }
$$

que, no exemplo citado è:

$$
\text { S.Q.Trat.aj. }=(\hat{0} 0) 0.00+(\hat{01}) Q_{01}+\ldots+(\hat{i 1}) Q_{21}
$$

A soma de quadrados do efeito principal B ë 
obtida calculando-se,primeiramente, os efeitos de tratamentos $\hat{b}_{0}$ e $\hat{b}_{1}$ da seguinte forma:

$$
\begin{aligned}
& \hat{b}_{0}=\frac{(\hat{00})+(\hat{0})+(\hat{00})}{3} \\
& \hat{b}_{1}=\frac{(\hat{0} 1)+(\hat{1})+(\hat{2})}{3}
\end{aligned}
$$

Após, determinam-se os Q's correspondentes, ou sejam :

$$
\begin{aligned}
& Q_{0}=Q_{00}+Q_{10}+Q_{20} \\
& Q_{1}=Q_{01}+Q_{11}+Q_{21}
\end{aligned}
$$

Assim procedendo, obtém-se:

$$
S \cdot Q \cdot B=\hat{b}_{0} Q_{0}+\hat{t}_{1} Q_{1}
$$

A S.Q. da interação $A B$ é dada por:

$$
\text { S.Q.AB = S.Q.Trat.aj. - S.Q.A-S.Q.B }
$$

No caso da soma de quadrados de uma interação de très fatores, $A B C$, por exemplo, determina-se primeiramente $\circ \sum \hat{t}_{i}{ }^{0} \mathbf{i}$ pelo processo descrito e após subtrai-se as somas de quadrados das interações $A B, A C$ e $B C$ e as somas de quadra dos dos efeitos principais $A, B \in C$.

Raciocinio anālogo é aplicado às interações ' com mais fatores.

Como no presente trabalho a preocupação ë a 
obtenção e anälise de fatoriais balanceados, a decomposição dos graus de liberdade de tratamentos, para os efeitos fato riais, nesses casos, não oferece problema, uma vez que não hä perda dos mesmos nas interações parcialmente confundidas. Assim, por exemplo, para o conjunto balanceado, composto de quatro repetições, do fatorial $3^{3}$, em blocos de nove unidades, tem-se o seguinte esquema de anälise:

\begin{tabular}{c|c}
\hline Causas da Variação & G.L. \\
\hline Blocos & 11 \\
$A$ & 2 \\
$B$ & 2 \\
$C$ & 2 \\
$A B$ & 4 \\
$A C$ & 4 \\
$B C$ & 4 \\
ABC & 8 \\
Residuo & 70 \\
\hline Total & 107 \\
\hline
\end{tabular}

Pode-se verificar que, em cada repetição,confunde-se dois graus de liberdade da interação $A B C$. Entretanto, estes são recuperados nas outras três, de forma que não hă perda dos mesmos. Ocorre apenas perda da informação oride os dois graus de liberdade estão confundidos. 
Por outro lado, usando-se apenas uma repetição do referido fatorial, tem-se

\begin{tabular}{c|c}
\hline Causas da Variação & G.L. \\
\hline Blocos & 2 \\
A & 2 \\
$B$ & 2 \\
C & 2 \\
AR & 4 \\
Ar. & 4 \\
Br. & 4 \\
ARC (Residuo) & 6 \\
\hline Total & 26 \\
\hline
\end{tabular}

isto $:$ os dois graus de liherdade de blocos são provenientes da interação $A B C$. Não havendo outras repetições, a inte ração $A B C$ perde esses graus de liberdade, os quais flcam' confundidos com efeitos de blocs. Nesse fatorial sem repetição, a parte da interação $A B C$ que não fica confundida é utllizada para residuo.

Tambēm ocorrerä a perda de graus de liberdade se o mesmo confundimento for empregado em todas as repetições. E o que ocorre, por exemplo, na subdivisão em dois blocos dos fatoriais da sērie $2^{n}$, quando se confunde inte ração de mais alta ordem. 


\section{RESULTADOS}

A seguir, são dados os planos dos fatoriais balanceados obtidos de acordo com a metodologia anterior mente descrita, bem como as interações confundidas e sua respectiva informação relativa.

5.1. Fatoriais balanceados da série $2^{\text {n }}$

5.1.1. Fatorial 22

Blocos de duas unidades

Interação $A B$ completamente confundida Nümero de repetições: duas ou mais. 


\begin{tabular}{|c|c|c|}
\cline { 2 - 3 } \multicolumn{1}{c|}{} & \multicolumn{2}{|c|}{ REPETICÃ 1} \\
\hline Rlocos & 1 & 2 \\
\hline & $(1)$ & $a$ \\
& $a h$ & $b$ \\
\hline
\end{tabular}

5.1.2. Fatorial $2^{3}$

a) Blocos de quatro unidades

Interação $A B C$ completamente confundida Nümero de repetiçöas: duas ou mais.

\begin{tabular}{|c|c|c|}
\hline \multicolumn{1}{c|}{} & \multicolumn{2}{|c|}{ REPET ICÄO I } \\
\hline Blocos & 1 & 2 \\
\hline & $a$ & $(1)$ \\
$b$ & $a b$ \\
$c$ & $a c$ \\
ahc & $b c$ \\
\hline
\end{tabular}

b) Blocos de duas unidades

Interações $A B$, $A C, e B C$ completamente confundidas.

Nümero de repetições: duas ou mais. 


\begin{tabular}{|c|c|c|c|c|}
\hline \multirow[b]{2}{*}{ Blocos } & \multicolumn{4}{|c|}{ REPET IÇÃO 1.} \\
\hline & 1 & 2 & 3 & 4 \\
\hline & (1) & $a$ & $b$ & $c$ \\
\hline & abc & bc & $a c$ & $a b$ \\
\hline
\end{tabular}

5.1.3. Fatortal $2^{4}$

a) Blocos de "to unidades

\begin{tabular}{|c|c|c|}
\hline & REPETI & $\bar{A} 0 \quad 1$ \\
\hline \multirow[t]{9}{*}{ Rlocos } & 1 & 2 \\
\hline & (1) & $a$ \\
\hline & $a b$ & b \\
\hline & $a c$ & c \\
\hline & ad & $d$ \\
\hline & $b c$ & $a b c$ \\
\hline & hd & $a b d$ \\
\hline & $c d$ & acd \\
\hline & abcd & $b c d$ \\
\hline
\end{tabular}

b) Blocos de quatro unidades

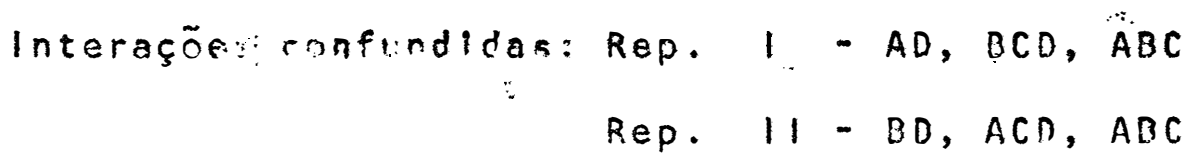


Interaçöes confundidas: Rep. $|1|-C D, A B D, A B C$

Rep. IV - $A C, B C D, A B D$

Rep. $V-B C, A C D, A B D$

Rep. $V I-A B, B C D, A C D$

Informação relativa: $A B, A C, A D, B C, B \cap, C D: 5 / 6$

$A B C, A B D, A C D, B C D: 1 / 2$

\begin{tabular}{|c|c|c|c|c|c|c|c|c|}
\hline & \multicolumn{3}{|c|}{ REPETICÃO } & \multirow{2}{*}{4} & \multicolumn{4}{|c|}{ REPETICÃO } \\
\hline Blocos & 1 & 2 & 3 & & 1 & 2 & 3 & 4 \\
\hline & $b$ & (1) & $a b$ & $a$ & a & (1) & $a b$ & $b$ \\
\hline & $c$ & bc & ac & $a b c$ & c & ac & bc & $a b c$ \\
\hline & ad & abd & d & $b d$ & $b d$ & $a b d$ & $d$ & ad \\
\hline & abed & & & cd & & bcd & acd & $c d$ \\
\hline
\end{tabular}

\begin{tabular}{|r|c|c|c|c||c|c|c|c|}
\hline \multicolumn{1}{c|}{} & \multicolumn{4}{|c|}{ REPETICÃO 111} & \multicolumn{4}{|c|}{ REPETICÃO IV } \\
\hline Blocos & 1 & 2 & 3 & 4 & 1 & 2 & 3 & 4 \\
\hline & $a$ & $(1)$ & $c$ & $a c$ & $b$ & $(1)$ & $a b$ & $a$ \\
$b$ & $a b$ & $a b c$ & $b c$ & $d$ & $b d$ & $a d$ & $a b d$ \\
$c d$ & $a c d$ & $a d$ & $d$ & $a c$ & $a b c$ & $c$ & $b c$ \\
$a b c d$ & $b c d$ & $b d$ & $a b d$ & $a b c d$ & $a c d$ & $b c d$ & $c d$ \\
\hline
\end{tabular}




\begin{tabular}{|c|c|c|c|c|c|c|c|c|}
\hline \multirow[b]{2}{*}{ Blocos } & \multicolumn{4}{|c|}{ REPETICÃO V } & \multicolumn{4}{|c|}{ REPETICÃO VI } \\
\hline & 1 & 2 & 3 & 4 & 1 & 2 & 3 & 4 \\
\hline & a & (1) & $a b$ & $b$ & c & (1) & a & ac \\
\hline & $d$ & ad & $h d$ & $a b d$ & $d$ & $c d$ & acd & ad \\
\hline & $b c$ & $a b c$ & c & ac & $a b$ & $a b c$ & $b c$ & $b$ \\
\hline & abed & bcd & acd & $c d$ & abod & $a b d$ & $b d$ & bed \\
\hline
\end{tabular}

c) Blocos die duas unidades

Interações $A \cap, A C, A \cap, B C, B n, C D$ e ABCD completamente confundidas .

Nümero de repetições: duas ou mais.

\begin{tabular}{|c|c|c|c|c|c|c|c|c|}
\hline & \multicolumn{8}{|c|}{ REPETIRÃO I } \\
\hline \multirow[t]{3}{*}{ Blocos } & 1 & 2. & 3 & 4 & 5 & 6 & 7 & 8 \\
\hline & (1) & a & b & c & $d$ & $a b$ & $a c$ & ad \\
\hline & $a b c d$ & $b c d$ & $\mathrm{acd}$ & ahd & $a b c$ & $c d$ & bd & $b c$ \\
\hline
\end{tabular}

5.1.4. Fatorial $2^{5}$

a) Blocos de dezesseis unidades

Interação ABCnE completamente confundida. Nümero de repetições: duas ou mais. 


\begin{tabular}{|c|c|c|}
\hline & REPET & Ão I \\
\hline \multirow[t]{17}{*}{$810 \cos$} & 1 & 2 \\
\hline & $a$ & (1) \\
\hline & $b$ & $a b$ \\
\hline & $c$ & ac \\
\hline & $d$ & ad \\
\hline & $e$ & ae \\
\hline & abc & bc \\
\hline & $a b d$ & $b d$ \\
\hline & abe & be \\
\hline & acd & $c d$ \\
\hline & ace & $\mathrm{ce}$ \\
\hline & ade & de \\
\hline & bcd & abcd \\
\hline & hce & abce \\
\hline & bde & abde \\
\hline & cde & acde \\
\hline & abcde & bcde \\
\hline
\end{tabular}

b) Blocos de oito unidades

Interações confundidas: Rep. 1 - $A B E, C D E, A B C D$

Rep. $\quad 11-A C D, B D E, A B C E$ 
Interações confundidas: Rep. III - ACE, BCn, ABחE ReD. IV - ABD, BCE, ACDE Ren. $V$ - ABC, $A D E, B C D E$ Informação relativa: $A B r, A B D, A B E, A C D, A C E, A D E$, DCD, BCE, BDE, COE, $A B C D$, ABCE, ASTE, ACDE, BCDE: 4/5

\begin{tabular}{|c|c|c|c|c|c|c|c|c|}
\hline \multirow[b]{2}{*}{ Blocos } & \multicolumn{4}{|c|}{ REPETISÃO I } & \multicolumn{4}{|c|}{ REPETICTO II } \\
\hline & 1 & 2 & 3 & $i$ & $!$ & 2 & 3 & 4 \\
\hline & $A C$ & a & c & (1) & 05 & a & $y$ & (1) \\
\hline & ad & $\mathrm{acd}$ & 4 & $\mathrm{CH}$ & ao & abe & $e$ & be \\
\hline & $b c$ & $b$ & $a b c$ & $2 b$ & $b c$ & c & $a b c$ & $a c$ \\
\hline & bd & bod & abd & abcd & $\mathrm{ce}$ & $b c e$ & ace & $a b c e$ \\
\hline & $\epsilon$ & ce & ae & $a c e$ & $d$ & bd & ad & $a b d$ \\
\hline & cde & de & acde & acio & bde & de & $a b d e$ & ade \\
\hline & abe & abce & he & $b c e$ & $\operatorname{eod}$ & abcd & $c d$ & bed \\
\hline & abcde & abde & bode & $b$ be & abodo & acde & bcde & $c d e$ \\
\hline
\end{tabular}

\begin{tabular}{|c|c|c|c|c|c|c|c|c|}
\hline & \multicolumn{4}{|c|}{ REPETICEAO $\| 1$} & \multicolumn{4}{|c|}{ REPETIR,TO IV } \\
\hline Blocos & 1 & 2 & 3 & 4 & 1 & 2 & 3 & 4 \\
\hline & $a b$ & a & b & (1) & $a c$ & $a$ & c & (1) \\
\hline & ad & abd & $d$ & hd & ae & ace & e & $\mathrm{ce}$ \\
\hline & c & $b c$ & $a c$ & $a h c$ & $b$ & $b c$ & $a b$ & $a b c$ \\
\hline & bcd & $c d$ & abcd & act & bce & $b$ & $a b c c$ & abe \\
\hline & be & e & abe & ae & cd & d & acd & ad \\
\hline & de & bde & ade & abde & de & cde & ade & $\operatorname{acde}$ \\
\hline & ace & abce & $\mathrm{ce}$ & bce & abd & abcd & $b d$ & bed \\
\hline & abcde & acde & bcde & cde & llabede & abde & bcde & bde \\
\hline
\end{tabular}


105.

\begin{tabular}{|c|c|c|c|c|}
\hline \multicolumn{1}{c|}{} & \multicolumn{4}{|c|}{ REPETICÃo V } \\
\hline Blocos & 1 & 2 & 3 & 4 \\
\hline \multirow{4}{*}{} & $a$ & $a d$ & $d$ & $(1)$ \\
ade & ae & $e$ & $d e$ \\
bd & $b$ & ab & abd \\
he & bde & abde & abe \\
$c d$ & $c$ & ac & acd \\
$c e$ & $c d e$ & acde & ace \\
& abc & abcd & bcd & bc \\
abcde & abce & bce & bcde \\
\hline
\end{tabular}

c) Blocos de quatro unidades

Interações confundidas: Rep. I - An, BE, $A B C, A C E, B C D$, CDE, $A B D E$

Rep. $\quad 11-A C, D E, A B D, A B E, B C D$, $B C E, A C D E$

Rep. $\| 11-B D, C E, A B C, A B E, A C D$, $A D E, B C D E$

Ren. IV - $A B, C D, A C E, A D E, B C E$, $B D E, A B C D$

Rep. $V-A E, B C, A B D, A C D, B D E$, $C D E, A B C E$

Informação relativa: $A B, A C, A \cap, A E, B C, B D, B E, C D, C E$, DF: $4 / 5$ 
Informação relativa: $A B C, A B \cap, A B E, A C D, A C E, A D E, B C D$,

BCE, BDE, CDE: $3 / 5$

ABCD, ARC,, ABDE, ACDE, RCDE:4/5

\begin{tabular}{|c|c|c|c|c|c|c|c|c|}
\hline \multicolumn{1}{|c|}{} & \multicolumn{8}{|c|}{ REPETI C.̃̃o } \\
\hline Blocos & 1 & 2 & 3 & 4 & 5 & 6 & 7 & 8 \\
\hline & ad & a & ade & ae & de & $e$ & $d$ & $(1)$ \\
& be & bde & $b$ & bd & ab & abd & abe & abde \\
& $c$ & $c d$ & $c e$ & $c d e$ & ace & acde & ac & acd \\
& abcde & abce & abcd & abc & bcd & bc & bcde & bce \\
\hline
\end{tabular}

\begin{tabular}{|c|c|c|c|c|c|c|c|c|}
\hline Blocos & \multicolumn{8}{|c|}{ REPETICÃO $\|$} \\
\hline Blocos & 1 & 2 & 3 & 4 & 5 & 6 & 7 & 8 \\
\hline & $a c$ & a & ace & ae & $\mathrm{ce}$ & e & c & (1) \\
\hline & $b$ & $b c$ & be & bce & abe & abce & $a b$ & $a b c$ \\
\hline & de & cde & d & $c d$ & ad & acd & ade & acde \\
\hline & abcde & abde & $a b c d$ & $a b d$ & $\operatorname{bed}$ & bd & bcde & bde \\
\hline
\end{tabular}

\begin{tabular}{|c|c|c|c|c|c|c|c|c|}
\hline & \multicolumn{8}{|c|}{ REPETIC $\bar{A} O\|\|$} \\
\hline \multirow[t]{5}{*}{$310 \mathrm{cos}$} & 1 & 2 & 3 & 4 & 5 & 6 & 7 & 8 \\
\hline & a & ad & ade & ae & e & de & $d$ & (1) \\
\hline & $b d$ & b & be & bde & abde & abe & $a b$ & abd \\
\hline & ce & cde & $c d$ & c & $a c$ & acd & acde & ace \\
\hline & abcde & abce & $a b c$ & abcd & $b c d$ & $b c$ & bce & bcde \\
\hline
\end{tabular}




\begin{tabular}{|c|c|c|c|c|c|c|c|c|}
\hline & \multicolumn{8}{|c|}{ REPETICQĀO IV } \\
\hline Blocos & 1 & 2 & 3 & 4 & 5 & 6 & 7 & 8 \\
\hline & $a b$ & a & $a b d$ & ad & bd & d & $b$ & $(1)$ \\
\hline & $c d$ & bcd & c & bc & ac & abc & acd & abcd \\
\hline & e & be & de & bde & ade & abde & ae & a be \\
\hline & abcde & acde & abce & ace & bce & ce & bcde & cde \\
\hline
\end{tabular}

\begin{tabular}{|c|c|c|c|c|c|c|c|c|}
\hline & \multicolumn{8}{|c|}{ REPETIC, AO V } \\
\hline \multirow[t]{5}{*}{ Blocos } & 1 & 2 & 3 & 4 & 5 & 6 & 7 & 8 \\
\hline & ae & a & ace & $a c$ & ce & $c$ & e & (1) \\
\hline & $b c$ & hce & $b$ & be & $a b$ & $a b e$ & $a b c$ & abce \\
\hline & $d$ & $d e$ & $c d$ & cde & acd & acde & $a d$ & ade \\
\hline & abcde & abcd & abde & abd & bde & $b d$ & bode & bed \\
\hline
\end{tabular}

d) Blocos de duas unidades

Interações completamente confundidas: $A B, A C, A D, A E, B C$, BD, BE, CD, CE, DE, $A B C D, A B C E, A B D E$, $A C D E$, BCDE.

Nümero de repetições: duas ou mais. 


\begin{tabular}{|c|c|c|c|c|c|c|c|c|}
\hline & \multicolumn{8}{|c|}{ REPETICÃO I } \\
\hline Blocos & 1 & 2 & 3 & 4 & 5 & 6 & 7 & 8 \\
\hline & $\begin{array}{c}(1) \\
\text { abcde }\end{array}$ & bede & $\begin{array}{c}b \\
\text { acde }\end{array}$ & $\begin{array}{c}c \\
\text { abde }\end{array}$ & $\begin{array}{c}d \\
a b c e\end{array}$ & $\begin{array}{c}e \\
a b c d\end{array}$ & $\begin{array}{l}a b \\
c d e\end{array}$ & $\begin{array}{r}a c \\
b d e\end{array}$ \\
\hline Blocos & 9 & 10 & וז & 12 & 13 & 14 & 15 & 16 \\
\hline & $\begin{array}{l}\text { ad } \\
\text { bce }\end{array}$ & $\begin{array}{l}\text { ae } \\
\text { bcd }\end{array}$ & $\begin{array}{l}\text { bc } \\
\text { ade }\end{array}$ & $\begin{array}{l}\text { bd } \\
\text { ace }\end{array}$ & $\begin{array}{l}\text { be } \\
\text { acd }\end{array}$ & $\begin{array}{c}c d \\
a b e\end{array}$ & $\begin{array}{l}c e \\
\text { abd }\end{array}$ & $\begin{array}{l}\text { de } \\
a b c\end{array}$ \\
\hline
\end{tabular}

5.2. Fatoriais balanceados da série $3^{\text {n }}$

$$
\text { 5.2.1. Fatorial } 3^{2}
$$

Blocos de três unidades

Interação confundida: $A B$

Informação relativa: $1 / 2$

\begin{tabular}{|c|c|c|c|}
\hline & \multicolumn{2}{|c|}{ REPET. } & 1 \\
\hline Blocos & 1 & 2 & 3 \\
\hline & 20 & 21 & 22 \\
\hline & 11 & 12 & 10 \\
\hline & 02 & 00 & 01 \\
\hline
\end{tabular}

\begin{tabular}{|l|l|l|l|}
\cline { 2 - 4 } \multicolumn{1}{c|}{} & \multicolumn{3}{|c|}{ REPET. 11} \\
\hline Blocos & 1 & 2 & 3 \\
\hline & 20 & 21 & 22 \\
12 & 10 & 11 \\
01 & 02 & 00 \\
\hline
\end{tabular}


5.2.2. Fatorial $3^{3}$

a) Rlocos de nove unidades

Interação confundida: $A B C$

Informação relativa: $3 / 4$

\begin{tabular}{|c|c|c|c|}
\cline { 2 - 4 } \multicolumn{1}{c|}{} & \multicolumn{3}{|c|}{ REPETICÃO 1} \\
\hline Blocos & 1 & 2 & 3 \\
\hline \multirow{10}{*}{} & 200 & 201 & 202 \\
110 & 111 & 112 \\
020 & 021 & 022 \\
211 & 212 & 210 \\
121 & 122 & 120 \\
& 001 & $0 n 2$ & 000 \\
& 222 & 220 & 221 \\
102 & 100 & 101 \\
012 & 010 & 011 \\
\hline
\end{tabular}

\begin{tabular}{|c|c|c|c|}
\hline & \multicolumn{2}{|c|}{ REPETIÇÃO } & 11 \\
\hline Blocos & 1 & 2 & 3 \\
\hline & 200 & 201 & 202 \\
\hline & 110 & 191 & N12 \\
\hline & 020 & 021 & 022 \\
\hline & 212 & 210 & 211 \\
\hline & 122 & 120 & 121 \\
\hline & 002 & 000 & 001 \\
\hline & 221 & 222 & 220 \\
\hline & 101 & 102 & 100 \\
\hline & 011 & 012 & 010 \\
\hline
\end{tabular}

\begin{tabular}{|c|c|c|c|}
\hline \multicolumn{1}{c|}{} & \multicolumn{3}{|c|}{ REFETICAOC 11} \\
\hline BIOCOS & 1 & 2 & 3 \\
\hline & 200 & 201 & 202 \\
120 & 121 & 122 \\
$n 10$ & 011 & $n 12$ \\
211 & 212 & 210 \\
101 & 122 & 100 \\
$n 21$ & $n 22$ & $n 20$ \\
222 & 220 & 221 \\
112 & 110 & 111 \\
$n n 2$ & $n n n$ & $n n 1$ \\
\hline
\end{tabular}

\begin{tabular}{|c|c|c|c|}
\hline \multirow[b]{2}{*}{ Blocos } & \multicolumn{2}{|c|}{ REPETICÃO } & \multirow{2}{*}{$\frac{\text { IV }}{3}$} \\
\hline & 1 & 2 & \\
\hline & 200 & 201 & 202 \\
\hline & 120 & 121 & 122 \\
\hline & 010 & 011 & 012 \\
\hline & 212 & 210 & 211 \\
\hline & 132 & $10 n$ & 101 \\
\hline & 022 & $02 n$ & 021 \\
\hline & 221 & 222 & 220 \\
\hline & 111 & 112 & 110 \\
\hline & 001 & 002 & $0 \cap 0$ \\
\hline
\end{tabular}


b) Blocos de três unidades

Interações confundidas: $A B, A C, B C, A B C$.

Informação relativa: $A B, A C, B C: 1 / 2$

$A B C: 3 / 4$

\begin{tabular}{|c|c|c|c|c|c|c|c|c|c|}
\hline & \multicolumn{9}{|c|}{ REPETI६ÃO । } \\
\hline Blocos & 1 & 2 & 3 & 4 & 5 & 6 & 7 & 8 & 9 \\
\hline & $2 \cap 0$ & 201 & 272 & 210 & 211 & 212 & 220 & 221 & 222 \\
\hline & 111 & 112 & 110 & 121 & 122 & 120 & 101 & 102 & 100 \\
\hline & 022 & 020 & 021 & $0 \cap 2$ & $0 \cap 0$ & 001 & 012 & חוּח & 011 \\
\hline
\end{tabular}

\begin{tabular}{|c|c|c|c|c|c|c|c|c|c|}
\hline \multirow[b]{2}{*}{ Blocos } & \multicolumn{9}{|c|}{ REPETIÇÃO } \\
\hline & 1 & 2 & 3 & 4 & 5 & 6 & 7 & 8 & 9 \\
\hline & 200 & 201 & 202 & 210 & 211 & 212 & 220 & 221 & 222 \\
\hline & 112 & $\ln$ & 111 & 122 & 120 & 121 & 102 & $\ln n$ & 101 \\
\hline & 021 & 022 & $72 n$ & וnח & 002 & $00 n$ & 011 & 012 & 010 \\
\hline
\end{tabular}

\begin{tabular}{|c|c|c|c|c|c|c|c|c|c|}
\hline \multicolumn{1}{|c|}{} & \multicolumn{8}{|c|}{ REPETICAO 111} \\
\hline B10cos & 1 & 2 & 3 & 4 & 5 & 6 & 7 & 8 & 9 \\
\hline & 200 & $2 n 1$ & $2 n 2$ & 210 & 211 & 212 & 220 & 221 & 222 \\
121 & 122 & $12 n$ & 101 & 102 & 100 & 111 & 112 & 110 \\
$n 12$ & 010 & $n 11$ & $n 22$ & $n 20$ & $n 21$ & $0 n 2$ & $n n 0$ & $n n 1$ \\
\hline
\end{tabular}




\begin{tabular}{|c|c|c|c|c|c|c|c|c|c|}
\hline \multirow[b]{2}{*}{ Blocos } & \multicolumn{9}{|c|}{ REPET IÇÃO } \\
\hline & 1 & 2 & 3 & 4 & 5 & 6 & 7 & 8 & 9 \\
\hline & 200 & 201 & $2 \cap 2$ & 2.10 & 211 & 212 & 220 & 221 & 222 \\
\hline & 122 & 120 & 121 & 102 & $10 n$ & 181 & 112 & 110 & 111 \\
\hline & 011 & 012 & 010 & 021 & 022 & 020 & 001 & $\cap \cap 2$ & $0 \cap 0$ \\
\hline
\end{tabular}

$5 \cdot 2 \cdot 3$. Fatorial $3^{4}$

a) Blocos de vinte e sete unidades

Interação confundida: $A B C D$

Informação relativa: $7 / 8$ 


\begin{tabular}{|c|c|c|c|}
\hline & \multicolumn{3}{|c|}{ REFETICAO I } \\
\hline Blocos & 1 & 2 & 3 \\
\hline & 2000 & 2001 & $2 n \cap 2$ \\
\hline & 1100 & $\ln 1$ & $11 \cap 2$ \\
\hline & 0200 & 0201 & $\cap 2 \cap 2$ \\
\hline & 2110 & 2111 & 2112 \\
\hline & $121 n$ & 1211 & 1212 \\
\hline & 0 & $0 \cap 11$ & $n \cap 12$ \\
\hline & 2220 & 2221 & 2222 \\
\hline & 1020 & 1021 & $1 \cap 22$ \\
\hline & 0120 & 0121 & 0122 \\
\hline & 2011 & 2012 & 2010 \\
\hline & 1111 & 1112 & 1110 \\
\hline & 0211 & 0212 & $n 210$ \\
\hline & 2121 & 2122 & 2120 \\
\hline & 1221 & 1222 & $122 \pi$ \\
\hline & 0021 & $n \cap 22$ & 0020 \\
\hline & $22 n 1$ & $22 \cap 2$ & 2200 \\
\hline & $\operatorname{lnn1}$ & 1002 & $10 n \pi$ \\
\hline & 0101 & 0102 & $n 10 n$ \\
\hline & $2 \cap 22$ & $202 \cap$ & $2 \cap 21$ \\
\hline & 1122 & 1123 & 1121 \\
\hline & 0222 & $n 220$ & 0221 \\
\hline & $21 \cap 2$ & $210 n$ & $21 \cap 1$ \\
\hline & 1202 & 1200 & $12 \cap 1$ \\
\hline & 01002 & 0000 & 0001 \\
\hline & 2212 & 2210 & 2211 \\
\hline & 1912 & $\ln 10$ & 1011 \\
\hline & 0112 & 0110 & 0111 \\
\hline
\end{tabular}

\begin{tabular}{|c|c|c|c|}
\hline \multirow[b]{2}{*}{ Blocos } & \multicolumn{2}{|c|}{ REPETICÃO } & \multirow{2}{*}{$\frac{11}{3}$} \\
\hline & 1 & 2 & \\
\hline & $20 \cap 0$ & 2001 & 2002 \\
\hline & 1100 & 1101 & 1102 \\
\hline & $02 \cap 0$ & 0201 & 0202 \\
\hline & 2110 & 2111 & 2112 \\
\hline & 1210 & 1211 & 1212 \\
\hline & $0 n 10$ & $n \cap 11$ & $0 \cap 12$ \\
\hline & $222 n$ & 2221 & 2222 \\
\hline & 1020 & 1021 & 1022 \\
\hline & $n 12 n$ & 0121 & $n 122$ \\
\hline & $2 n 12$ & 2710 & 2011 \\
\hline & 1112 & 1110 & 1111 \\
\hline & 7212 & 0210 & 0211 \\
\hline & 2122 & 2120 & 2121 \\
\hline & 1222 & 1220 & 1221 \\
\hline & $0 \cap 22$ & 0020 & 0021 \\
\hline & 2202 & $220 n$ & $22 n 1$ \\
\hline & 1002 & 1000 & 1001 \\
\hline & 0102 & 0100 & וnוח \\
\hline & $2 \cap 21$ & 2022 & 2020 \\
\hline & 1121 & 1122 & 1120 \\
\hline & 0221 & 0222 & $022 n$ \\
\hline & 2101 & 2102 & 2100 \\
\hline & 120.1 & 1202 & 1200 \\
\hline & 0001 & 0002 & $0 n 00$ \\
\hline & 2211 & 2212 & $221 n$ \\
\hline & 1011 & 1012 & 010 \\
\hline & 0111 & 0112 & ח \\
\hline
\end{tabular}




\begin{tabular}{|c|c|c|c|}
\hline & \multicolumn{2}{|c|}{ REPFTIC:ÃO } & 111 \\
\hline \multirow[t]{28}{*}{ Blocos } & 1 & 2 & 3 \\
\hline & 2000 & 2001 & 2002 \\
\hline & $110 n$ & 1101 & 1102 \\
\hline & 0200 & $02 \cap 1$ & $02 \cap 2$ \\
\hline & 2120 & 212.1 & 2122 \\
\hline & 1220 & 1221 & 1222 \\
\hline & $0 \cap 2 n$ & $n \cap 21$ & $0 \div 22$ \\
\hline & 2210 & 2211 & 2212 \\
\hline & 1010 & 1011 & $\ln 12$ \\
\hline & 0110 & $n 11$ & 0112 \\
\hline & 2011 & $2 n ! 2$ & 2010 \\
\hline & 1111 & 1112 & 1110 \\
\hline & $n 211$ & 0212 & $n 210$ \\
\hline & 2101 & 2132 & $210 n$ \\
\hline & 1201 & 1202 & 1200 \\
\hline & $0 n 01$ & $n \cap \cap 2$ & nกnก \\
\hline & 2221 & 2222 & 2220 \\
\hline & $1 \cap 21$ & 1022 & 1020 \\
\hline & $n 121$ & 0122 & 0120 \\
\hline & 2022 & $2 \cap 20$ & 2021 \\
\hline & 1122 & 1120 & 1121 \\
\hline & 0222 & 0220 & 0221 \\
\hline & 2112 & 2110 & 2111 \\
\hline & 1212 & 1210 & 1211 \\
\hline & 0012 & nnin & 0011 \\
\hline & 2202 & $22 n n$ & $22 n 1$ \\
\hline & 1002 & $\ln 0$ & $\ln n 1$ \\
\hline & $01 \cap 2$ & $010 n$ & 0101 \\
\hline
\end{tabular}

\begin{tabular}{|c|c|c|c|}
\hline & \multicolumn{2}{|c|}{ REPETICAO } & IV \\
\hline Blocos & 1 & 2 & 3 \\
\hline & 2000 & 2001 & 2002 \\
\hline & 1100 & 1101 & 1102 \\
\hline & $02 \cap 0$ & 0201 & 0202 \\
\hline & 2120 & 2121 & 2122 \\
\hline & 1220 & 1221 & 1222 \\
\hline & 0020 & $0 \cap 21$ & 0022 \\
\hline & 2210 & 2211 & 2212 \\
\hline & 1010 & 1011 & 1012 \\
\hline & 0110 & 0111 & 0112 \\
\hline & 2012 & $201 n$ & 2011 \\
\hline & 1112 & 1110 & 1111 \\
\hline & $n 212$ & 0210 & 0211 \\
\hline & 2102 & 2100 & 2101 \\
\hline & 1202 & 1200 & 1201 \\
\hline & $0 \cap 02$ & $0 \cap 00$ & $n \cap 01$ \\
\hline & 2222 & $222 n$ & 2221 \\
\hline & $1 \cap 22$ & $102 n$ & 1021 \\
\hline & 0122 & $012 n$ & 0121 \\
\hline & 2021 & 2722 & 2020 \\
\hline & 1121 & 1122 & $112 n$ \\
\hline & $\cap 221$ & $\cap 222$ & 0220 \\
\hline & 2111 & 2112 & 2117 \\
\hline & 1211 & $\cdot 212$ & $121 n$ \\
\hline & 0011 & $n \cap 12$ & 0010 \\
\hline & 2201 & 2202 & $22 \cap 0$ \\
\hline & 0001 & $\ln \cap 2$ & 1000 \\
\hline & 0101 & 0102 & 0100 \\
\hline
\end{tabular}


114.

\begin{tabular}{|c|c|c|c|c|c|c|c|}
\hline \multirow[b]{2}{*}{ Blocos } & \multicolumn{3}{|c|}{ REPETICÃ $\mathrm{V}$} & \multirow[b]{2}{*}{ Blocos } & \multicolumn{2}{|c|}{ REPETICÃO } & VI \\
\hline & 1 & 2 & 3 & & 1 & 2 & 3 \\
\hline & 2000 & 2001 & 2002 & & 2000 & 2001 & 2002 \\
\hline & 1200 & 1201 & 1202 & & 1200 & $12 n 1$ & 1202 \\
\hline & 0100 & 0101 & ก102 & & חסוח & וח & 0102 \\
\hline & 2110 & 2111 & 2112 & & 2110 & 2111 & 2112 \\
\hline & 1010 & 1011 & $1 \cap 12$ & & $\ln 1 n$ & 1011 & 1012 \\
\hline & $n 21 \pi$ & 0211 & 0212 & & 0210 & 0211 & 0212 \\
\hline & $222 n$ & 2221 & 2222 & & $222 \pi$ & 2221 & 2222 \\
\hline & 1120 & 1121 & 1122 & & 1127 & 1121 & 1122 \\
\hline & $n \cap 2 n$ & $7 \cap 21$ & $n \cap 22$ & & $n 32 n$ & $n \cap 21$ & 0022 \\
\hline & 2011 & $2 \cap 12$ & 2010 & & $2 \cap 12$ & $2 \cap 1 \cap$ & 2011 \\
\hline & 1211 & 1212 & $121 n$ & & 1212 & 1210 & 1211 \\
\hline & 0111 & 0112 & 0110 & & 0112 & 0110 & וו \\
\hline & 2121 & 2122 & 2127 & & 2122 & 2120 & 2121 \\
\hline & $\ln 21$ & 1722 & $\ln 2 n$ & & 1022 & $102 n$ & 1021 \\
\hline & 0221 & 3222 & $n 220$ & & 0222 & 0220 & $n 221$ \\
\hline & 2201 & $22 ? 2$ & $22 \cap n$ & & $22 \cap 2$ & 2200 & 2201 \\
\hline & 1191 & 1102 & $110 n$ & & 1102 & nחו & 1101 \\
\hline & חกח & $n \cap \cap 2$ & חดח & & $\cap \cap \cap 2$ & $0 \cap 00$ & 0001 \\
\hline & 2.022 & $202 n$ & $2 \cap 21$ & & 2021 & $2 \cap 22$ & $2 ? 20$ \\
\hline & 1222 & $122 n$ & 1221 & & 1221 & 1222 & 1220 \\
\hline & 0122 & $n 12 n$ & 0121 & & 0121 & 0122 & 0120 \\
\hline & 2102 & 2100 & 2101 & & 2101 & $21 \cap 2$ & 2100 \\
\hline & $\ln n 2$ & $\ln n n$ & $10 n 1$ & & 1001 & 1002 & 1000 \\
\hline & $\cap 2 \cap 2$ & $n 2 n 0$ & 0201 & & $n 2 n 1$ & 0202 & $n 2 n n$ \\
\hline & 22.12 & 2217 & 2211 & & 2211 & 2212 & 2210 \\
\hline & 1112 & 1110 & 1111 & & 1111 & 1112 & 1110 \\
\hline & $n \cap 12$ & nחוn & $3 \cap 11$ & & II & $n \cap 12$ & 0010 \\
\hline
\end{tabular}




\begin{tabular}{|c|c|c|c|c|c|c|c|}
\hline \multirow[b]{2}{*}{ Blocos } & \multicolumn{3}{|c|}{ REPETICAO VII } & \multirow[b]{2}{*}{ Blocos } & \multicolumn{2}{|c|}{ REPET $\mid C \bar{A} O$} & \multirow{2}{*}{$\frac{V 111}{3}$} \\
\hline & 1 & 2 & 3 & & 1 & 2 & \\
\hline & $200 n$ & $2 \cap 01$ & 2002 & & $20 \cap 0$ & 2001 & 2002 \\
\hline & 1200 & 1201 & 1202 & & 1200 & 1201 & 1202 \\
\hline & $0 \ln 0$ & 0101 & ก102 & & 0100 & 0101 & 0102 \\
\hline & 2120 & 2121 & 2122 & & 2120 & 2121 & 2122 \\
\hline & $102 \pi$ & 1021 & $1 \cap 22$ & & $102 n$ & 1021 & 1022 \\
\hline & $n 220$ & $n 221$ & $\cap 222$ & & $n 22 ?$ & 0221 & 0222 \\
\hline & 2210 & 2211 & 2212 & & 2210 & 2211 & 2212 \\
\hline & 1110 & 1111 & 1112 & & 1110 & 1111 & 1112 \\
\hline & nח & $n+11$ & $0 \cap 12$ & & 0010 & 0011 & $0 \cap 12$ \\
\hline & 2011 & 2012 & $2 \cap 10$ & & $2 n 12$ & 2010 & 2011 \\
\hline & 1211 & 1212 & 1210 & & 1212 & 1210 & 1211 \\
\hline & 0111 & 0112 & $n 110$ & & 0112 & 0110 & 0111 \\
\hline & 2101 & 2102 & 2100 & & 2102 & 2100 & 2101 \\
\hline & 1001 & $\ln \cap 2$ & $10 n n$ & & 1002 & 1000 & 1001 \\
\hline & 0201 & $02 \cap 2$ & $n 2 \cap n$ & & $\cap 2 \cap 2$ & 0200 & 2201 \\
\hline & 2.221 & 2222 & $222 ?$ & & 2222 & $222 n$ & 2221 \\
\hline & 1121 & 1122 & $112 n$ & & 1122 & $112 n$ & 1121 \\
\hline & $\cap \cap 21$ & $0 \cap 22$ & $n \cap 20$ & & $\ln 22$ & $\cap \cap 20$ & $0 \cap 21$ \\
\hline & 2022 & $2 \cap 2 ?$ & $2 \cap 21$ & & $2 \cap 21$ & $2 \cap 22$ & $2 \cap 2 \cap$ \\
\hline & 1222 & 1220 & 1221 & & 1221 & 1222 & 1220 \\
\hline & 0122 & $012 n$ & 0121 & & 0121 & 0122 & 0120 \\
\hline & 2112 & 2110 & 2111 & & 2111 & 2112 & 2110 \\
\hline & 1012 & $\ln 1 \cap$ & 1011 & & 1011 & $1 \cap 12$ & 1010 \\
\hline & 0212 & 0210 & $n 211$ & & $n 211$ & $n 212$ & $n 21 \pi$ \\
\hline & 2272 & $220 n$ & 2201 & & 2201 & $22 \cap 2$ & 2200 \\
\hline & 1102 & 1100 & 1101 & & 1101 & 1102 & 1100 \\
\hline & $\cap 002$ & $\cap \cap \cap \cap$ & $\cap \cap \cap 1$ & & $n \cap n 1$ & $\cap \cap 02$ & ח ח \\
\hline
\end{tabular}


b) RIncos de nove unidades

Interações confundidas:ADC, $A B D, A C D, B C D$ Informação relativa: $3 / 4$.

\begin{tabular}{|c|c|c|c|c|c|c|c|c|c|}
\hline \multirow[b]{2}{*}{ Blocos } & \multicolumn{9}{|c|}{ REPETICÃO 1} \\
\hline & 1 & 2 & 3 & 4 & 5 & 6 & 7 & 8 & 9 \\
\hline & $2 n n n$ & 2001 & $2 \cap n 2$ & $2 \ln n$ & 2101 & $2.1 \cap 2$ & $22 n n$ & 2201 & 2272 \\
\hline & $112 n$ & 1121 & 1122 & $122 n$ & 122.1 & 1222 & 1020 & 1021 & 1022 \\
\hline & 0210 & 0211 & $n 212$ & nnon & ח & $n 012$ & חו & חו & 0112 \\
\hline & 2111 & 2112 & 2110 & 22.11 & 2212 & $221 n$ & 2011 & 2012 & $2 n 10$ \\
\hline & $12 n 1$ & 1202 & 1200 & In & 1002 & 1000 & 1101 & 1102 & $110 n$ \\
\hline & 0021 & $\ln 22$ & $n \cap 2 n$ & 0121 & 0122 & 0120 & ก221 & 02.22 & 0220 \\
\hline & 2222 & 2220 & 22.1 & $2 \cap 22$ & 2020 & $2 \cap 21$ & 2122 & 2120 & 2121 \\
\hline & $\ln 12$ & 1010 & וחו & 1112 & 1110 & ו1ו & 1212 & $121 n$ & 1211 \\
\hline & $n \ln 2$ & ninn & 101 & $n 2 \cap 2$ & 0200 & $n 2 \cap 1$ & $\ln \cap 2$ & nnn & $n \cap n$ \\
\hline
\end{tabular}

\begin{tabular}{|c|c|c|c|c|c|c|c|c|c|}
\hline & \multicolumn{9}{|c|}{ REPETIC $\tilde{A}_{0} \quad 11$} \\
\hline Blocos & 1 & 2 & 3 & 4 & 5 & 6 & 7 & 8 & 9 \\
\hline & $2 n \cap n$ & 2001 & $20 \cap 2$ & 2100 & $2 \ln 1$ & $21 \cap 2$ & 2200 & 2201 & 2202 \\
\hline & 1117 & 1111 & 1112 & 1210 & 12.11 & 1212 & 1910 & 1011 & 1012 \\
\hline & $n 220$ & $n 221$ & 0222 & $\cap \cap 2 \cap$ & $n \cap 21$ & $0 \cap 2.2$ & 0120 & 0121 & ก 22 \\
\hline & 22.11 & 22.12 & 2210 & 2011 & 2012 & $2 \cap 1 \cap$ & 2111 & 2112 & 2111 \\
\hline & $\ln 21$ & $1 \cap 22$ & $\ln 2 n$ & 11121 & 1122 & $112 n$ & 1221 & 1222. & $122 ?$ \\
\hline & 0101 & 0102 & חחו & $n 2 \cap 1$ & $02 \cap 2$ & $02 n n$ & nnnl & ก)ก2 & nonn \\
\hline & 2122 & $212 n$ & 2121 & 2222 & 2220 & 2221 & $2 \cap 22$ & $2 \cap 2 n$ & 2021 \\
\hline & $12 \cap 2$ & 1200 & $12 n 1$ & $10 n 2$ & $10 n n$ & 1001 & $11 \cap 2$ & $110 n$ & 1101 \\
\hline & $n \cap 12$ & nוn & 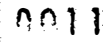 & 10112 & 0110 & ו1ו & $n 212$ & $n_{21}$ & 0211 \\
\hline
\end{tabular}




\begin{tabular}{|c|c|c|c|c|c|c|c|c|c|}
\hline & \multicolumn{9}{|c|}{ REPETIR.ÃO $\| 1$} \\
\hline Rlocos & 1 & 2 & 3 & 4 & 5 & 6 & 7 & 8 & 9 \\
\hline & 2000 & 2001 & 2002 & 2100 & 2101 & 2102 & 2200 & 2201 & 2202 \\
\hline & 1210 & 1211 & 1212 & 1010 & 1011 & 1012 & 1110 & 1111 & 1112 \\
\hline & 0120 & 0121 & 0122 & 02.20 & 0221 & 0222 & 0020 & 0021 & 0022 \\
\hline & 2.112 & 2.110 & 2111 & 2212 & 2210 & 22.11 & 2012 & 2010 & 2011 \\
\hline & 1022 & 1020 & 1021 & 1122 & 1120 & 1121 & 1222 & 1220 & 12.21 \\
\hline & 0202 & 0200 & 0201 & 0002 & 0000 & 0001 & 0102 & ח0 וח & 0101 \\
\hline & 2221 & 2222 & 2220 & 2021 & 2022 & 2020 & 2121 & 2122 & 2120 \\
\hline & 1101 & 1102 & 1100 & 1201 & $12 n 2$ & 1200 & 1001 & 1002 & 1000 \\
\hline & $0 \cap 11$ & 0012 & 0010 & 10111 & 22 & 0110 & 02.11 & $n 212$ & 0210 \\
\hline
\end{tabular}

\begin{tabular}{|c|c|c|c|c|c|c|c|c|c|}
\hline & \multicolumn{9}{|c|}{ REPETICAO } \\
\hline Blocos & 1 & 2 & 3 & 4 & 5 & 6 & 7 & 9 & 9 \\
\hline & $200 n$ & 2001 & 2002 & 2100 & 2101 & 2102 & $22 n n$ & 2201 & 2202 \\
\hline & 1220 & 1221 & 1222 & $\ln 20$ & 1021 & 1722 & 1120 & 112.1 & 1122 \\
\hline & 0 & חו & 0112 & 0210 & 0211 & 0212 & סוסחח & $n \cap 11$ & $n \cap 12$ \\
\hline & 2212 & 2210 & 2211 & $2 \cap 12$ & 2010 & 2011 & 2112 & 2110 & 2111 \\
\hline & $11 \cap 2$ & 1137 & 1101 & $12 \cap 2$ & 1200 & 1201 & 1002 & $\ln n n$ & 1001 \\
\hline & $\cap 022$ & $\cap \cap 2 n$ & $n \cap 21$ & 0122 & $n 12 n$ & 0121 & 0222 & 0220 & 10221 \\
\hline & 2121 & 2122 & $212^{n}$ & 2221 & 22.22 & $222 n$ & $2 \cap 21$ & 2022 & $2 \cap 20$ \\
\hline & 1011 & 1012 & $\ln 10$ & 1111 & 1112 & $111 n$ & 1211 & 1212 & 1210 \\
\hline & 0201 & $\cap 2 \cap 2$ & $n 2 \cap n$ & $\operatorname{nnn}$ & $\cap \cap \cap 2$ & nnn & וחוח & 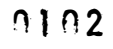 & $n 100$ \\
\hline
\end{tabular}

c) Blocos de três unidades

Interações confundidades: $A B, A C, A \cap, B C, B D, C D, A B C$, $A B \cap, A C D, B C \cap, A B C D$.

Informação relativa: $A B, A C, A D, B C, B D, C D: 1 / 2$ $A B C, A B D, A C D, B C D=3 / 4$ ABCO: $5 / 8$ 


\begin{tabular}{|c|c|c|c|c|c|c|c|c|c|}
\hline & \multicolumn{9}{|c|}{ REPETIÇÃO I } \\
\hline \multirow[t]{4}{*}{ Blocos } & 1 & 2 & 3 & 4 & 5 & 6 & 7 & 8 & 9 \\
\hline & 2000 & 2001 & 2002 & חוח & 2011 & 2012 & $2 \cap 20$ & 2021 & 2022 \\
\hline & 1111 & 1112 & 1110 & 1121 & 1122 & 1120 & 1101 & 1102 & 1100 \\
\hline & 0222 & $n 22 n$ & 0221 & $n 2 n 2$ & $n 20 n$ & 9201 & 0212 & $n 210$ & 0211 \\
\hline \multirow[t]{4}{*}{ Blocos } & 10 & 11 & 12 & 13 & 14 & 15 & 16 & 17 & 13 \\
\hline & 2100 & 2101 & $21 \cap 2$ & 2110 & 2111 & 2112 & $212 n$ & 2121 & 2122 \\
\hline & 1211 & 12.12 & $121 ?$ & 1221 & 1222 & $122 n$ & 1201 & 1202 & 1200 \\
\hline & $0 n 22$ & $n \cap 2 n$ & $n \cap 21$ & $\cap \cap \cap 2$ & nnn? & $0 \cap 31$ & 0012 & 0010 & $0 \cap 11$ \\
\hline \multirow[t]{4}{*}{ Blocos } & 19 & 20 & 21 & 22 & 23 & 24 & 25 & 26 & 27 \\
\hline & $22 n 0$ & $22 n 1$ & $22 \cap 2$ & $221 n$ & 2.211 & 2212 & $222 n$ & 2221 & 2222 \\
\hline & $\ln 11$ & 1012 & $\ln 1 n$ & $\ln 21$ & $1 \cap 22$ & $102 ?$ & 1001 & 1002 & 1030 \\
\hline & 0122 & $n 120$ & 0121 & 0172 & ninn & 0.101 & 10112 & $\ln 110$ & 0111 \\
\hline
\end{tabular}

\begin{tabular}{|c|c|c|c|c|c|c|c|c|c|}
\hline & \multicolumn{9}{|c|}{ REPETICAO 11} \\
\hline \multirow[t]{4}{*}{ Blocos } & 1 & 2 & 3 & 4 & 5 & 6 & 7 & 8 & 9 \\
\hline & $2 n n n$ & $2 \cap n 1$ & 2002 & 2010 & 2011 & $2 \cap 12$ & 2020 & 2021 & $2 \cap 22$ \\
\hline & 1112 & חווו & 1111 & 1122 & 1120 & 1121 & 1102 & $110 ?$ & 1101 \\
\hline & 0221 & 7222 & 02.20 & $n 201$ & $82 \cap 2$ & 7200 & 0211 & 0212 & 0211 \\
\hline \multirow[t]{4}{*}{ Blocos } & 10 & 11 & 12 & 13 & 14 & 15 & 16 & 17 & 18 \\
\hline & 2100 & 2101 & 2102 & 2110 & 2111 & 2112 & $212 n$ & 2121 & 2122 \\
\hline & 1212 & 1210 & 1211 & 12.22 & 1220 & 1221 & $12 \cap 2$ & $12 n 0$ & 1291 \\
\hline & 0.21 & $\cap \cap 22$ & $n \cap 2 n$ & ו & 0002 & 0000 & וחוח & 32 & 0010 \\
\hline \multirow[t]{4}{*}{ Blocos } & 19 & $2 n$ & 21 & 22 & 23 & 24 & 25 & 26 & 27 \\
\hline & 2200 & $22 n 1$ & 2202 & 2210 & 2211 & 2212 & 2220 & 2221 & 2222 \\
\hline & 1012 & ח1010 & 1011 & $1 \cap 22$ & 1020 & 1021 & 1002 & 1000 & $\ln 11$ \\
\hline & $n 121$ & 0122 & $012 n$ & וn & $\cap 1 \cap 2$ & ח & 0111 & 0112 & 3110 \\
\hline
\end{tabular}




\begin{tabular}{|c|c|c|c|c|c|c|c|c|c|}
\hline & \multicolumn{9}{|c|}{ REPETICAD 111} \\
\hline \multirow[t]{4}{*}{ Blocos } & 1 & 2 & 3 & 4 & 5 & 6 & 7 & 8 & 9 \\
\hline & 2000 & $2 n n 1$ & $20 \cap 2$ & $2 n 1 n$ & 2011 & 2012 & $202 n$ & 2021 & 2022 \\
\hline & 1121 & 1122 & 1120 & 1101 & 1102 & 1100 & 1111 & 1112 & 1110 \\
\hline & 0212 & $n 21 n$ & $n 211$ & $n 222$ & $022 n$ & 0221 & $\cap 2 \cap 2$ & $n 2 n n$ & 0201 \\
\hline \multirow[t]{4}{*}{ Blocos } & $\ln$ & 11 & 12 & 13 & 14 & 15 & 16 & 17 & 18 \\
\hline & $210 n$ & $21 \cap 1$ & $2 \ln 2$ & 2110 & 2111 & 2112 & 2120 & 2121 & 2122 \\
\hline & 1221 & 1222 & $122 n$ & $12 n 1$ & 1202 & 12.0 & 1211 & 1212 & 1210 \\
\hline & 0012 & 0010 & 3011 & $0 \cap 22$ & $082 n$ & $\operatorname{nn} 21$ & 0002 & 0000 & 0001 \\
\hline \multirow[t]{4}{*}{ Blocos } & 19 & $2 n$ & 21 & 22 & 23 & 24 & 25 & 26 & 2.7 \\
\hline & 2200 & 2201 & $22 \cap 2$ & 2210 & 2211 & 2212 & 2220 & 2221 & 2222 \\
\hline & 1021 & $1 \cap 22$ & 1020 & 1001 & 1002 & 1חnח & 1011 & 1012 & 1010 \\
\hline & IוI & ?ווח & 0111 & 0122 & 0120 & 0121 & 0102 & non & Iחוח \\
\hline
\end{tabular}

\begin{tabular}{|c|c|c|c|c|c|c|c|c|c|}
\hline & \multicolumn{9}{|c|}{ REPETICIOA } \\
\hline \multirow[t]{4}{*}{ Blocos } & 1 & 2 & 3 & 4 & 5 & 6 & 7 & 8 & 9 \\
\hline & 2030 & 2001 & $2 \cap \cap 2$ & 2010 & 2011 & 2012 & 2020 & 2021 & 2022 \\
\hline & 1122 & $112 n$ & 1121 & 1102 & 1100 & 1101 & 1112 & 1110 & 1111 \\
\hline & 0211 & 10212 & 0210 & 0221 & $n 222$ & 0220 & $020^{\circ}$ & 0202 & 0200 \\
\hline \multirow[t]{4}{*}{ Blocos } & in & 11 & 12 & 13 & 14 & 15 & 16 & 17 & 18 \\
\hline & 2100 & 2101 & $21 n 2$ & 2110 & 2111 & 2112 & 2120 & 2121 & 2122 \\
\hline & 1222 & 1220 & 1221 & $12 \cap 2$ & 1200 & 1201 & 1212 & 1210 & 1211 \\
\hline & 0011 & $n \cap 12$ & nnin & $\cap \cap 21$ & $n \cap 22$ & $n \cap 2 n$ & $n n n 1$ & $n 002$ & $0 n n n$ \\
\hline \multirow[t]{4}{*}{ Blocos } & 13 & 20 & 21 & 22 & 23 & 24 & 25 & 26 & 27 \\
\hline & 2200 & 2201 & $220 ?$ & 2210 & 2211 & 2212 & 2220 & 2221 & $2 ? 22$ \\
\hline & $\ln 22$ & 1020 & $1 \cap 21$ & $10 n 2$ & 1000 & 10101 & 1012 & $\ln 10$ & 1011 \\
\hline & וווח & 0112 & n & ח 12.1 & 0122 & $n 12 n$ & וחוח & 0102 & 0100 \\
\hline
\end{tabular}




\begin{tabular}{|c|c|c|c|c|c|c|c|c|c|}
\hline & \multicolumn{9}{|c|}{ REPETICAOO V } \\
\hline \multirow[t]{4}{*}{ Blocos } & 1 & 2 & 3 & 4 & 5 & 6 & 7 & 8 & 9 \\
\hline & 2000 & 2001 & 2002 & 2010 & 2011 & 2012 & $2 \cap 20$ & 2021 & $2 \cap 22$ \\
\hline & 1211 & 1212 & 1210 & 1221 & 1222 & $122 n$ & $12 n 1$ & 1202 & 1200 \\
\hline & 0122 & $n 120$ & $n 121$ & $n 1 \cap 2$ & 0100 & 0101 & 0112 & $n 110$ & 0111 \\
\hline \multirow[t]{4}{*}{ Blocos } & 10 & 11 & 12 & 13 & 14 & 15 & 16 & 17 & 18 \\
\hline & 2100 & 2191 & $21 \cap 2$ & 2110 & 2111 & 2112 & 2120 & 2121 & 2122 \\
\hline & 1011 & 1012 & n & $1 \cap 21$ & 1022 & 1020 & 1001 & $1 \cap \cap 2$ & $100 n$ \\
\hline & 0222 & 0220 & 0221 & $\cap 2 \cap 2$ & $n 2 n n$ & וס20 & $\cap 212$ & 1210 & 0211 \\
\hline \multirow[t]{4}{*}{ Blocos } & 19 & $2 ?$ & 21 & 22 & 23 & 24 & 25 & 26 & 27 \\
\hline & 2200 & $22 n 1$ & $22 \cap 2$ & 2.10 & 2211 & 2212 & $222 n$ & 22.21 & 2222 \\
\hline & 1111 & 1112 & 1110 & 1121 & 1122 & 1120 & 1101 & $11 \cap 2$ & 1100 \\
\hline & 0022 & $9 \cap 20$ & $0 \cap 21$ & $0 \cap 02$ & ח0חn & 1000 & Oחו2 & סוח0 & וI \\
\hline
\end{tabular}

\begin{tabular}{|c|c|c|c|c|c|c|c|c|c|}
\hline \multirow[b]{2}{*}{ Blocos } & \multicolumn{9}{|c|}{ REPET IC̃̃O } \\
\hline & 1 & 2 & 3 & 4 & 5 & 6 & 7 & 8 & 9 \\
\hline & 2000 & $20 n 1$ & 2002 & 2010 & 2011 & 2012 & 2020 & $2 \cap 21$ & 2022 \\
\hline & 1212 & 1210 & 12.11 & 1222 & 1220 & 12.21 & $12 n 2$ & 1200 & 1201 \\
\hline & 0121 & 0122 & $n 120$ & In & 0102 & 0100 & 0111 & 0112 & 0110 \\
\hline \multirow[t]{4}{*}{ Blocos } & 10 & 11 & 12 & 13 & 14 & 15 & 16 & 17 & 18 \\
\hline & 2100 & 2101 & 2102 & 2.110 & 2111 & 2112 & 2120 & 2121 & 2122 \\
\hline & 1012 & 1010 & 1011 & 1022 & 1020 & 1021 & 1002 & 1000 & 1001 \\
\hline & 0221 & 0222 & 0220 & 0201 & 0202 & 0200 & 0211 & 0212 & 0210 \\
\hline \multirow[t]{4}{*}{$01000 s$} & 19 & 20 & 21 & 22 & 23 & 24 & 25 & 26 & 27 \\
\hline & 2200 & 2201 & 2202 & 2210 & 2211 & 2212 & 2220 & 2221 & 2222 \\
\hline & 1112 & 1110 & 1111 & 1122 & 1120 & 1121 & 1102 & 1100 & 1101 \\
\hline & 0021 & 0022 & 0020 & 0001 & 0002 & 0000 & 0011 & 0012 & 0010 \\
\hline
\end{tabular}




\begin{tabular}{|c|c|c|c|c|c|c|c|c|c|}
\hline & \multicolumn{9}{|c|}{ REPETIEÃO VII } \\
\hline \multirow[t]{4}{*}{ Blocos } & 1 & 2 & 3 & 4 & 5 & 6 & 7 & 8 & 9 \\
\hline & 2000 & 2001 & 2002 & 2010 & 2011 & 2012 & 2020 & 2021 & $2 \cap 22$ \\
\hline & 1221 & 1222 & 1220 & 1201 & 1202 & $120 n$ & 1211 & 1212 & 1210 \\
\hline & 0112 & 0110 & n111 & 0122 & 0120 & 0121 & $n 1 \cap 2$ & nIno & 0101 \\
\hline \multirow[t]{4}{*}{ Dlocos } & 10 & 11 & 12 & 13 & 14 & 15 & 16 & 17 & 18 \\
\hline & 2100 & $2 \ln 1$ & $21 \cap 2$ & 2110 & 2.111 & 2112 & $212 n$ & 2121 & 2122 \\
\hline & 1021 & 1022 & $\ln 2 n$ & $10 n 1$ & 1000 & 1002 & 1011 & 1012 & 1010 \\
\hline & 0212 & 0210 & 0211 & 0222 & $022 n$ & 0221 & $\cap 2 \cap 2$ & $02 n n$ & $n 2 n 1$ \\
\hline \multirow[t]{4}{*}{ Blocos } & 19 & 20 & 21 & 22 & 23 & 24 & 25 & 26 & 27 \\
\hline & 2200 & $22 \cap 1$ & $22 \cap 2$ & $2.21 n$ & 2211 & 2212 & 2220 & 2221 & 2222 \\
\hline & 1121 & 1122 & 1120 & 1101 & $11 \cap 2$ & 1100 & 1111 & 11112 & 1110 \\
\hline & $n \cap 12$ & nnin & $n \cap 11$ & $n \cap 22$ & $n \cap 20$ & $0 \times 21$ & $\ln \cap 2$ & $n 073$ & 0001 \\
\hline
\end{tabular}

\begin{tabular}{|c|c|c|c|c|c|c|c|c|c|}
\hline & \multicolumn{9}{|c|}{ REPETICÃO } \\
\hline \multirow[t]{4}{*}{ กlocos } & 1 & 2 & 3 & 4 & 5 & 6 & 7 & 8 & 9 \\
\hline & 2000 & 2001 & 2002 & 2010 & 2011 & 2012 & 2020 & $2 \cap 21$ & 2022 \\
\hline & 1222 & $122 n$ & 1221 & 1202 & 1200 & 1201 & 1212 & 1210 & 1211 \\
\hline & 0111 & 0112 & 0110 & 0121 & ก122 & 0120 & 0101 & 0102 & 0100 \\
\hline \multirow[t]{4}{*}{ Blocos } & in & 11 & 12 & 13 & 14 & 15 & 16 & 17 & 18 \\
\hline & 2100 & 2101 & 2172 & 2110 & 2111 & 2112 & 2127 & 2121 & 2122 \\
\hline & $1 \cap 22$ & $\ln 20$ & 1021 & $10 n 2$ & $\ln n$ & $10 n 1$ & 1012 & 1010 & 1011 \\
\hline & 0211 & 0212 & 0210 & 0221 & $n 222$ & 0220 & 0201 & 0202 & 0200 \\
\hline \multirow[t]{4}{*}{ Dlocos } & 19 & 20 & 21 & 22 & 23 & 24 & 25 & 26 & 27 \\
\hline & 2200 & $22 . n 1$ & $22 \cap 2$ & 22.10 & 2211 & 2212 & 2220 & 222.1 & 2222 \\
\hline & 1122 & 1120 & 1121 & 1102 & 1100 & 1101 & 1112 & חו111 & 1111 \\
\hline & ווחס & 0012 & min & $\ln \cap 21$ & $0 \cap 22$ & $n \cap 20$ & $00 n 1$ & $0 \cap 72$ & 00090 \\
\hline
\end{tabular}


5.3. Fatoriais balanceados da série $3 \times 2^{n}$

5.3.1. Fatorial $3 \times 2$

Blocns de três unidades

Efeitos fatoriais confundidos: $B, A B$.

Informação relativa: $0: 3 / 9$

$$
A B: 5 / 9
$$

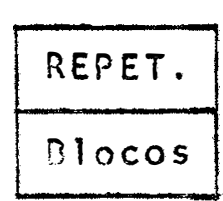

\begin{tabular}{|c|c|}
\hline \multicolumn{2}{|c|}{1} \\
\hline 1 & 2 \\
\hline $2 n$ & 21 \\
11 & $1 n$ \\
01 & 00 \\
\hline
\end{tabular}

\begin{tabular}{|c|c|}
\hline \multicolumn{2}{|c|}{11} \\
\hline 1 & 2 \\
\hline 10 & 11 \\
21 & 20 \\
01 & $0 n$ \\
\hline
\end{tabular}

\begin{tabular}{|c|c|}
\hline \multicolumn{2}{|c|}{111} \\
\hline 1 & 2 \\
\hline 00 & 01 \\
21 & $2 n$ \\
11 & 10 \\
\hline
\end{tabular}

5.3.2. Fatorial $3 \times 2^{2}$

a) Blocos de seis unidades

Interações confundidas: $B C, A B C$.

Informação relativa: BC: $8 / 9$

$A B C: 5 / 9$ 


\begin{tabular}{|c|c|c|c|c|c|c|}
\hline REPET. & \multicolumn{2}{|c|}{1} & \multicolumn{2}{|c|}{11} & \multicolumn{2}{|c|}{111} \\
\hline Blocos & 1 & 2 & 1 & 2 & 1 & 2 \\
\hline & $2 n n$ & 201 & $10 ?$ & 101 & non & 001 \\
\hline & 110 & 111 & $21 n$ & 211 & $21 ?$ & 211 \\
\hline & $n i n$ & 11 & nin & 011 & 110 & 111 \\
\hline & 211 & 210 & 111 & $11 n$ & 011 & nIn \\
\hline & 101 & $10 ?$ & 291 & $2 \cap n$ & 291 & 200 \\
\hline & $n \cap 1$ & $n 00$ & $0 \cap 1$ & $n \cap 0$ & 101 & $\ln n$ \\
\hline
\end{tabular}

b) Blocos de três unidades

Efeitos fatoriais confundidos: $B, C, B C, A B, A C, A B C$. Informação relativa: $R, C, B C: 8 / 9$

$A B, A C, A S C: 5 / 9$

\begin{tabular}{|c|c|c|c|c|}
\hline \multirow{2}{*}{$310 \cos$} & \multicolumn{4}{|c|}{ REPETICAO } \\
\hline & 1 & 2 & 3 & 4 \\
\hline & 200 & 201 & 210 & 211 \\
110 & 111 & 100 & 101 \\
& 011 & 010 & 001 & 000 \\
\hline
\end{tabular}

\begin{tabular}{|c|c|c|c|c|}
\hline & \multicolumn{4}{|c|}{ REPETICÃO II } \\
\hline Blocos & 1 & 2 & 3 & 4 \\
\hline & 100 & 101 & 110 & 111 \\
\hline & 211 & 210 & 201 & 200 \\
\hline & nin & II & $n \cap 0$ & nOl \\
\hline
\end{tabular}




\begin{tabular}{|c|c|c|c|c|}
\hline \multirow{2}{*}{} & \multicolumn{4}{|c|}{ REPETIOXO 11} \\
\hline BIocos & 1 & 2 & 3 & 4 \\
\hline & 000 & 001 & 010 & 011 \\
210 & 211 & 200 & 201 \\
111 & 110 & 101 & 100 \\
\hline
\end{tabular}

5.3.3. Fatorial $3 \times 2^{3}$

a) Blocos de doze unidades

Interações confundidas: CCD, ADCD.

Informação relativa: ncก: $8 / 9$

ABCD: $5 / 9$

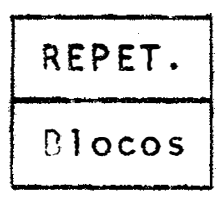

\begin{tabular}{|c|c|}
\hline \multicolumn{2}{|c|}{1} \\
\hline 1 & 2 \\
\hline 2000 & 2001 \\
1100 & 1101 \\
$010 n$ & 0101 \\
2110 & 2111 \\
1010 & 1011 \\
0010 & $n 011$ \\
2011 & 2010 \\
1111 & 1110 \\
0111 & 0110 \\
2101 & 2100 \\
1001 & $10 n 0$ \\
$0 n 01$ & $0 n 00$ \\
\hline
\end{tabular}

\begin{tabular}{|c|c|}
\hline \multicolumn{2}{|c|}{11} \\
\hline 1 & 2 \\
\hline 1000 & 1001 \\
2100 & 2101 \\
0100 & 0101 \\
1110 & 1111 \\
2010 & 2011 \\
$0 n 10$ & 0011 \\
$1 n 11$ & 1010 \\
2111 & 2110 \\
0111 & 0110 \\
1101 & 1100 \\
$20 n 1$ & 2000 \\
$0 n n 1$ & 0000 \\
\hline
\end{tabular}

\begin{tabular}{|c|c|}
\hline \multicolumn{2}{|c|}{111} \\
\hline 1 & 2 \\
\hline$n 000$ & 0001 \\
$210 n$ & 2101 \\
1100 & 1101 \\
$n 11 n$ & 0111 \\
2010 & 2011 \\
1010 & 1011 \\
0011 & 0010 \\
2111 & 2110 \\
1111 & 1110 \\
$n 101$ & $010 n$ \\
2001 & $20 n n$ \\
$10 n 1$ & $100 n$ \\
\hline
\end{tabular}


b) Blocos te seis unidades

Interações confundidas: $B C, B D, C D, A B C$, $A B D, A C D$.

Informação relativa: $B C, B D, C D: 8 / 9$ $A B C, A B D, A C D: 5 / 9$

\begin{tabular}{|c|c|c|c|c|}
\hline \multicolumn{4}{c|}{} & \multicolumn{4}{|c|}{ REPETICÄO } \\
\hline Blocos & 1 & 2 & 3 & 4 \\
\hline & 2000 & 2001 & 2010 & 2011 \\
1100 & 1101 & 1110 & 1111 \\
0110 & 0111 & 0100 & 0101 \\
2111 & 2110 & 2101 & 2100 \\
1011 & 1010 & 1001 & 1000 \\
$0 n 01$ & $n 000$ & 0011 & 0010 \\
\hline
\end{tabular}

\begin{tabular}{|c|c|c|c|c|}
\hline \multicolumn{4}{c|}{ REPETICÃO 11 } \\
\hline \multirow{1}{*}{ Blocos } & 1 & 2 & 3 & 4 \\
\hline 1000 & 1001 & 1010 & 1011 \\
2110 & 2111 & 2100 & 2101 \\
0100 & 0101 & 0110 & 0111 \\
1111 & 1110 & 1101 & 1100 \\
2001 & 2000 & 2011 & 2010 \\
$0 n 11$ & 0010 & 0001 & 0000 \\
\hline
\end{tabular}




\begin{tabular}{|c|c|c|c|c|}
\hline \multicolumn{4}{c|}{ REPETICAOO } & \multicolumn{1}{|c|}{11} \\
\hline Blocos & 1 & 2 & 3 & 4 \\
\hline 0000 & 0001 & 0010 & 0011 \\
2100 & 2101 & 2110 & 2111 \\
1110 & 1111 & 1100 & 1101 \\
0111 & 0110 & 0101 & 0100 \\
2011 & 2010 & 2001 & 2000 \\
1001 & 1000 & 1011 & 1010 \\
\hline
\end{tabular}

5.4. Fatoriats balanceados da série $3^{2} \times 2^{n}$

5.4.1. Fatorial $3^{2} \times 2$

a) Blocos de nove unidades

Efeitos fatoriais confundidos: $B, B C$.

Informação relativa: $C: 8 / 9, B C: 5 / 9$.

\begin{tabular}{|c|c|c|c|c|c|c|}
\hline REPET. & \multicolumn{2}{|c|}{1} & \multicolumn{2}{|c|}{11} & \multicolumn{2}{|c|}{111} \\
\hline Blocos & 1 & 2. & 1 & 2. & 1 & 2. \\
\hline & 020 & 021 & 010 & 011 & 000 & 001 \\
\hline & 011 & 0 & 021 & 020 & 021 & 020 \\
\hline & 001 & 000 & 001 & 000 & 011 & 010 \\
\hline & 120 & 121 & 117 & 111 & 100 & 101 \\
\hline & 111 & 110 & 121 & $12 n$ & 121 & 120 \\
\hline & 101 & $10 n$ & 101 & 100 & 111 & 110 \\
\hline & $22 n$ & 221 & 210 & 211 & 200 & 201 \\
\hline & 211 & 210 & 221 & $22 n$ & 221 & 227 \\
\hline & $2 \cap 1$ & 200 & 201 & 200 & 211 & $21 n$ \\
\hline
\end{tabular}


b) Blocos de seis unidades

Interações confundidas: AR, AnC.

Informação relativa: $A B: 7 /\{$

Anr: $5 / 8$
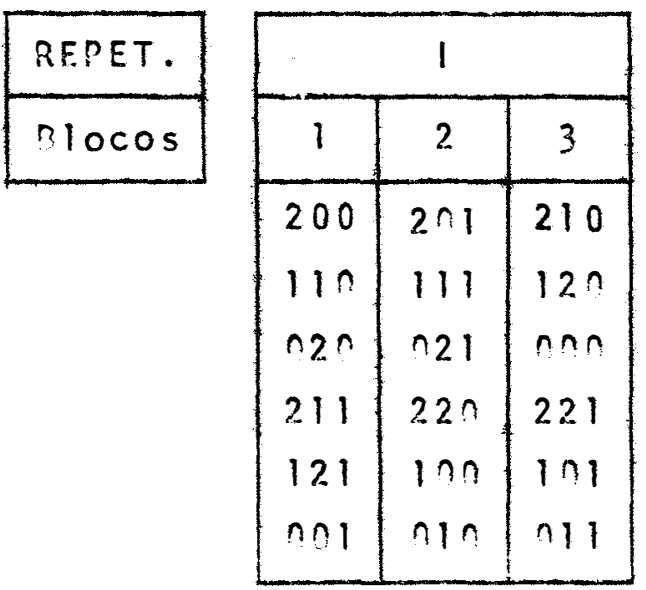

\begin{tabular}{|c|c|c|}
\hline \multicolumn{3}{|c|}{11} \\
\hline 1 & 2 & 3 \\
\hline $2 n 1$ & $20 n$ & 211 \\
111 & $11 n$ & 121 \\
$n 21$ & $n 20$ & $n n 1$ \\
210 & 221 & $22 n$ \\
$12 n$ & $1 n 1$ & $1 n n$ \\
$n n n$ & $n 11$ & $n 10$ \\
\hline
\end{tabular}

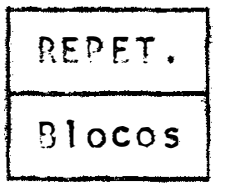

\begin{tabular}{|c|c|c|}
\hline \multicolumn{3}{|c|}{111} \\
\hline 1 & 2 & 3 \\
\hline 200 & 201 & 210 \\
120 & 121 & $10 n$ \\
$n 1 n$ & $n 11$ & $n 20$ \\
211 & $22 n$ & 221 \\
101 & 110 & 111 \\
$n 21$ & $n n n$ & $n n 1$ \\
\hline
\end{tabular}

\begin{tabular}{|c|c|c|}
\hline \multicolumn{3}{|c|}{11} \\
\hline 1 & 2 & 3 \\
\hline $2 n 1$ & $2 n n$ & 211 \\
121 & $12 n$ & $1 n 1$ \\
$n 11$ & $n 1 n$ & $n 21$ \\
$21 n$ & 221 & 220 \\
$1 n n$ & 111 & $11 n$ \\
$n 2 n$ & $n n 1$ & $n n n$ \\
\hline
\end{tabular}

c) Blocos de três unidades

Efeitos fatoriais confundidos: $C, A B, A C, B C, A B C$. Informação relativa: $C: \cap / 9 ; A B: 1 / 2 ; A C, O C: 5 / 9$ An: : $13 / 18$. 


\begin{tabular}{|c|c|c|c|c|c|c|}
\hline & \multicolumn{6}{|c|}{ REPFTICAn } \\
\hline Blocos & 1 & 2 & 3 & 4 & 5 & 6 \\
\hline & $n 2 n$ & $12 n$ & $22 n$ & 921 & 121 & 221 \\
& 111 & 211 & $n 11$ & 110 & $21 n$ & $n 10$ \\
& $2 n 1$ & $n n 1$ & $1 n 1$ & $2 n n$ & $n n n$ & $10 n$ \\
\hline
\end{tabular}

\begin{tabular}{|c|c|c|c|c|c|c|}
\hline & \multicolumn{6}{|c|}{ PEPETICÃO 11} \\
\hline Dlocos & 1 & 2. & 3 & 4 & 5 & 6 \\
\hline & $n 2 n$ & $12 n$ & $22 n$ & 721 & 12.1 & 221 \\
\hline & 211 & 011 & 111 & 210 & 010 & 110 \\
\hline & $\ln 1$ & $2 \cap 1$ & $n \cap 1$ & $\ln n$ & $20 n$ & $n \cap n$ \\
\hline
\end{tabular}

\begin{tabular}{|c|c|c|c|c|c|c|}
\hline & \multicolumn{6}{|c|}{ REOETICÃO } \\
\hline locos & 1 & 2 & 3 & 4 & 5 & 6 \\
\hline & $n ! n$ & 110 & $21 n$ & $n 11$ & 111 & 211 \\
\hline & 121 & 22.1 & 721 & 120 & $22 n$ & $\because 2 \cap$ \\
\hline & $2 n 1$ & $n \cap 1$ & 101 & 200 & non & $10 n$ \\
\hline
\end{tabular}

\begin{tabular}{|c|c|c|c|c|c|c|}
\hline & \multicolumn{6}{|c|}{ REPETICÃก IV } \\
\hline Dlocos & 1 & 2 & 3 & 4 & 5 & 6 \\
\hline & nin & 110 & $21 n$ & 011 & 111 & 211 \\
\hline & 2.21 & 021 & 12.1 & 220 & $n 2 ?$ & $12 n$ \\
\hline & 101 & 201 & $n \cap 1$ & $10 n$ & $20 n$ & nno \\
\hline
\end{tabular}




\begin{tabular}{|c|c|c|c|c|c|c|}
\hline & \multicolumn{6}{|c|}{ REPET I ĢAO } \\
\hline Blocos & 1 & 2 & 3 & 4 & 5 & 6 \\
\hline & חח & inn & $2 n n$ & וחח & וחו & $2 \cap 1$ \\
\hline & 121 & 221 & $\cap 21$ & $12 n$ & 220 & $02 n$ \\
\hline & 211 & $n \mid l$ & 111 & 210 & nin & ח11 \\
\hline
\end{tabular}

\begin{tabular}{|c|c|c|c|c|c|c|}
\hline & \multicolumn{6}{|c|}{ REPETIRAO VI } \\
\hline Blocos & 1 & 2 & 3 & 4 & 5 & 6 \\
\hline & $n \cap n$ & 100 & $2 \cap 0$ & $n \cap 1$ & 101 & 201 \\
\hline & 221 & $n 21$ & 12.1 & $22 \pi$ & $72 n$ & $12 n$ \\
\hline & 111 & 2.11 & וा & 110 & $21 n$ & 010 \\
\hline
\end{tabular}

5.4.2. Fatortal $3^{2} \times 2^{2}$

a) Blncos de dezoito unidades

Interações confundidas: CD, R,CD

Informação relativa: c c : $8 / 9$

$\operatorname{BCD}: 5 / 9$ 


\begin{tabular}{|c|c|c|c|c|c|c|}
\hline REPET. & \multicolumn{2}{|c|}{1} & \multicolumn{2}{|c|}{11} & \multicolumn{2}{|c|}{111} \\
\hline Elocos & 1 & 2 & 1 & 2 & 1 & 2 \\
\hline & 0200 & $n 2 \cap 1$ & $n \ln$ & $n 101$ & non & nกก \\
\hline & 6116 & 0111 & 0210 & 0211 & 0210 & 0211 \\
\hline & noin & Iח & nnin & $n \cap 11$ & 0110 & 0111 \\
\hline & $120 ?$ & $12 n 1$ & 1100 & 110 & $\ln n$ & $\operatorname{lnn} 1$ \\
\hline & 1110 & 1111 & $121 n$ & 1211 & 1210 & 1211 \\
\hline & 1910 & $1 \cap 11$ & 1010 & $1 \cap 11$ & 1110 & 1111 \\
\hline & $22 \cap 0$ & $22 n 1$ & 2100 & 2191 & $2 \cap \cap n$ & $2 \cap 01$ \\
\hline & 2110 & 2111 & 2.210 & 22.11 & 2210 & 2211 \\
\hline & $2 \cap 1 n$ & 2011 & 2710 & 2011 & $211 n$ & 2111 \\
\hline & ก211 & 210 & $n 111$ & $n 110$ & nกוा & $n n i n$ \\
\hline & In & ninn & $n 2 n 1$ & $n 2 \cap n$ & $n 2 \cap 1$ & $n 2 n n$ \\
\hline & וnח & חกח & $n \cap n$ & $n \cap \cap n$ & ו & $n 10 n$ \\
\hline & 1211 & 1217 & 1111 & 1117 & 1011 & $\ln 10$ \\
\hline & 1101 & $110 n$ & $12 n 1$ & 1207 & 1201 & $12 \cap ?$ \\
\hline & $1 \cap n 1$ & $\ln n$ & $1 \cap n 1$ & $\ln n$ & $11 \cap 1$ & $110 n$ \\
\hline & 2211 & 2213 & 2111 & $?$ & 2011 & $2 n 10$ \\
\hline & $21 \div 1$ & $21 \cap n$ & $22 \cap 1$ & $220 n$ & $22 n 1$ & $22 n n$ \\
\hline & $20 \cap 1$ & $2 n \cap n$ & $2 \cap n 1$ & $2 n \cap n$ & 2101 & $21 \cap n$ \\
\hline
\end{tabular}

b) Blocos de doze unidades

Interações confundidas: $A$ ? $A \cap C, A \cap n, A B C$.

Informação relativa: $A ?: 31 / 32$

ARC, ABD: 29/32

AกCD: $23 / 32$ 


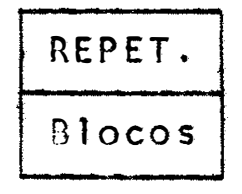

\begin{tabular}{|c|c|c|}
\hline \multicolumn{3}{|c|}{1} \\
\hline 1 & 2 & 3 \\
\hline $200 n$ & $2 n n 1$ & $201 n$ \\
$110 n$ & $11 n 1$ & 1110 \\
$n 20 n$ & $n 201$ & 0210 \\
$211 n$ & 2111 & $22 n n$ \\
$121 n$ & 1211 & $10 n n$ \\
$n n 10$ & $n n 11$ & $n 1 n n$ \\
$2 n 11$ & $21 n n$ & $21 n 1$ \\
1111 & $120 n$ & $12 n 1$ \\
$n 211$ & $n n n n$ & $n n n 1$ \\
$22 n 1$ & $221 n$ & 2211 \\
$1 n n 1$ & 1010 & $1 n 11$ \\
$n 131$ & $n 11 n$ & $n 111$ \\
\hline
\end{tabular}

\begin{tabular}{|c|c|c|}
\hline \multicolumn{3}{|c|}{11} \\
\hline 1 & 2 & 3 \\
\hline 2001 & $2 \cap \cap \cap$ & 2011 \\
\hline 1111 & $11 \cap ?$ & 1111 \\
\hline$n 2 \cap 1$ & $\cap 2 \cap 0$ & 0211 \\
\hline 2111 & 2110 & $22 \cap 1$ \\
\hline 1211 & 1210 & $1 \cap n 1$ \\
\hline ח & nกIก & וn \\
\hline $2 \cap 1 \cap$ & $21 \cap 1$ & $21 \cap 0$ \\
\hline 1110 & $12 \cap 1$ & $12 \cap 0$ \\
\hline$n 210$ & $n \cap \cap 1$ & nnกn \\
\hline $22 ? !$ & 2211 & 2210 \\
\hline $\operatorname{lnn} n$ & $1 \cap 11$ & 1010 \\
\hline$n \operatorname{nn}$ & 0111 & 0110 \\
\hline
\end{tabular}

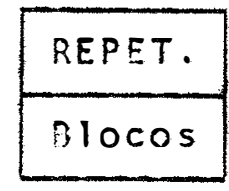

\begin{tabular}{|c|c|c|}
\hline \multicolumn{3}{|c|}{111} \\
\hline 1 & 2 & 3 \\
\hline 2010 & $2 n 11$ & $2 n n n$ \\
$111 n$ & 1111 & $11 n n$ \\
$n 21 n$ & 0211 & $n 2 n n$ \\
$21 n n$ & $21 n 1$ & $221 n$ \\
$12 n n$ & $12 n 1$ & $1 n 1 n$ \\
$n n n n$ & $n n n 1$ & $n 11 n$ \\
$2 n n 1$ & $211 n$ & 2111 \\
$11 n 1$ & 1210 & 1211 \\
$n 2 n 1$ & $n n 1 n$ & $n n 11$ \\
2211 & $22 n n$ & $22 n 1$ \\
$1 n 11$ & $1 n n n$ & $10 n 1$ \\
$n 111$ & $n 1 n n$ & $n 1 n 1$ \\
\hline
\end{tabular}

\begin{tabular}{|c|c|c|}
\hline \multicolumn{3}{|c|}{ IV } \\
\hline 1 & 2 & 3 \\
\hline 2011 & 2010 & $2 \cap n 1$ \\
\hline 1111 & 1110 & 1101 \\
\hline ก211 & $\cap 21 \cap$ & 7201 \\
\hline 2101 & $21 \cap 0$ & 2211 \\
\hline 1201 & 1200 & 1711 \\
\hline$\cap \cap \cap 1$ & กחก? & 0111 \\
\hline $2 \cap \cap \cap$ & 2111 & 2110 \\
\hline 1100 & 12.11 & 1210 \\
\hline $72 \cap n$ & 0011 & nnln \\
\hline $221 n$ & $22 \cap 1$ & $22 \cap 0$ \\
\hline 1910 & 1031 & $10 n$ \\
\hline$? 110$ & 0101 & nIn \\
\hline
\end{tabular}


132.

\begin{tabular}{|c|c|c|c|c|c|c|}
\hline REPET. & \multicolumn{3}{|c|}{$y$} & \multicolumn{3}{|c|}{$|V|$} \\
\hline Blocos & 1 & 2 & 3 & 1 & 2 & 3 \\
\hline & $2 n \cap n$ & $2 \cap \div 1$ & $201 ?$ & $2 \cap n 1$ & 2000 & 2011 \\
\hline & $12 n n$ & $12 n 1$ & 1213 & $12 n 1$ & $120 n$ & 1211 \\
\hline & nin & $n 101$ & 0110 & 0101 & $010 n$ & 0111 \\
\hline & 2119 & 2111 & $22 \cap n$ & 2111 & $211 n$ & 2201 \\
\hline & 1010 & 1011 & $11 n n$ & $1 \cap 11$ & 1010 & $11 \cap 1$ \\
\hline & $n 210$ & 0211 & nกก & 0211 & $n 21 n$ & nก: \\
\hline & 2011 & 2100 & $21 n$ & $2 \cap 1 n$ & $21 \cap 1$ & $210 n$ \\
\hline & 1211 & Iกกก & 1001 & 1210 & $\operatorname{lnn} 1$ & 1000 \\
\hline & 0111 & $n 2 n n$ & $02 \cap 1$ & 9110 & 0201 & ก?กn \\
\hline & 2271 & 2210 & 2211 & $22 n n$ & 2211 & 2.10 \\
\hline & 1101 & 1117 & 1111 & $110 n$ & 1111 & 1110 \\
\hline & $n \cap \cap 1$ & $n \cap i n$ & $0 \cap 11$ & חกח & $0 \cap 11$ & ก) \\
\hline
\end{tabular}

\begin{tabular}{|c|c|c|c|c|c|c|}
\hline REPET. & \multicolumn{3}{|c|}{ VII } & \multicolumn{3}{|c|}{ VIII } \\
\hline Blocos & 1 & 2 & 3 & 1 & 2 & 3 \\
\hline & 2010 & 2011 & $20 \cap 0$ & 2011 & 2010 & 2001 \\
\hline & 1210 & 1211 & 12.00 & 1211 & 1210 & $12 n 1$ \\
\hline & 0110 & וווח & ninn & וו & $n 110$ & In \\
\hline & 2100 & $2 \ln 1$ & 2210 & 2131 & $210 n$ & 22.11 \\
\hline & IOAO & 1001 & 1110 & וחו & $\ln n ?$ & 1111 \\
\hline & $n 2^{n n}$ & $n 2 \cap 1$ & noln & $n 2 \cap 1$ & $n 2 \cap n$ & וותח \\
\hline & $20 n 1$ & 2110 & 2111 & $2 \cap n n$ & 2.111 & 2110 \\
\hline & $12 n 1$ & $\ln 10$ & 1011 & $120 n$ & $\ln 11$ & $\ln 10$ \\
\hline & nin & $n 21 n$ & ก211 & $910 ?$ & 0211 & $n 210$ \\
\hline & 2211 & $22 n n$ & $22 n 1$ & $221 n$ & $22 n 1$ & 2200 \\
\hline & 1111 & $110 n$ & 1101 & 1110 & 1101 & $\operatorname{linn}$ \\
\hline & ו & nnno & nחnl & $n$ & $n \cap n ו$ & $n \cap \cap n$ \\
\hline
\end{tabular}


c) Blocos de seis unidades

Interações confundidas: AR, CD, ACD, ABCD.

Informação relativa: Af: $1 / 2$

$$
\text { CD: } 8 / 9
$$

ACก: $5 / 3$

ACCO: $13 / 18$

\begin{tabular}{|c|c|c|c|c|c|c|}
\hline & \multicolumn{6}{|c|}{ REPETICÃO I } \\
\hline $31000 \mathrm{~s}$ & 1 & 2 & 3 & 4 & 5 & 6 \\
\hline & $020 n$ & 0201 & 1200 & 1201 & 2200 & 2201 \\
\hline & 1110 & 1111 & 2119 & 2111 & ח110 & 0111 \\
\hline & 2010 & 2011 & 0010 & $0 \cap 11$ & 1010 & 1011 \\
\hline & 0211 & 0210 & 1211 & 12.10 & 3211 & 2210 \\
\hline & $11 \cap 1$ & 1100 & 2101 & 2100 & 0101 & 0100 \\
\hline & $2 \cap n 1$ & 2000 & 0001 & nחกn & 1001 & 1000 \\
\hline
\end{tabular}

\begin{tabular}{|c|c|c|c|c|c|c|}
\hline \multirow[b]{2}{*}{ Blocos } & \multicolumn{6}{|c|}{ REPETIÇÃO } \\
\hline & 1 & 2 & 3 & 4 & 5 & 6 \\
\hline & 0200 & 0201 & 1200 & 1201 & 2200 & 2201 \\
\hline & ח11 & 2111 & n & 0111 & 1110 & 1111 \\
\hline & חוחו & 1011 & 2010 & 2011 & 0010 & 0011 \\
\hline & 0211 & 0210 & 1211 & 1210 & 2211 & 22.10 \\
\hline & 2101 & 2100 & וחוח & 0100 & 1101 & $110 n$ \\
\hline & 1001 & 1000 & $20 n 1$ & 2000 & ו & 0000 \\
\hline
\end{tabular}




\begin{tabular}{|c|c|c|c|c|c|c|}
\hline & \multicolumn{6}{|c|}{ REPETICAO $\| 11$} \\
\hline Blocos & 1 & 2 & 3 & 4 & 5 & 6 \\
\hline & ninn & 0101 & $110 n$ & 1101 & 2.100 & 2101 \\
\hline & 1210 & 1211 & 2210 & 2211 & 0210 & 9211 \\
\hline & 2010 & 2011 & 0010 & 0011 & 1010 & $\ln 11$ \\
\hline & 0111 & חו & 1111 & 1110 & 2111 & 2110 \\
\hline & 1201 & 1200 & 2201 & 2200 & $02 \cap 1$ & 0200 \\
\hline & 2001 & $20 n n$ & 001 & $n 000$ & 1001 & $10 n 0$ \\
\hline
\end{tabular}

\begin{tabular}{|c|c|c|c|c|c|c|}
\cline { 2 - 7 } & \multicolumn{7}{|c|}{ REPETICAO IV } \\
\hline Blocos & 1 & 2 & 3 & 4 & 5 & 6 \\
\hline & 0100 & 0101 & 1100 & 1101 & 2100 & 2101 \\
2210 & 2211 & 0210 & 3211 & 1210 & 1211 \\
1010 & 1011 & 2010 & 2011 & 0010 & 0011 \\
& 0111 & 0110 & 1111 & 1110 & 2111 & 2110 \\
& 2201 & 2200 & 201 & 0200 & 1201 & 1230 \\
1001 & 1000 & 2001 & 2000 & 0001 & 0300 \\
\hline
\end{tabular}

\begin{tabular}{|c|c|c|c|c|c|c|}
\hline \multirow[b]{2}{*}{$31000 \mathrm{~s}$} & \multicolumn{6}{|c|}{ REPETIC苂O V } \\
\hline & 1 & 2. & 3 & 4 & 5 & 6 \\
\hline & $000 n$ & nחnl & 1000 & 1001 & $200 n$ & $2 \cap 01$ \\
\hline & 12.10 & 1211 & 2.10 & 2211 & $n 210$ & 7211 \\
\hline & 2110 & 2111 & חו & ח11 & n111 & 111 \\
\hline & ח & 3017 & 1011 & 1010 & 2011 & 2010 \\
\hline & $12 \cap 1$ & 1200 & 2201 & 2200 & 7201 & 0200 \\
\hline & 2101 & 2100 & 010 & nino & 1101 & 1100 \\
\hline
\end{tabular}




\begin{tabular}{|c|c|c|c|c|c|c|}
\hline \multicolumn{1}{|c|}{} & \multicolumn{6}{|c|}{ REPETICAO VI } \\
\hline Blocos & 1 & 2 & 3 & 4 & 5 & 6 \\
\hline & 0000 & 0001 & 1000 & 1001 & 2000 & 2001 \\
2210 & 2211 & 0210 & 0211 & 1210 & 1211 \\
1110 & 1111 & 2110 & 2111 & 0110 & 0111 \\
$0 n 11$ & 0010 & 1011 & 1010 & 2011 & 2010 \\
2201 & 2200 & 0201 & 0200 & 1201 & 1200 \\
1101 & 1100 & 2101 & 2100 & 0101 & 0100 \\
\hline
\end{tabular}

5.5. Fatoriais balanceados da série $3^{3} \times 2^{n}$

5.5.1. Fatorial $3^{3} \times 2$

a) Blocos de doze unidades

Interações confundidas: $A B C, A B C D$

Informação relativa: ABC: $15 / 16$

$A B C D: 13 / 16$ 


\begin{tabular}{|c|c|c|c|c|c|c|}
\hline REPET. & \multicolumn{3}{|c|}{1} & \multicolumn{3}{|c|}{11} \\
\hline Blocos & 1 & 2 & 3 & 1 & 2 & 3 \\
\hline & 2000 & 2001 & 2010 & $2 \cap \cap 1$ & $200 n$ & 2011 \\
\hline & $110 ?$ & 1101 & 1110 & $11 \cap 1$ & 1100 & 1111 \\
\hline & 0200 & 0201 & 0210 & $\cap 2 \cap 1$ & $020 n$ & 0211 \\
\hline & 2110 & 2111 & 2120 & 2111 & 2110 & 2121 \\
\hline & 1210 & 1211 & 1220 & 1211 & 1210 & 1221 \\
\hline & 0010 & 0011 & $072 n$ & 0011 & 0010 & $\ln 21$ \\
\hline & 2220 & 2221 & 2200 & 2231 & $222 \pi$ & $22 \div 1$ \\
\hline & 1020 & 1021 & 1000 & 11121 & 1020 & 1001 \\
\hline & 0120 & 0121 & 0100 & 0121 & $012 ?$ & 2101 \\
\hline & 2011 & 2020 & 2021 & 2017 & 2021 & $2 \div 20$ \\
\hline & 1111 & 1120 & 1121 & 1110 & 1121 & 1120 \\
\hline & 0211 & 0220 & 0221 & 0210 & 0221 & 0220 \\
\hline & 2121 & $2 \ln$ & $21 \cap 1$ & 2120 & 2101 & 2170 \\
\hline & 1221 & $12 n n$ & 1201 & $122 ?$ & $12 n 1$ & 1200 \\
\hline & $0 \cap 21$ & 0000 & nกn & 0020 & $0 \cap 01$ & 0000 \\
\hline & 2201 & 2210 & 2211 & $220 ?$ & 2211 & 2210 \\
\hline & 1001 & 1010 & 1011 & $100 n$ & 1011 & 1010 \\
\hline & 0101 & 0110 & 0111 & 0100 & 0111 & 0110 \\
\hline
\end{tabular}




\begin{tabular}{|c|c|c|c|c|c|c|}
\hline REPET. & \multicolumn{3}{|c|}{111} & \multicolumn{3}{|c|}{ IV } \\
\hline \multirow[t]{19}{*}{ Blocos } & 1 & 2 & 3 & 1 & 2 & 3 \\
\hline & 2000 & 2001 & 2010 & $20 n 1$ & $200 n$ & 2011 \\
\hline & 1100 & 1101 & 1110 & 1101 & $110 ?$ & 1111 \\
\hline & 0200 & 0201 & ก21ก & 0201 & $02 \cap 0$ & 0211 \\
\hline & 2120 & 2121 & $210 ?$ & 2121 & 2120 & 2101 \\
\hline & 1220 & 1221 & 1200 & 1221 & $122 ?$ & 1201 \\
\hline & $002 n$ & $n \cap 21$ & 3000 & 0021 & $002 \cap$ & 0001 \\
\hline & 2210 & 2211 & $222 n$ & 2211 & $221 \pi$ & 2221 \\
\hline & 1010 & 1011 & 1020 & 1011 & 1010 & 1021 \\
\hline & 0110 & 0111 & 0120 & 0111 & $011 n$ & 0121 \\
\hline & 2011 & 2020 & 2021 & 2010 & 2021 & $2 \cap 20$ \\
\hline & 1111 & 1120 & 1121 & 1110 & 1121 & 1120 \\
\hline & 0211 & 0220 & 0321 & $021 ?$ & 0221 & 0220 \\
\hline & 2101 & 2110 & 2111 & 2100 & 2111 & 2.110 \\
\hline & 1201 & 1210 & 1211 & 1200 & 1211 & 1210 \\
\hline & 0001 & $n \cap 10$ & กกा & 0000 & $n \cap 11$ & 0010 \\
\hline & 2221 & $22 \cap 0$ & 2201 & 2220 & 2201 & $22 \cap 0$ \\
\hline & 1021 & $\ln n$ & 1091 & 1020 & 1001 & $100 n$ \\
\hline & 0121 & 000 & 0101 & 0120 & 0101 & 0100 \\
\hline
\end{tabular}




\begin{tabular}{|c|c|c|c|c|c|c|}
\hline REPET. & \multicolumn{3}{|c|}{ V } & \multicolumn{3}{|c|}{ VI } \\
\hline \multirow[t]{19}{*}{ Blocos } & 1 & 2 & 3 & 1 & 2 & 3 \\
\hline & 2000 & 2001 & $2 n 10$ & 2001 & $2 \cap \cap n$ & 2011 \\
\hline & 1200 & 1201 & 1210 & 1201 & 1200 & 1211 \\
\hline & 0100 & 0101 & ח11 & 019 & $n \ln$ & 0111 \\
\hline & 2110 & 2.111 & $212^{n}$ & 2111 & 2110 & 2121 \\
\hline & 1010 & 1011 & $102 n$ & 1011 & 1010 & 1021 \\
\hline & 0210 & 0211 & $022 ?$ & $n 211$ & ก210 & 0221 \\
\hline & $222 n$ & 2221 & 2200 & 2221 & 2220 & $22 ? !$ \\
\hline & 1120 & 1121 & $110 n$ & 1121 & 1120 & 1101 \\
\hline & $0 \cap 20$ & 0021 & nחn & 0921 & $\cap \cap 20$ & nח) \\
\hline & 2911 & 2020 & 2021 & 2010 & $2 \cap 21$ & 2020 \\
\hline & 1211 & 1220 & 1221 & 1210 & 1221 & 1220 \\
\hline & 0111 & 0120 & $\cap 12\}$ & 0110 & 0121 & 0120 \\
\hline & 2121 & 2100 & 2101 & $212 n$ & 2101 & $21 \cap ?$ \\
\hline & 1021 & $10 n 0$ & $1 \cap 01$ & 1029 & 1091 & $100 n$ \\
\hline & 0221 & $02 \cap 0$ & 0271 & 0220 & $02 \div 1$ & $020 ?$ \\
\hline & 2201 & 2210 & 2211 & $22 \cap 0$ & 2211 & $2 ? 10$ \\
\hline & 1101 & 1110 & 1111 & 110 & 1111 & 1110 \\
\hline & 0001 & 0010 & 0011 & 0000 & 0011 & ก)ा \\
\hline
\end{tabular}


139.

\begin{tabular}{|c|c|c|c|c|c|c|}
\hline REPET & \multicolumn{3}{|c|}{$V \mid I$} & \multicolumn{3}{|c|}{$V \| 11$} \\
\hline \multirow[t]{19}{*}{ Blocos } & 1 & 2 & 3 & 1 & 2 & 3 \\
\hline & 2000 & $20 \cap 1$ & 2010 & 2001 & $20 n \pi$ & 2011 \\
\hline & 1200 & 1291 & 12.10 & $12 \cap 1$ & $120 ?$ & 1211 \\
\hline & 010 & 0101 & 0110 & 0101 & n) & 0111 \\
\hline & 2120 & 2121 & 2170 & 2121 & 2120 & 2101 \\
\hline & 1020 & 1021 & 1000 & 102.1 & $102 ?$ & $\operatorname{lnn1}$ \\
\hline & 0220 & 0221 & 0200 & 72.2 .1 & $022 \pi$ & $72 \cap 1$ \\
\hline & 2210 & 2211 & 22.20 & 2211 & $221 n$ & 2221 \\
\hline & 1110 & 1111 & 1120 & 1111 & 1110 & 1121 \\
\hline & 0010 & $0 \cap 11$ & $0 \cap 20$ & 0011 & 0910 & 0021 \\
\hline & 2011 & $2 \cap 20$ & $2 \cap 21$ & 2010 & $2 \cap 21$ & $2 \cap 2 \cap$ \\
\hline & 1211 & $122 ?$ & 1221 & 1210 & 1221 & 1220 \\
\hline & 0111 & $012 ?$ & 0121 & $011 ?$ & 0121 & 0120 \\
\hline & 2101 & $211 n$ & 2111 & 2100 & 2111 & 2110 \\
\hline & 1001 & 1010 & $1 \cap 11$ & $\operatorname{lnn}$ & 1011 & 1010 \\
\hline & $02 \cap 1$ & 0210 & 0211 & $020 n$ & 0211 & 02.10 \\
\hline & 2221 & 2200 & 2201 & $222 ?$ & 2201 & 2200 \\
\hline & 1121 & 1109 & 1101 & 1120 & 1101 & 1100 \\
\hline & 0021 & non? & $00 n$ & $n \cap 2 \cap$ & $0 \cap 01$ & 0000 \\
\hline
\end{tabular}


b) Blocos de seis unidades

Interações confundidas: $A R, A C, B C, A C C$, AOD, ACD, BCO. Informação relativa: $A B, A C, B C: 7 / 8$ ABn, $A C \cap, B C D: 5 / B$ $\operatorname{ABC}: 3 / 4$

\begin{tabular}{|c|c|c|c|c|c|c|c|c|c|}
\hline \multirow[b]{2}{*}{ Blocos } & \multicolumn{9}{|c|}{ PE:ETIANA I } \\
\hline & 1 & 2 & 3 & 4 & 5 & 6 & 7 & 8 & 9 \\
\hline & 2000 & 2001 & 22.10 & 2100 & 2101 & 2010 & $22 \cap n$ & 2201 & 2117 \\
\hline & 1110 & 1111 & 1020 & $121 ?$ & 1211 & 1120 & 1010 & $1 \cap 11$ & 1220 \\
\hline & 0220 & 0221 & חח & 0020 & $0 \cap 21$ & nan & 0120 & 10121 & 0000 \\
\hline & 2211 & 2120 & 2121 & 2011 & 2220 & 2221 & 2111 & $2 \cap 2 \cap$ & $2 \cap 21$ \\
\hline & $1 \cap 21$ & 1200 & 1201 & 1121 & 1000 & 1001 & 1221 & 1100 & 1101 \\
\hline & 0101 & 0010 & ווחח & 7201 & 0110 & 0111 & 0001 & 0210 & 1211 \\
\hline
\end{tabular}

\begin{tabular}{|c|c|c|c|c|c|c|c|c|c|}
\hline & \multicolumn{9}{|c|}{ REPETICAOO $\|$} \\
\hline Blocos & 1 & $?$ & 3 & $\stackrel{4}{4}$ & 5 & 6 & 7 & 8 & 9 \\
\hline & 2001 & 2000 & 2211 & 2101 & 2100 & 2011 & 2201 & 2200 & 2111 \\
\hline & 1111 & חוו1 & 1021 & 1211 & 1210 & 1121 & 1011 & $\ln 10$ & 1221 \\
\hline & 02.21 & 0220 & 1010 & $9 \cap 21$ & 0020 & 0201 & 012.1 & 0120 & 0001 \\
\hline & 2210 & 2121 & 2120 & 2010 & 2221 & 2220 & 2110 & 2021 & 2020 \\
\hline & 1020 & 1201 & 1200 & 1120 & 1001 & 1000 & 1220 & 1101 & $110 n$ \\
\hline & 0100 & 0011 & noln & 9201 & 7111 & 0110 & nono & $n 211$ & 0210 \\
\hline
\end{tabular}




\begin{tabular}{|c|c|c|c|c|c|c|c|c|c|}
\hline & \multicolumn{9}{|c|}{ REPETICÃ 111} \\
\hline \multirow[t]{7}{*}{ Blocos } & 1 & 2 & 3 & 4 & 5 & 6 & 7 & ? & 9 \\
\hline & $2 n 0 ?$ & $20 n 1$ & 2110 & 2179 & 2101 & 2217 & 2200 & 2201 & $2 \cap 10$ \\
\hline & 1120 & 1121 & $12 n ?$ & 1220 & 1221 & 1000 & $1 \cap 20$ & $1 \cap 21$ & 1100 \\
\hline & $021 \pi$ & 0211 & $0 \cap 20$ & ח & 0011 & 0120 & 0110 & 0111 & 0230 \\
\hline & 2111 & 2220 & 2221 & 2.211 & 2020 & $2 \cap 21$ & 2011 & 2120 & 2121 \\
\hline & 1201 & 1010 & 1011 & $\ln \cap 1$ & 1110 & 1111 & 1101 & 1210 & 1211 \\
\hline & 0021 & 0100 & 0101 & 0121 & 0200 & $02 \cap 1$ & $\cap 221$ & nก) & $0 \cap n 1$ \\
\hline
\end{tabular}

\begin{tabular}{|c|c|c|c|c|c|c|c|c|c|}
\hline \multirow[b]{2}{*}{ Blocos } & \multicolumn{9}{|c|}{ REPETIÇAOO IV } \\
\hline & 1 & 2 & 3 & 4 & 5 & 6 & 7 & $\beta$ & 2 \\
\hline & 2001 & 2000 & 2111 & 2101 & 2100 & $2 ? 11$ & 2201 & 2200 & 2011 \\
\hline & 1121 & 1120 & 1201 & 1221 & 1220 & $\ln 1$ & $\ln 21$ & 1020 & 1101 \\
\hline & 0211 & 1210 & 0021 & 0011 & 0010 & 0121 & 0111 & 0110 & 0221 \\
\hline & $211 ?$ & 2221 & $222 n$ & 2210 & 2021 & 2020 & 2010 & 2121 & $212 n$ \\
\hline & $12 n 0$ & 1011 & 1010 & 1000 & 1111 & 1110 & 1100 & 1211 & 1210 \\
\hline & $0 \cap 20$ & $\ln 101$ & $\ln 100$ & $012 n$ & 0201 & $02 n n$ & 0220 & $\ln 001$ & 0000 \\
\hline
\end{tabular}

\begin{tabular}{|c|c|c|c|c|c|c|c|c|c|}
\hline & \multicolumn{9}{|c|}{ REPETIÇAO V } \\
\hline Dlocos & 1 & 2 & 3 & 4 & 5 & 6 & 7 & 8 & 9 \\
\hline & 2000 & $2 \cap n 1$ & 2110 & $210 n$ & 2101 & 2217 & 2200 & 2201 & 2010 \\
\hline & 12.10 & 1211 & 1020 & $101 ?$ & 1011 & $112 n$ & 1117 & 11111 & 1220 \\
\hline & 0120 & 0121 & $02 n n$ & 0220 & 0221 & nonon & $\ln 20$ & 0021 & 0100 \\
\hline & 2111 & $222 \pi$ & 2221 & 2211 & $202 n$ & $2 \cap 21$ & 2011 & 2120 & 2121 \\
\hline & 1021 & 1100 & $11 \cap 1$ & 11121 & $12 n n$ & 1201 & 1221 & 1000 & 1001 \\
\hline & 0201 & 0010 & $0 \cap 111$ & $\operatorname{lng} 1$ & 0110 & 0111 & $n 101$ & 0210 & 211 \\
\hline
\end{tabular}




\begin{tabular}{|c|c|c|c|c|c|c|c|c|c|}
\hline \multirow[b]{2}{*}{ Blocos } & \multicolumn{9}{|c|}{ REPET IC $\tilde{A} O V I$} \\
\hline & 1 & 2 & 3 & 4 & 5 & 6 & 7 & 0 & 9 \\
\hline & $2 \cap \cap 1$ & 2000 & 2111 & $21 \cap 1$ & 2100 & 2211 & 2201 & $22 ? n$ & 2011 \\
\hline & 1211 & $121 n$ & 1021 & 1011 & 1010 & 1121 & 1111 & 1110 & 1221 \\
\hline & 0121 & 0120 & 201 & 0221 & 0220 & nก) & 0021 & $n \cap 20$ & 0101 \\
\hline & 2110 & 2221 & 2220 & 2210 & 2021 & 2020 & 2010 & 2121 & 2120 \\
\hline & 1020 & 1101 & 1100 & 1120 & 1201 & 1200 & 1220 & 1001 & 1000 \\
\hline & 0200 & 0011 & 0010 & 0000 & 0111 & 0110 & 0100 & 0211 & 0210 \\
\hline
\end{tabular}

\begin{tabular}{|c|c|c|c|c|c|c|c|c|c|}
\hline \multirow[b]{2}{*}{ Blocos } & \multicolumn{9}{|c|}{ REPETICSAO VII } \\
\hline & 1 & 2 & 3 & 4 & 5 & 6 & 7 & 8 & 9 \\
\hline & 2000 & 2001 & 22.10 & 2100 & 2101 & 2010 & 22,00 & 22.01 & 2110 \\
\hline & 1220 & 1221 & 1100 & 1020 & 1021 & 1200 & 1120 & 1121 & 1000 \\
\hline & 0110 & 0111 & 0020 & 0210 & 02.11 & 0120 & 0010 & 0011 & 0220 \\
\hline & 2211 & 2120 & 2121 & 2011 & 2220 & 2221 & 2111 & 2020 & 2021 \\
\hline & 1101 & 1010 & 1011 & 1201 & 1110 & 1111 & 1001 & 1210 & 1211 \\
\hline & 0021 & 0200 & 0201 & 0121 & 0000 & 0001 & 0221 & 0100 & 0101 \\
\hline
\end{tabular}

\begin{tabular}{|c|c|c|c|c|c|c|c|c|c|}
\hline \multirow[b]{2}{*}{ Blocos } & \multicolumn{9}{|c|}{ REPETICAO } \\
\hline & 1 & 2 & 3 & 4 & 5 & 6 & 7 & 8 & 9 \\
\hline & 2001 & 2000 & 2211 & 2101 & 2100 & 2011 & 2201 & 2200 & 2111 \\
\hline & 1221 & 1220 & 1101 & 1021 & 1020 & 1201 & 1121 & 1120 & 1001 \\
\hline & 111 & 0110 & $0 \cap 21$ & 0211 & 0210 & 0121 & 0011 & 0010 & 10221 \\
\hline & 2210 & 2121 & 2120 & 2010 & 2221 & 2220 & 2110 & 2021 & 2020 \\
\hline & 1100 & 1011 & 1010 & 1200 & 1111 & 1110 & 1000 & 12.11 & 11210 \\
\hline & $n \cap 2 n$ & 0201 & 7200 & 0120 & 0001 & Inחת & 0220 & 0101 & ninn \\
\hline
\end{tabular}




\section{DISCUSSÃO}

Neste item, apresenta-se algumas vantagens e desvantagens do processo de confundimento descrito neste trabalho, em relação aos mëtodos mais conhecidos, ou sejam, - quadro de contrastes, para a sërie $2^{n}$ e o processo de con fundimento atravës da geometria finita.

$$
\text { nä-se,a sequir, o quadro de contrastes do }
$$

fatorial $2^{2}$ :

\begin{tabular}{|c|c|c|c|c|}
\hline \multirow{2}{*}{$\begin{array}{l}\text { Efeitos } \\
\text { Fatoriais }\end{array}$} & \multicolumn{4}{|c|}{ tratamentos } \\
\hline & (1) & $a$ & $b$ & $a b$ \\
\hline$A$ & - & + & - & + \\
\hline$B$ & - & - & + & + \\
\hline A A & + & - & - & + \\
\hline
\end{tabular}


144.

Fste é construido colocando-se o sinal

no tratamento onde a letra do respectivo efeito princinal a parece e (-) em caso conträrio. O sinal da interação re sulta do produto dos sinais dos efeitos nrincinais correspondentes.

Mota-se que o quadro oossui $3 \times 4=12$ sinais, ou genericamente $\left(2^{n}-1\right)\left(2^{n} ;\right.$.

Para confundir-se a interação $A B$ divide-se a repetição em dois blocos, onde, em um deles, são distribuidos os tratamentos (1) e ah e no outro, a e h, ou seja, um com os tratamentos com sinal (+) e o outro com os de sinal (-). No caso do fatorial $2^{6}$, por exemplo, o quadro de contrastes terá $\left(2^{6}-1\right)\left(2^{6}\right)=4 n 32$ s inais e a subdi visão de uma repetição em apenas dois blocos, os tornam excessivamente grandes, cada um contendo 32 tratamentos. Pode - se subdividi-la em quatro blocos de 16 parcelas cada, confundindo-se três graus de liberdade, ou seja, três efeitos' fatoriais. Para tanto, escolhe-se duas interações, como por exemplo, ABCD e ADEF. Os tratamentos são distribuidos nos ' quatro blocos de conformidade com os pares de sinais atri buidos a eles, nos contrastes referentes às duas interações consideradas $(++,+-,-\dot{0}--)$. A terceira interação que re sulta confundida ë denominada de interacão generalizada das duas primeiras e è obtida efetuando-se o produto das letras el iminando-se as que anarecem duas vezes. 
LOgO $(A B C \cap)(A B E F)=$ AABBCDFF = CDFF. neve-se tomar o cuidado de, ao escolher-se duas interações, não confundir efeitos' princinais ou interações de interesse.

Seguindo a mesma renra, pode-se formar oito blocos de oito tratamentos, tomando-se mais uma interação, " por exemplo, ACE. Procura-se no quadro de contrastes os tratamentos com os sinais +++, ++-, +-+, +--, -++, -+-, --+ e -- , que constituirão os oito blocos, semnre levando-se em conta a ordem dos sinais. Além das três interações anterio res, ABCD, ABEF e CnFF, resultam confundidas mais quatro,que são:

$$
\begin{aligned}
& (A B C D)(A C E)=B D F, \\
& (A B F F)(A C E)=B C F \\
& (A B C D)(A B E F)(A C E)=A \cap F .
\end{aligned}
$$

Como se rode verificar, na sērie $2^{n}$, com o au mento do nümero de fatores, o nrocesso de confundimento torna-se mais trabalhoso, quer nela construção do quadro de con trastes, quer pela escolha conveniente das interações a se rem confundidas. Por outro lado, o quadro de contrastes não é aplicável a fatoriais cujos fatores onssuam mais de dois niveis.

A vantanem do processo descrito no item 4 ., eे que a pröpria construção das tabelas bäsicas, para um determinado efeito fatorial, já seleciona os tratamentos com sinal (+) e (-) e a obtenção dos tratamentos com essas sinais, 
146.

para uma determinada interação, independe dos sinais dos res pectivos efeitos principais, o que torna o processo. menos trabal hoso.

0 processo de geometria finita tem a vantagem, sobre o processo descrito, de confundir diretamente as interaçōes. Assim, por exemnlo, o confundiment? da interação $A B C$ do fatorial $3^{3}$, é feito da seguinte forma, através dos qua tro comnonentes dessa interação:

$$
\begin{aligned}
& A \cap C \simeq A^{2} B^{2} C^{2} \\
& A B C^{2} \simeq A^{2} B^{2} C \\
& A B^{2} C \simeq A^{2} B C^{2} \\
& A B^{2} C^{2} \simeq A^{2} B C
\end{aligned}
$$

unde cada par contèm dois graus de liberdade. ne cada par, forma-se uma equação e cada equação determina os hlocos de uma repetição. A equação proveniente de $A \cap C^{2}$, por exemplo, é a seguinte:

$$
x_{1}+x_{2}+2 x_{3}=0,1,2(\operatorname{mó} d .3)
$$

donde resulta a mesma renetição se fosse usada a equação:

$$
2 x_{1}+2 x_{2}+x_{3}=n, 1,2 \text { (möd. 3) }
$$

formada a partir de $A^{2} B^{2} C$.

Para a formação dos hlocos, suhstitui-se o' primeiro, segundo e terceiro niveis, de cada uma das 27 combi nações de tratamentos, em $x_{1}, x_{2}$ e $x_{3}$, respectivamente. A soma destes, na equação, corresnonderá aos valores 0,1 e 2 , de acordo com a seguinte codificação: 


$$
\begin{array}{llll}
0 \simeq 0 & 3 \simeq 0 & 6 \simeq 0 & 9 \simeq n \\
1 \simeq 1 & 4 \simeq 1 & 7 \simeq 1 & 10 \simeq 1 \\
2 \simeq 2 & 5 \simeq 2 & 8 \simeq 2 & 11 \simeq 2
\end{array}
$$

os tratamentos cuja soma è zero, formam o pri meiro bloco; soma 1 , o senundo e soma 2, o terceiro bloco da repetição. Assim, por exemplo, a combinação nl? substituida' na equação $x_{1}+x_{2}+2 x_{3}$ seră:

$$
n+1+2.2=5 \simeq 2 \text {. }
$$

Para o confundimento da sërie $3^{n}$, atravès do processo do item 4., necessita-se primeiramente construir as tabelas básicas de um efeito orincinal e a partir destas,for mar as tabelas das interações de primeira, sęunta, etc.,ordens, até o confundimento da interação de interesse. Isto' torna o nrocesso mais trabalhoso quando comparado com o da geometria finita.

Entretanto, o nrocesso de geometria finita tem a desvantagem de só ser anlicävel aos fatorial $n^{n}$, sendo D necessariamente primo e não ser anlicäyel às sëries mistas. Com relação an nrocesso de ohtenção da informação relativa, descrito no item 4.6 , deste trabalho, tem-se como desvantagem, a necessidade de comoutador, nois ë necessārio a inversão de uma matriz, $M$, de ordem iqual ao nümero' de tratamentos. Para o comoutador $13 M-113 n$, o processo é vagaroso quando se trata de grandes fatoriais, como nor exem - 
148.

plo, o fatorial $3^{4}$, onde a obtenção da informação relativa, de um conjunto balanceado, necessita de aproximadamente 12 horas de processamento. Alèm disso, um efeito fatorial não pode estar completamente confundido, nois neste caso, a ma triz M não è inversivel, isto è, a matriz c è de ordem menor que $\underline{v}-1$, onde $\underline{v}$ é o nümero de tratamentos. Sendo $M$ não inversivel, não é nossivel obter-se a informação relativa por este processo. 


\section{CONCLUSÕES}

O processo de confundimento, descrito neste trahalho, nermite que se formulem as seguintes conclusões, consideradas mais importantes:

7.1. E possivel sistematizar o confundimento dos experimen tos fatoriais atrayès de um processo ünico, ou seja, - da formação de tabelas băsicas, para qualquer fatorial cujos fatores possuam dois ou três niveis.

7.2. Para a sërie $2^{n}$, pode-se confundir sempre as intera ções de maior ordem em função do nümero de blocos e do nümero de fatores, atravēs das regras citadas em 4.1.1., tornando o confundimento, consequentemente, e ficiente.

7.3. O processo não requer călculos matemäticos ou conhecí 
mentos teóricos profundos, sendo, essencialmente, um processo prätico.

7.4.0 processo possibilita, para um determinado fatorial, a subdivisão dos blocos em diversos tamanhos, desde' que o nümero de parcelas nor bloco seja mültiplo do nümero de niveis de, pelo menos, um dos fatores.

7.5.0s efeitos fatoriais que resultam confundidos são sem pre os de maior ordem possivel, para qualquer fatoria 1 .

7.6.0 conjunto de todas as repetições que podem ser obtidas, no confundimento de um determinado fatorial, pelo processo das tabelas básicas, constituem o fatorial' balanceado.

7.7.n processo de ohtenção da informação relativa è aplicável a qualquer fatorial, desde que nenhum dos efeitos esteja completamente confundido. 
151.

\section{SUMMARY}

A confounding procedure for factorials of' size $2^{n}$ is presented. Two properties of this procedure allow the confounding of factorials of any number of factors and levels.

Factorials with two and three levels (the series $2^{n}, 3^{n}, 3 \times 2^{n}, 3^{2} \times 2^{n}$ and $3^{3} \times 2^{n}$ ) were studied.

For each factorial of the $2^{n}$ series, un to $2^{5}$, balanced sets were constructed dividing the replicates into two blocks, up to blocks of two units each one, without confounding the main effects.

Balanced sets were constructed for the $3^{n}$ series $\left(3^{2}, 3^{3}\right.$ and $\left.3^{4}\right)$ with a division of the replicates into' three blocks for $3^{2}$, hlocks of nine and three units for $3^{3}$ 
152.

and blocks of twenty seven, nine and three units for $3^{4}$.

$$
\text { Factorials } 3 \times 2,3 \times 2^{2} \text { and } 3 \times 2^{3} \text {, of the }
$$

$3 \times 2^{n}$ series, $3^{2} \times 2$ and $3^{2} \times 2^{2}$ of the $3^{2} \times 2^{n}$ series

and $3^{3} \times 2$ of the $3^{3} \times 2^{\text {n }}$ series were studied under the general term of "mixed series".

A process for obtaining relative informati-

on based on the theory of incomplete blocks and the rene val theory of analysis of variance is also presented. 
9. IITERATURA CITADA

COCHRAN, W.G. e G.M. COX, 1960. Experimental Designs.2a.edição. Nova York, John Wiley E Sons, Inc.560 p.

FINNEY, D.J., 1947. The Construction of Confounded Arrange ments. Empire Journal Experimental Agricultural, 15: $107-112$

KEMPTHORNE, 0., 1952. The Design and Analysis of Experiments. Nova York, John Wiley E Sons, Inc. $631 \mathrm{p}$.

YATES, F., 1937. The Design and Analysis of Factorial Experiments. Bucks, Inglaterra, Commonwealth Agricultural Bureaux Farnham Royal. 95 p. (Technical Communication n:. 35 of the Commonwealth Bureau of Soils, Harpenden, Inglaterra). 
154.

10. APENDICE 1 
Instruções para o uso तo programa de obtenção da informação relativa

n nroorama, em linguagem FoRTRAN, nara o com putador 13M-1130, permite a obtenção da informação relativa dos efeitos fatoriais e é auxiliado nela subrotina PROD, a qual efetua o cálculo das variâncias dos niveis de cadá val dos nivels dos efeitos fatoriais.

A listanem do nrograma da suhrotina, bem como um exemplo de anlicação para o fatorial $3 \times 2$, encon tram-se no APENDICE 2 e o mesmo está programado para fatori ais que possuam, no máximo, cinco fatores, 100 tratamentos, inn blocos ou inn narcelas nor bloco.

Para qualq̧uer fatorial dentro desses limites, - programa calcula:
a) a matriz C;
b) a matriz $M$;
c) a matriz $\mathrm{M}^{-1}$
d) o produto $\mathrm{MM}^{-1}$; ontativo;
e) a matriz de dispersão:
f) as variàncias dos niveis de cada efeito

fatorial, considerando o confundimento;

g) as variancias dos niveis de cada efeito fatorial, não considerando o confundimento, e

h) a informação relativa. 
o produto $M M^{-1}$ é optativo, sendo executado ou não, de acordo com a entrada de dados e sö é necessärio quan do se tiver düvidas que a matriz $M$ seja singular. Se $M$ for singular, então existe pelo menos um efeito fatorial completamente confundido e do oroduto $M M^{-1}$ não resulta a matriz identidade.

Nas variâncias das interações, devem ser subtraidas ou somadas as variancias dos efeitos fatoriais, de acordo com o item 4.6. Assim, nara a interação An do exemplo dado no APENDICE 2, deve-se calcular:

- Variância com confundimento:

$$
V(A B)=(0,373611-0,111111-0,0625) \sigma^{2}=0,200 \sigma^{2}
$$

- Variância sem confundimento:

$$
\begin{gathered}
V(A B)=(n, 277777-0,111111-0,055555) \sigma^{2}=0,111111 \sigma^{2} \\
\text { Inf.Relativa } \approx-\frac{0,111111 \sigma^{2}}{0,20 \sigma^{2}}=0,55555=5 / 9
\end{gathered}
$$

Para o processamento do nrograma, os dados de verão ser prenarados de acordo com as instrucões de leitura, assim estabelecidas:

1a. leitura: I cartão: colunas de 1 a $80:$ Camno reservado pa ra a identificação do fatorial a ser orccessado ou qualquer outro comentärio. (Variävel XMOME). 2a. leitura: I cartão: colunas de 1 a 5 : Nümero de fatores ' 
do fatorial,com o ültimo algarismo na coluna 5. (Constante $M F)$.

Colunas 6 a in: nümero de repetições. Oltimo al Sarismo na coluna 10 . (Constante NR).

Colunas 11 a 15: número de blocos. Oltimo aloarismo na coluna 15. (Constante Mo).

Colunas if a 20: nümero de tratamentos por blo* co. N1timo algarismo na coluna 20.

(Constante NK).

Coluna 25: $M C=1$ : escreve a matriz C.

$$
N C=n \text {; não escreve. }
$$

Coluna 30: NM = 1: escreve a matriz $M$. Mุ $M=n:$ não escreve.

Coluna 35: Hny $=1$ : escreve a matriz $\mathrm{M}^{-1}$. MINV = n: não escreve.

Coluna 4n: MMI $=1$ : efetua o produto $\mathrm{MM}^{-1}$. MMI = ก: não efetua o produto.

Coluna 45: $M D=1$ : escreve a matriz de dispersão. $M D=n:$ não escreve.

4a. leitura: dispõe-se, em cada cartão, os tratamentos de de cada bloco de 5 em 5 colunas, em nümeros inteiros. 0 ültimo nivel de cada tratamento deverä ocupar a ültima das 5 cinco colunas. Não é necessärio ordenar os tratamentos. Na pägina seguinte ë dado um exemplo de codi ficaçãs, para o caso do fatorial $3 \times 2$. 
NUCLEO DE PROCESSAAENTO DE DADOS - UFPEL

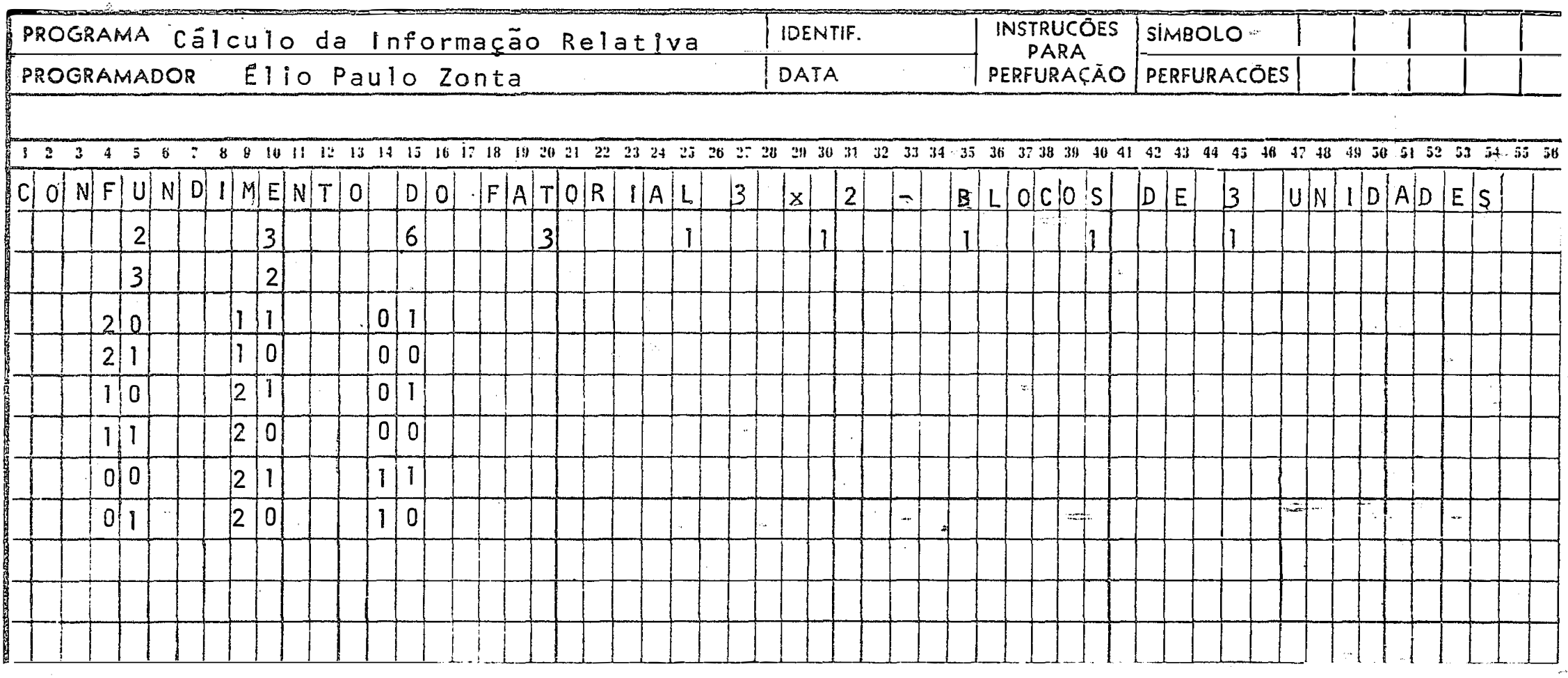

ESOUEMA PARA PEMEHAACA:

PARA

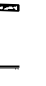


159.

11. APÊNDICE 2 
1. Iistagem do programa

PAGE 1

I/ JOB 2CAE ICAE

LOG DRIVE CART SPEC CART AVAIL PHY DRIVE 0000 0001 2CAE 2CAE $\quad 0002$ ICAE $\quad 0001$ 3CAE $\quad 0003$ 4CAE $\quad 0004$ SCAE $\quad 005$

V2 MI2 ACTUAL $16 \mathrm{~K}$ CONFIG $16 \mathrm{~K}$

*EQUAT (PRNTZ, PRNZ)

// DUP

*DELETE ARQO5

CART ID 2CAE DB ADDR 3EEO DB CNT $12 C 0$

* DELETE AREOG

CART ID 2CAE DB ADDR 3EED DB CNT 0960

* StOREdata WS Ua ARQ05 300

CART ID 2CAE DB ADDR 3EFO OB CNT $12 C 0$

* storedata la ua arqog 150

CART ID 2CAE DB ADDR $51 A D$ DB CNT 0960

// FOR

*I OCS (CARD,1132 PR INT ER, DISK, TYPEWR IT ER, KEYBOARD)

* LIST SOURCE PR OGRAM

* DNE WORD INTEGERS

* EXT ENDED PRECISION

C

C * $\quad$ C

C * PROGRAMA PARA A OBT ENGAO DA INFORMACAO RE LA TI VA Z

C * *

C * NOS EXPERIMENTOS FATORIAIS CONFUNOIOOS *

C *

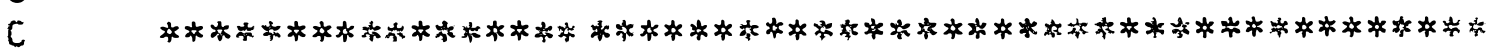

C PROGRAMAOOR - EL IO PAULO ZONTA

DIMENS ION XNOMEI 20), NNI V( 5$), N$ TP B (150), C MA TR 1300$)$, NCT $(150)$, XMATR130 10), NO(5), VMATR(300), ZMATR(150), AB(9)

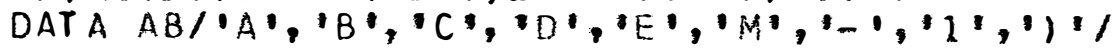

DEFINE FILE $5(300,200,0, K L)$

DEFINE FILE $6(300,100, U, K L)$ 
$1 \operatorname{READ}(2,2)(X N O M E(J), J=1,20)$

2 FORMAT $(20 A 4)$ WRITE $(3,3)(X$ NOME $(J), j=1,20)$

3 FORMATI'1',T $21,20 A 4,1 / 1$ WRITE $(3,153)$

READ $(2,4) N F, N R, N B, N K, N C, N M, N I N V, M M I, M D$

4 FORMAT (16I5) WRITE $(3,7) N F, N R, N B, N K$

7 FORMATIT21, 'FATORES $=113,2 X, 1$ REPETICOES $=1,13,2 X, 1$ BL OCOS = 1, I3, $2 x$, L'TAMANHO DOS BLOCOS $=1$, I3)

$\operatorname{READ}(2,4)(N N I V(J), J=1, N F)$ WRITE $(3,8)($ NNIV $(J), J=1, N F)$

8 FORMAT!T21: 'NIVEIS DOS FATORES $=1,2 x, 16151$

FIND $(5 \cdot 1)$

$N B P R=1 . *(N B / N R)+0.5$

$N T=N B P R+N K$

$N T 4=N T+1$

NT $2=2 \div N T$

$N T 3=N T+N K$

$K=1$

OO $10 \mathrm{~J}=1$, NF

$\operatorname{READ}(2,4)($ NTPB $(1,1, L=1$, NK $)$

$K T=1$

$K V=16$

IF $(N K-K V) 16,16,17$

$16 K V=N K$

17 WRITE $(3,9)(N T P B(L), L=K T, K V)$

9 FORMAT!T?:\&1(6)6)

IF (NK-KV) 60:60,61

$61 K T=K T+16$

$K V=K V+16$

IF $(N K-K V) 16,16,17$

$60005 \quad M 1=1$, NT2

5 CMATR $(M X)=0.0$

DO $6 \mathrm{M}=1, \mathrm{NK}$

$L U=M+N T$

$6 C M A T R(L U)=1$. $N A P B(H)$

WIRI TE $(5, K)(C M A T R(L), L=1$, NT 2$)$

$10 \mathrm{~K}=\mathrm{K}+3$

WRITE $(3,153)$

FIND(5II)

ORDENACAO DOS TRATAMENTOS

$\mathrm{I}=0$

$K B=1$

DO $11 \mathrm{~J}=1$, NBPR

READ(5,KB) (CMATR(L), L $=1, N T 2)$

$K B=K B+3$

OD $19 \quad J 6=1$, NK

$K A=N T+J \epsilon_{0}$ 
$19 \operatorname{CMATR}(J 6)=\operatorname{CMATR}(K A)$

DO $11 K=1$, AK

$1=1+1$

11 NTPB $(1)=$ CMATR $(K)+0.5$

DO $14 \quad I=1, N T$

DO $14 \mathrm{~J}=1, \mathrm{NT}$

I $F(J-I) 14,14,12$

$12 I F(N T P B(1)-N T P B(J)) 14,14,13$

13 TEMP $=$ NTPB (I)

$N T P B(I)=N T P B(J)$

$N T P B(J)=T E M P$

14 CONTINUE

DO $15 \quad 1=1$, NT

15 NCT $(1)=$ NTPB(I)

C CONSTRUCAO DAS MATRIZES C E M

FIND $(5 \cdot 1)$

$K B=1$

DO $65 \quad I=1, N B$

REAO (5'KO) (VMATR(JJ), JJ $=1, N T 2)$

$0066 \mathrm{~J}=1$, NK

$L U=N T+J$

66 NTPG (J)=VMATR $(L U)+0.5$

DO $68 M=1, N T$

$68 \operatorname{CMATR}(M)=0.0$

$0067 \mathrm{~K}=1$, NK

D $70 \mathrm{~L}=1, \mathrm{NT}$

IF (NTPB $(K)-N C T(L)) 70,69,70$

69 CNATR(L) $=1$.

GO TO 67

70 CONTINUE

67 CONT INUE

WRITE $(5 \cdot K B)(C M A T R(J J), J J=1$, NT )

$65 K B=K B+3$

$F I N O(5 \cdot 1)$

$K B=1$

DO $71 \quad I=1, N T$

$K A=1$

DO $72 \mathrm{~J}=1, N B$

$\operatorname{REAC}(5, K A)(C M A T R(J J), J J=1, N T)$

$X M A T R(J)=C M A T R(1)$

$72 K A=K A+3$

WRITE $\left(6^{\circ} K B\right)(X M A T R(J J), J J=1, N B)$

$71 K B=K B+3$

$F\left(N D\left\{\sigma^{\prime} 1\right\}\right.$

$K A=1$

DO $73 \quad 1=1$, NT

$D 076 \mathrm{~L}=1, N T$

76 VMATR $(L)=0.0$

READ $(6 \cdot \mathrm{KA})(C M A T R(\mathrm{JJ}), \mathrm{JJ}=1, N E)$ 
$K 8=1$

DO $74 \mathrm{~J}=1, N T$

REAO $(6 \cdot K B)(X M A T R(J \mathrm{~J}), \mathrm{JJ}=1, N B)$

DO $75 \mathrm{~K}=1$, NB

$75 \operatorname{VMATR}(J)=\operatorname{VMATR}(J)+X M A T R(K) * \operatorname{CMATR}(K)$

$74 K B=K . B+3$

$F I N D(5 \cdot K A)$

DO $77 M=1, N T$

$77 \operatorname{VMATR}(M)=-\operatorname{VMATR}(M) / F L O A T(N K)$

VMATR(I) = FLOAT (NR* $(N K-1)) / F L O A T(N K)$

WRITE (5'KA) (VMATR $(J \mathrm{~J}), \mathrm{JJ}=1, N T)$

FIND $\left(6^{\circ} K A\right)$

$73 K A=K A+3$

FIND(5'1)

$\mathrm{KA}=1$

DO $78 \quad I=1$, NT

READ ( $\left.5^{\circ} \mathrm{KA}\right)(C M A T R(\mathrm{JJ}), \mathrm{JJ}=1$, NT)

WRITE $\left(6^{\circ} \mathrm{KA}\right)(C M A T R(\mathrm{JJ}), \mathrm{JJ}=1, N T)$

FIND(5'KA)

$1 F(I-1) 81,79,81$

79 DO $80 \quad 11=2$, NT

$T 3=A B S(C M A T R(I I))+0.999$

$K 3=T^{3}$

$B C=A B S(C M A T R(I I))$

IF $(K 3-0) 81,80,81$

80 CONT I NUE

81 DO $82 \mathrm{~J}=1$, NT

82 CMATR $(\mathrm{J})=C M A T R(\mathrm{~J})+B C$

WRITE (5'KA) (CMATR (JJ), JJ $=1$, NT)

$78 K A=K A+3$

$F I N D(5 ' 1)$

$K A=1$

$00 \quad 87 \quad 1=1, N T$

READ (5'KA) (CMATK $(K), K=1, N T)$

DO $80 \mathrm{~J}=\mathrm{NT} 4, \mathrm{NT} 2$

88 CMATR(J) $=0.0$

$L U=I+N T$

CMATR $(L U)=1.0$

WRITE(5'KA) (CMATR $(K), K=1$, NTZ)

$87 K A=K A+3$

$N J=0$

IF $(10 C-1) 100,18,18$

18 FIND(6!1)

WRITE $(3,89) \triangle B(3)$

89 FORMATI//,T57, MA $T R I 21,4 X, 4 A 1, / 1$

$90 \mathrm{KA}=1$

DO $99 \quad I=1$, NT

If $(N J-1) 91,92,93$

91 RेEAD(6'KA) XMATRIJJ $/, J J=1, N T)$ 
601094

$92 \operatorname{RFAD}\left(5^{\circ} \mathrm{KA}\right)(X M A T R(J J), J J=1, N T)$

GO $\mathrm{rO} 94$

$93 R E A D(5, K A)(X M A T R(J J), J J=1, N T 2)$

DO $50 \mathrm{~J}=1$, NT

$L U=J+N T$

$50 \times \operatorname{MATR}(J)=\times \operatorname{MATR}(L U)$

$94 \mathrm{KT}=1$

$K V=9$

IF $(N T-K V) 95,95,96$

$95 K V=N T$

96 WRITE $(3,97) \mathrm{I},(X M A T R(J J), J J=K T, K V)$

97 FOR MAT (IX, 'LINHA', I 4, 2X, 9F12.7)

I $F(N T-K V) 99,99,98$

$98 K T=K T+9$

$K V=K V+9$

IF (NT-KV) 95,95,96

$99 K A=K A+3$

100 IF $(N J-1) 101,103,114$

101 IF $\left(N H_{0}=1\right) 103,102,10 ?$

10? FINO(5'1)

$N J=1$

WRITE $(3,89) \triangle B(6)$

GO TO 90

C INVFRSAO DA MATRIZ M

$103 \mathrm{NJ}=1$

$K A=1$

$00129 \quad 1=1, N T$

READ(S'KA) (CMATR $(M), M=1, N T Z)$

$K B=1$

DO $110 \mathrm{~J}=1$, NT

I $F(K A-K B) 104,110,104$

104 REAO(5 KB) (XMATR (M), M=1, NT2)

$A 1=C M A T R(I)$

$A 2=X M A T R(I)$

$K 2=A 2 *(10 . * 7)+0.5$

I $F(K 2-0) 105,110,105$

$105 K 1=A 1 *(10 . * 7)+0.5$

IF $(K 1-0) 108,106,108$

106 DO $107 \mathrm{~L}=\mathrm{I}, \mathrm{NT}$ ?

$107 \mathrm{CMATR}(L)=C M A T R(L)+X M A T R(L)$

WRIII (5,KA) (CMATR $(M), M=1$, NT2)

$A L=A ?$

108 OO $109 \mathrm{~K}=1$, NTZ

$109 \times \operatorname{XMAT}(K)=X M A T R(K)-1$ A2 $* \operatorname{CMATR}(K)) / A 1$

WRITE (5,KB) (XMATR (M), $M=1, N T 2)$

$110 K B=K B+3$

$129 K A=K A+3$

$K A=1$

$00113 I=1$, NT

READ(5'KA) (CMATR $(M), B=1, N T 2)$ 
$B B=C M A T R(I)$

DO $112 J=1, N T 2$

112 CMATR(J) =CMATR(J)/BB

WRITE $(5 \cdot K A)(C M A T R(M), M=1, N T 2)$

$113 K A=K A+3$

FIND(5'1)

$K B=1$

DO $270 \quad I=N T 4, N T 2$

$K A=1$

DO $271 \mathrm{~J}=1$, NT

$\operatorname{READ}(5: K A)(C M A T R(J \mathrm{~J}), J J=1, N T 2)$

$\operatorname{VMATR}(J)=$ CMATR(I)

$271 K A=K A+3$

WRITE $(5 \cdot K A)(V M A T R(J \downarrow)\rfloor J=1,, N T)$

$270 \quad K B=K B+3$

114 IF (NJ-1)115,115,117

$1151 F(N I N V-1) 117,116,116$

$116 \mathrm{~F}(\mathrm{ND})\left(5^{\prime} 1\right)$

$N J=$ ?

WRITE $(3,89), A E(6), A B(7), A B(8)$

GO TO 90

C PRODUTO DAS MATKIZES M E $M-1$

117 IF $(M M 1-1) 501,118,118$

118 FINDIS: 11

WRIT $(3,89) A B(6), A B(6), \triangle B(7), A B(8)$

$K A=1$

DO $125 \quad 1=1$, NT

$\operatorname{READ}(6 \cdot K A)(C M A T R(M), M=1, N T)$

DO 51 I $K=1$, NT

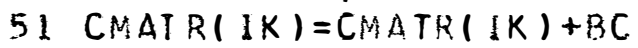

$F I N O(5,1)$

$K B=1$

DO $121 \mathrm{~J}=1, \mathrm{NT}$

$X M A T R(J)=0.0$

READ(5:KB) (VMATR(JJ), JJ $=1, N T)$

DO I19 K=I,NT

$119 \times \operatorname{MATR}(J)=X M A T R(J)+C M A T R(K) * \operatorname{VMATR}(K)$

$121 \mathrm{~KB}=\mathrm{KB}+3$

$F I N D(6 \cdot K A)$

$K T=1$

$K V=9$

$I F(N T-K V) 122,122,123$

$122 K V=N T$

123 WRITE $(3,97) I,(X M A T R(J \mathrm{~J}), \mathrm{JJ}=K T, K V)$

I $F(N T-K V) 125,125,124$

$124 K T=K T+9$

$K V=K V+9$

IF $(N T-K V) 122,122,123$

C

$125 K A=K A+3$

C calculo da matrit. de dispersao 


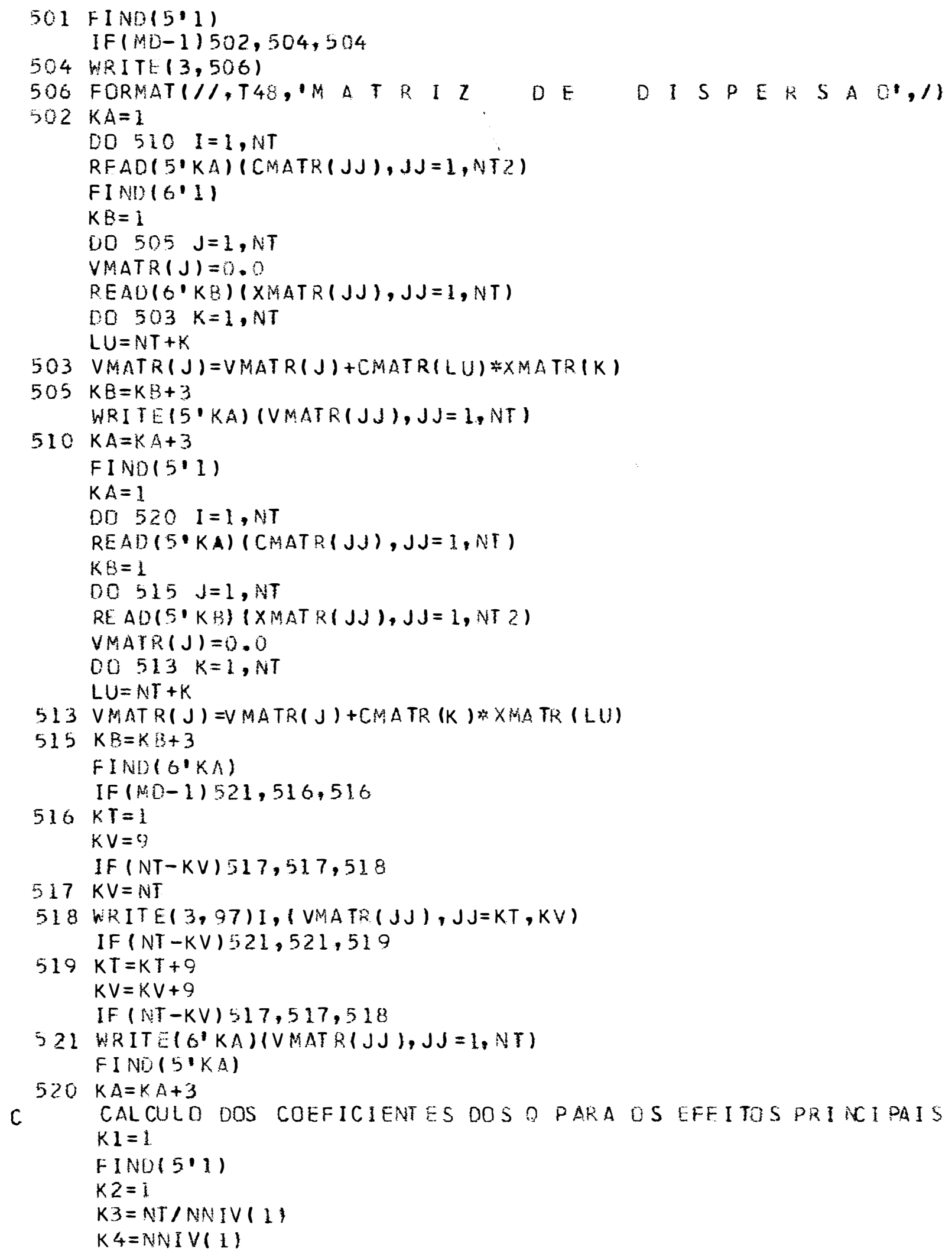

521 WRITE (6 KA) (VMATR(JJ),JJ $=1, N T)$

$F I N O)(S \cdot K A)$

$520 K A=K A+3$

C CALCULO DOS COEFICIENTES DOS 9 PARA OS EFFITOS PRI KCIPAIS $K I=1$

FINO(5.1)

$\times 2=1$

$K 3=$ NT/NNIVIII

$K 4=N N I V(1)$ 
DO $130 \quad I=1, K 4$

DO $127 \mathrm{~J}=1$, NT

$127 \operatorname{CMATR}(J)=0.0$

DC $128 K=K 2, K 3$

$128 \operatorname{CMATR}(K)=1$.

WRITE(S・Kl) (CMATR $(J J), J J=1$, NT)

$K 1=K 1+3$

$K 2=K 2+N T / N N I V(1)$

$130 K 3=K 3+N T / N N I V(1)$

$k 2=1$

$K 3=N T / N N I V(1)$

$K 5=N N I \forall(2)$

$K 6=K 3 / N N I V(2)$

$00135 \quad I=1, K 5$

DO $131 \mathrm{~J}=1, \mathrm{~K} 3$

$131 \operatorname{CMATR}(\mathrm{J})=0.0$

DO $132 K=K 2, K 6$

$132 \operatorname{CMATR}(K)=1$.

$K T=0$

DO $133 \mathrm{~L}=1, \mathrm{~K} 4$

DO $133 M=1, K 3$

$K 7=K 7+1$

$133 \times \operatorname{MATR}(K 7)=\operatorname{CMATR}(M)$

WRITE $(5 \cdot K l)(X M A T R(J J), J J=1, N T)$

$K I=K l+3$

$K 2=K 2+K 3 / N N I V(2)$

$135 K 6=K 6+K 3 / N N I V(?)$

IF $(N F-2) 151,151,136$

$136 K 2=1$

$K 6=K 3 / N N I V(2)$

$K 7=K 6 / N N I V(3)$

$K 8=$ NNIV $(3)$

OO $140 \quad 1=1, K 8$

$00137 \mathrm{~J}=1, \mathrm{~K} 6$

$137 \operatorname{CMATK}(\mathrm{J})=0.0$

DO $138 \quad K=K 2, K 7$

$138 \operatorname{CMATR}(K)=1.0$

$K 9=0$

DD $139 \quad L=1, K 4$

$00139 \quad M=1, K 5$

$00139 N=1, K 6$

$K 9=K 9+1$

$139 \times M A T R(K 9)=C M A T R(N)$

WRITF(5'KI) (XMATR(JJ), JJ $=1, N T)$

$\mathrm{K} l=\mathrm{K} l+3$

$K 2=K 2+K 6 / N N I V(3)$

$140 \quad K 7=K 7+K \epsilon_{3} / N N I V(3)$

IF $(N F-3) 151,151,141$

$141 \times 2=1$ 
DO $142 \mathrm{~J}=1, \mathrm{~K} 7$

$K T=K 6 / N N I V(3)$

$K 9=N N I V(4)$

$\mathrm{N} l=K 7 / \mathrm{NN} I V(4)$

DO $145 \quad I=1, K 9$

$142 \operatorname{CMATR}(\mathrm{J})=0.0$

DO $143 \mathrm{~K}=\mathrm{K} 2$, N1

$143 \operatorname{CMATR}(K)=1.0$

$\mathrm{N} 2=0$

N3 $=K 4 * K 5 * K 8$

DO $144 \mathrm{~N}=1, \mathrm{N3}$

DO 144 II $I=1, K 7$

$\mathrm{N} 2=\mathrm{N} 2+1$

144 XMATR(N2) = CMATR (II)

WRITE $\left(5^{\circ} K 1\right)(X M A T R(J \mathrm{~J}), \mathrm{JJ}=1, N T)$

$K 1=K 1+3$

$K 2=K 2+K 7 / N N I V(4)$

$145 \mathrm{NI}=\mathrm{NI}+K 7 / N N I V(4)$

IF $(N F-4) 151,151,146$

$146 \mathrm{~K} 2=1$

$N I=K 7 / N N I V(4)$

$N 2=N N I V(5)$

DO $150 \quad l=1, N 2$

$00147 \mathrm{~J}=1, \mathrm{~N} 1$

147 CMATR $(J)=0.0$

CMATR $(K 2)=1.0$

$N 4=0$

$\mathrm{N} 3=\mathrm{N} 3 * \mathrm{~K} 9$

DO $148 \quad K=1, N 3$

DO $148 \quad L=1, N 1$

$N 4=N 4+1$

148 XMATR $(N 4)=$ CMATR(1.)

WRITE $(5 \cdot K I)(X M A T R(J J), J J=1, N T)$

$K 1=K 1+3$

C

$150 \quad K 2=K 2+1$

CALCULO DAS VARIANCIAS E DA INFORMACAO RELATIVA

151 FIND(5.1)

WRITE $(3,152)$

152 FORMAT $1 / /$, T44, 'VARIANCIAS UOS EFEITUS FATURIAIS', WR ITE $(3,570)$

570 FOR MATIT47,' INTERACUES NAO CURRIGIDAS', )

WR ITE $(3,153)$

153 FORMAT $(T 20,82(1-1)$,

WRITE $(3,154)$

154 FORMATIT39, V VARIANCIA COM CONFUND.', $2 X$, VVARIANCIA SEM C ONF UNO.',3X 1, 'INF. RELATIVA', ) WRITE $(3,153)$

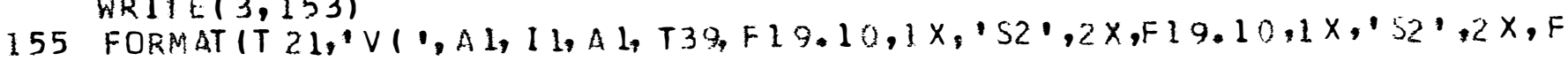
...114.19: 
156 FORMAT(T21,'V(',2(A1,I1),A1,T39,F19,10,1X,'S2', 2X,F19.10,1 $, 152 ', 2$ $1 \times, F 14.101$

157 FORMAT(T21,'V(',3(A1,11), A1,T39,F19,10,1X,'S2',2X,F19.10,1X,'S2',2 $1 X, F 14.101$

162 FORMAT(T21,'V1',4(A1,I1),A1,T39,F19.10,IX,'S2',2X,F19.10,1X,'S2', 2 $1 X, F 14.101$

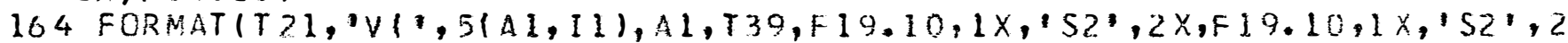
$1 X, F 14.101$

$M=1$

$A 1=0$.

$\Delta 2=0$

$A 3=0$.

$00160 \quad I=1$, NF

$K 2=N N I V(I)$

DO $160 \mathrm{~J}=1, \mathrm{~K} 2$

RE AO ( $5 \cdot M)(C M A T R(J J), J J=1, N T)$

CALL. PROE(CMATR, NT, NR, XMATR, NCT, VMATE, A 1,A2,A3)

$L=J-1$

IF $(L-0) 153,158,159$

158 NO(I) $=M$

159 WRITE $(3,155) A B(1), L, A B(9), A 1, A 2, A 3$

$160 \mathrm{M}:=M+3$

FIND (5'1)

DO $170 \quad I=1, N F$

$K I=N N I V(I)$

DO $169 \mathrm{~J}=1$, NF

IF(J-I) 169,169,161

$161 K \geq=\operatorname{NNIV(J)}$

DO $168 \mathrm{~K}=\mathrm{I}, \mathrm{KI}$

$\mathrm{NI}=\mathrm{NO}(\mathrm{I})+3 * \mathrm{~K}-3$

$11=K-1$

DO $268 \quad L=1, K 2$

$\mathrm{N} 2=\mathrm{NO}(\mathrm{J})+3 * \mathrm{~L}-3$

$12=L-1$

READ (5'NI) (CMATR(JJ), JJ $=1$, NT)

KEAO( $5 \cdot N Z)(X M A T R(J J), J J=1, N T)$

DO $163 \quad N=1$, NT

$163 \operatorname{CMATR}(N)=\operatorname{CMATR}(N): \operatorname{MATR}(N)$

CALL PRODICMATR, NT, NR, XMATR, NCT, VMA TR, AI, A 2,A3)

WRITE $(3,156) A B(1), 11, A B(J), I 2, A B(9), A 1, A 2, A 3$

168 CONT INUF

169 CONTINUE

170 CONTINUF.

IF $(N F-2) 260,26 \bullet 171$

$17100185 \quad 1=1, \mathrm{NF}$

$K I=N N I V(I)$

DO $184 \mathrm{~J}=1, \mathrm{NF}$

IF $(J-I) 184,184,172$ 
$172 K .2=\operatorname{NNIV}(J)$

DO $183 \mathrm{~K}=1$, NF

I $F(K-J) 183,183,173$

$173 K 3=N N I V(K)$

DO $182 \quad L=1, K 1$

$N I=N O(I)+3 * L-3$

$11=L-1$

DO $182 \quad M=1, K 2$

$N 2=N O(J)+3 * M-3$

$12=\mathrm{P}_{1}-1$

DO $182 \quad \mathrm{~N}=1, \mathrm{~K} 3$

$N 3=N O(K)+3 * N-3$

I $3=\mathrm{N}-1$

READ(5'N1)(CAATR (JJ), JJ $=1, N T)$

READ (5, NZ) (XMATR $(J J), J J=1, N T)$

READ(5'N3) (ZMATR (JJ), JJ $=1, N T)$

DO $177 M M=1$, NT

177 CMATR (MM) $=$ CMATR $(M M) \div X M A T R(M M) * Z M A T R(M M)$

CALL PRODICMATR,NT, NR, XMATR, NCT, VAATR,A I,A 2,A31

WRITE( 3, 157)AB(1), 11,AB(J), I2,AB(K), I3, AB(9),A1, A2, A3

182 CONTINUE

183 CONTINUE

184 CONTINUE

185 CONTINUE

IF $(N F-3) 260,260,186$

$186 \mathrm{DO} 218 \quad \mathrm{I}=1, \mathrm{NF}$

$K I=N N I V(1)$

$00217 \mathrm{~J}=1, \mathrm{NF}$

IF(J-1) $217,217,187$

$187 K 2=N N I V(J)$

DO $216 \mathrm{~K}=1$, NF

I $F(K-J) 216,216,188$

$188 K 3=N N I V(K)$

DO $215 \mathrm{~L}=1, \mathrm{NF}$

IF $(L-K) 215,215,189$

$189 K 4=N N I V(L)$

DO $214 M=1, K l$

$\mathrm{NI}=\mathrm{NO}(\mathrm{I})+3 * \mathrm{M}-3$

I $1=M-1$

D. $214 \mathrm{~N}=1, \mathrm{~K} 2$

$N Z=N O(J)+3 * N-3$

$12=N-1$

DO 214 II $=1, K 3$

$N 3=N O(K)+3 * I I-3$

$13=11-1$

DO $214 K K=1, K 4$

$N 4=N C(L)+3 * K K-3$

$14=K K-1$

READ(5:NI) (CMATR(JJ), JJ $=1, N T)$

READ (5, N2) (XMATR (JJ),JJ $=1, N T)$ 
READ (5'N3) (ZMATR (JJ),JJ $=1, N T)$

READ (5, N4) (VMATR(JJ), JJ $=1, N T)$

DO $190 \quad L L=1, N T$

190 CMATR (LL) =CMATR(LL) *XMATR(LL) *ZMATR(LL) *VMATR(LL)

CALL PROD (CMATR, NT, NR, XMATR, NCT, VMATR,A 1, A2,A3)

WRITE(3,162)AB(I), I1,AB(J), 12,AB(K), I3, AB(L), I4,AB(9),A 1, A $2, A 3$

214 CONTINUE

215 CONT INUE

216 CONT I NUE

217 CONT INUE

218 CONTINUE

I F (NF-4) 260,260,219

$219 K I=N N I V(1)$

$K 2=N N I V(2)$

$K 3=N N I V(3)$

$K 4=N N I V(4)$

$K 5=N N I V(3)$

$K 6=0$

DO $250 \quad 1=1, K 1$

$\mathrm{Nl}=\mathrm{NO}(1)+3 * 1-3$

$11=1-1$

DO $250 \mathrm{~J}=1, \mathrm{~K} 2$

$\mathrm{N} 2=\mathrm{NO}(2)+3 * \mathrm{~N}-3$

I $2=\mathrm{J}-1$

DO $250 \mathrm{~K}=1, \mathrm{~K} 3$

$N 3=N O(3)+3 * K-3$

$13=k-1$

DO $250 \quad L=1, K 4$

$N_{4}=\mathrm{NO}(4)+3 \div \mathrm{L}-3$

$14=L-1$

DO $250 \quad M=1, \mathrm{KS}$

$N 5=N O(5)+3 * M-3$

I $5=M-1$

DO $220 \quad \mathrm{~N}=1, \mathrm{NT}$

220 CMATR $(N)=0.0$

$K G=K 6+1$

CMATR $(K 6)=1.0$

CALL PROOICMATR, NT, NR, XMATR, NC T, VMATR, A1, A2, 1.31

WRITE $(3,164) A B(1), 11, A B(2), I 2, A B(3), 13, A B(4), 14, A B(5), I 5, A B(9), A 2$,

$1 A 2, A 3$

250 CONTINUE

260 HRITE(3,153)

GO TO 1

END

FEATURES SUPPORTED

ONE WCRD INTECERS

FXIENDED PRECISION 
CORE REQUIREMENTS FOR

COMMON O VARIABLES 3648 PROGRAM 5578

END OF CONPILATION

IIXEQ 1

*FILES (5, ARQO5), (6, ARQO6)

2. tistagem da subrotina PROD

$\begin{array}{lccc}11 \text { JOB } & \text { 2CAE ICAE } & & \\ \text { LOG DRIVE } & \text { CART SPEC } & \text { CART AVAIL } & \text { PHY DRIVE } \\ 0000 & \text { 2CAE } & \text { 2CAE } & 0002 \\ 0001 & \text { ICAE } & 1 C A E & 0001 \\ & & 3 C A E & 0003 \\ & & 4 C A E & 0004 \\ & & 5 C A E & 0005\end{array}$

V2 MIL ACTUAL $16 \mathrm{~K}$ CONFIG $16 \mathrm{~K}$

*EQUAT( PRNTZ, PRNZI)

// FOR

* IST SOURCE pROGRAM

*ONE WDRD INTEGERS

*EXTENDED PRECIS ION

SUBROUTI NF PRDDICMATR, NT, NR,XMATR, NCI, VMA TR, A 1, A $2, A 3\}$

DIMENS ION CMATR(300), XMATR (300), VMA TR (300), NC T (150)

FIND $(6 \cdot 11$

$L \mathrm{~L}=0$

DO $1 \mathrm{I}=1$, NT

NCT $(I)=C M A T R(1)+0.5$

$1 L I=L I+N C T(I)$

$K E=1$

DO $2 \quad I=1$, NT

READ (6.KE) (CMATR (JJ), JJ $=1, N T)$

$\operatorname{VMATR}(I)=0.0$

DO $3 \mathrm{~J}=1$, NT

XMATR(J)=FLOAT(UCT(J))/FLOAT(NR\#LI)

3 VMATRII $)=\operatorname{VMATR}(I)+C M A T R(J) * F L O A T(N C T(J))$

$2 K E=K E+3$

$A 1=0$. 
173.

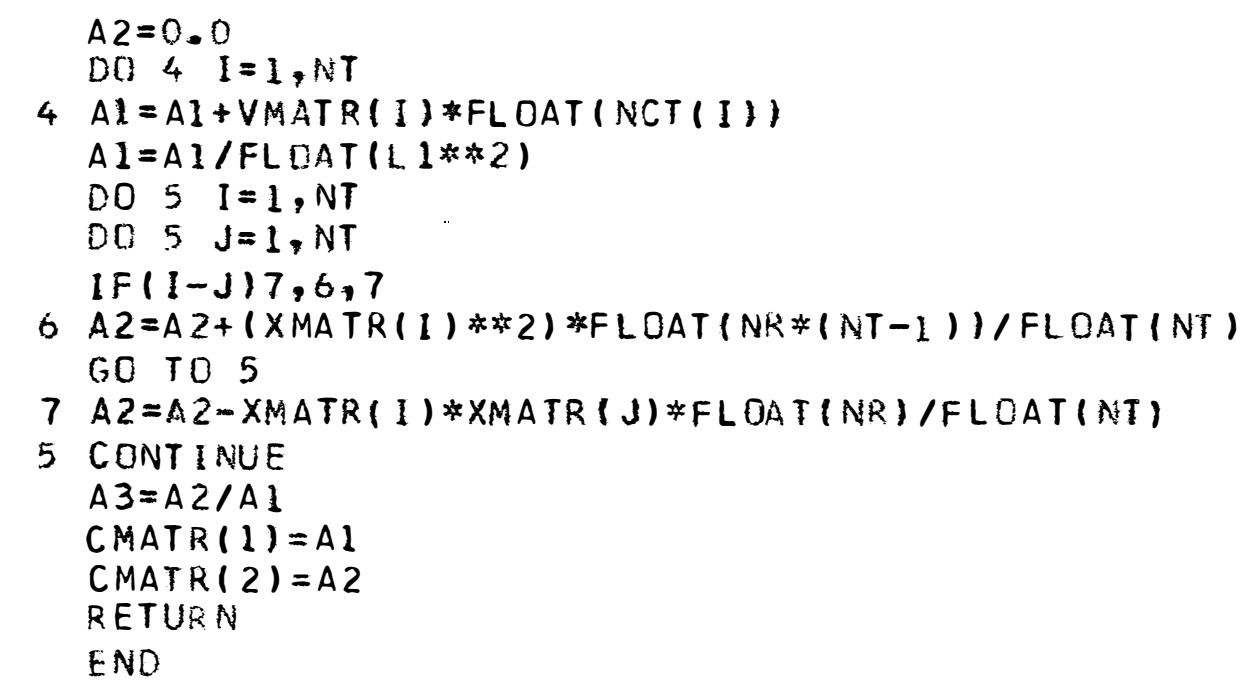

FEATURES SUPPORTED

ONE WORD INTEGERS

EXTENOED PRECISI ON

CORE REQUIREMENTS FOR PROO

$\begin{array}{lllll}\text { COMMON } & \text { O VARIABLES }\end{array}$

RELAT IVE ENT RY POINT ADDRESS IS 0010 (HEX)

ENO OF COMPILATION

$1 /$ DUP

* DELETE PROD ICAE

CART ID ICAE OB ADOR OI80 DB CNT OOIE

* STORe WS UA PROD ICAE

CART ID ICAE DB ADOR O180 DB CNT OOIE 


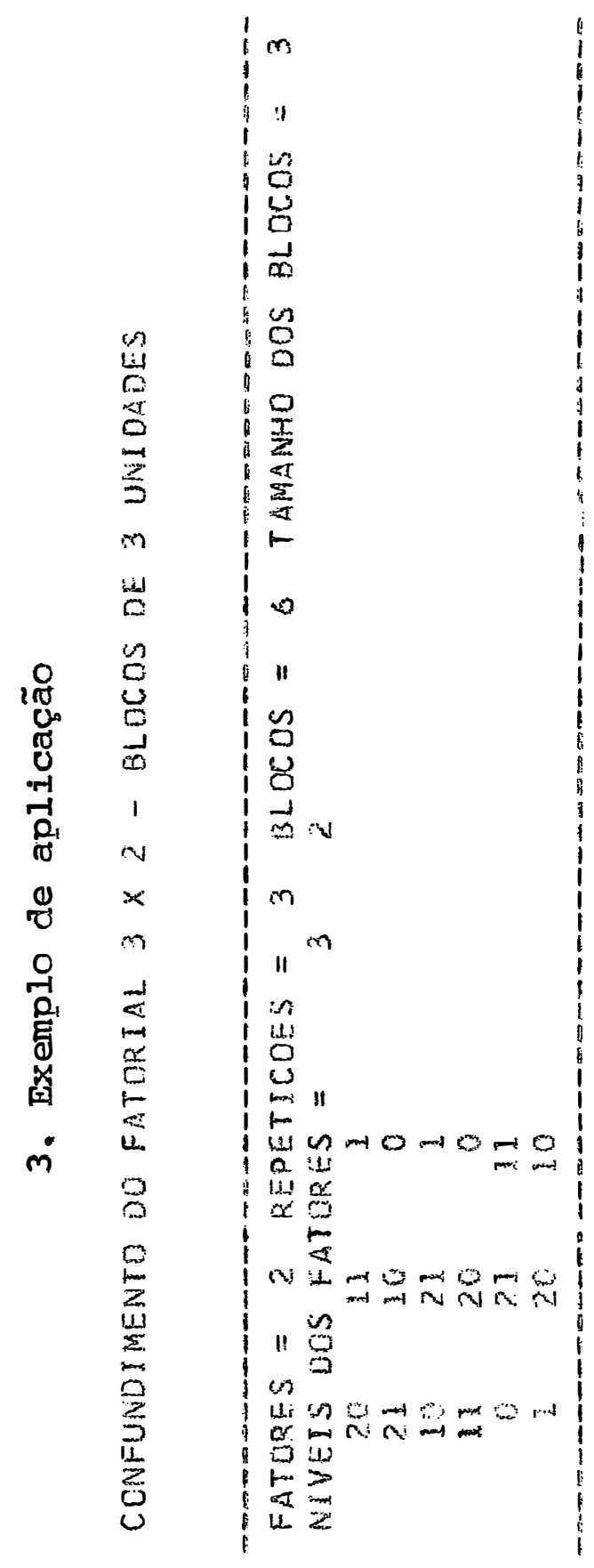

on 0 m om 0 m $0 m \omega m$ $0 m 0 m \approx 0$ $0 m \infty m \infty 0$ $0 \mathrm{mom} 0 \mathrm{~m}$ $0 m \omega m=0$

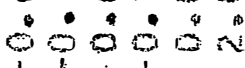

जm $m 0 m 000$ $m \infty m \circlearrowleft 0$ $m$ in 100 $m=000$

* $m$ o $m$ क

- 5000

$x$ iो 1 iा

Lm 30 n

ब 0 mos $0 \mathrm{~m}$ 0 m 00 ต

$50 \mathrm{~m} \omega 0 \mathrm{~m}$ $0 \mathrm{~mol} 0 \mathrm{~m}$ $\infty \mathrm{m} 00 \mathrm{~m}$ $0 \mathrm{mog}$ : 0 -

1

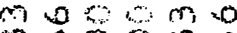
m $050 \mathrm{mo}$ $m 000 m$ $m w 00 m 0$ m $00 \mathrm{~m}$ m 000 n 0 $m 00=m \infty$

3000

10000

$00 \%$ क " w $0 \leqslant 0 m$ m $60 \mathrm{~m}$

4.9

करण

काष 0 \% का की $00 \mathrm{cos} \infty$ $0 \mathrm{~m} 0 \mathrm{mo}$ $\Leftrightarrow m \infty m$ \% m 0 m $0 \mathrm{mom} 0$

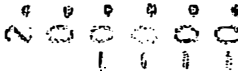

man $\mathrm{Nm}$ w?

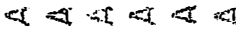
I I I I -

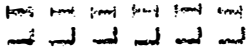

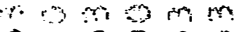
romo m $m s$ m $m$ $m o m o m m$ momo in $m o m o m$ $m D \mathrm{mom}$ $\because \dot{0} \dot{0} \dot{0}$

$20 m 0 \mathrm{~mm}$ $0 m 8 \mathrm{~mm}$ $\mathrm{Om} \mathrm{mm} \mathrm{m}$ \& $\mathrm{mm} m \mathrm{~m}$ $\approx m m m$ m

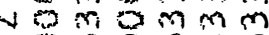
$s$ m $6 m m$ س

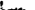

क 6 का मो \& $3 \mathrm{~m}$ in m $m 0 m m s$

$m m m m$ m $m$ in m $m$ $m, m m m=$ $m b m \pi$ a

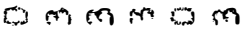
cos $m m m$ $5 m m \infty m$ $0 m m m \& m$ य $m$ m तn m $m$ $0 m \mathrm{~mm}$ - $: \circ:$ कर

लm m (1)

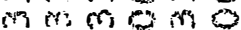
mi तो $m 0$ in m को $\mathrm{m}$ का हो ON

$m m<m o m$ लि $\mathrm{m} 5 \mathrm{~m} \mathrm{~m}$ $m m o m m m$ $m m \infty m$ जा ल० $0 \mathrm{~m}$ तथ $\mathrm{m}$ तो तो $95 \mathrm{~m}$ $0 \quad 0 \quad 80$ m

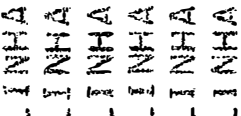

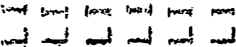




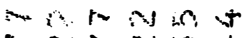
mar iv $N D N N$ t $N$ im $N$ a in tos 0.000 U $0<000$ $5 \operatorname{lol}^{\circ} 0 \mathrm{O}$ 00000 1

NNRnitin Nanitin

$N m n \in$ in

MNANOC o. $\operatorname{sic} 0$ in 000

पथ $0<00$

का मी

if

mos

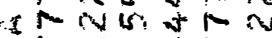

N N W $+N$ $2 N \cos N$ N is of co in in 0 o in 50

$000+50$

की

Nhytar Nint $\operatorname{tin}$ Ne $\tan N$ a me. OA on is o 0 o $00 \omega 000$ 0000

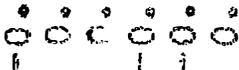

ind $4+R N R$ of m N W N wo his on 040000 0000

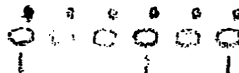
and viriene or $r N A n$ a ro 15 o (n) 3000 $\leftarrow \Leftrightarrow 0 \Leftrightarrow$

- If a

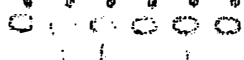

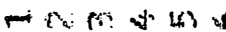

arie $x<x<x$

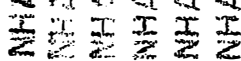

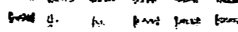

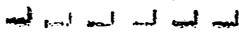

$60000 \%$ $00000 \%$ 00005 90500 000000 000000 00000

$00000=$

020065 005030 900000

N 0060 $\therefore 000$

i $100-0$

$m$

4

00000

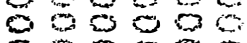

000000

2 $00 \mathrm{O}$

000006

020000

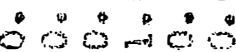

09000

020360

0000

000000

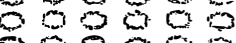

060200

का mi

wh in the in. in in in 00

(s)

in in in in $\infty$

$m 00 m \infty$

$x$

a) $m \infty \mathrm{m}$ क m $\rightarrow \sigma \rightarrow \sigma$ in

IS

$00 \mathrm{~cm}$

a.

is

- के

1.-

in in in in $\alpha \infty$

in in the in

a in in $\ln$ in $\rightarrow$ $00000 \mathrm{~m}$ त) $00 \mathrm{~m} m$ 0 an 0

W.

- .0 .0

m

\% अ

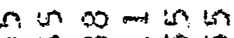
thin in 00 in in

$N$ in $n \infty \pi$ in $00 m$ oo EC $m m$ m

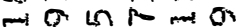
का m $\therefore 0^{\circ} \dot{0}^{\circ}$

$1 ; 11$

$\leftarrow$

జي.

in $n$ mat $\infty$ in th in in $-\infty$ in in in in $\rightarrow \infty \mathrm{kn}$ in $000 \mathrm{mos}$ $m$ os nल क o a n in a : की 00

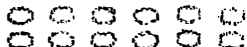
030000 000506

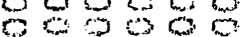
0000 00000

$c^{2}$ m

00000 50000 000000 $-\infty 000$ 0000 $\rightarrow 0000$

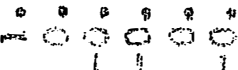

$\pi-4$ th th 45 0 ot in th this $\infty$ med in: in wh in m 0000 m $m 00 \%$ in $\mathrm{rm}$ on 0 $m M 000$

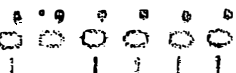

on is in in ton - $\infty$ in in in in min un un th $0 m=0 \%$ $m m m$ m $\rightarrow \ln 0$ ind on $m=000$

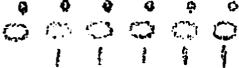

and rist

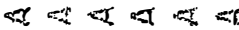

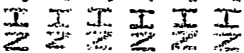
mol twi ind $m$

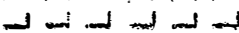

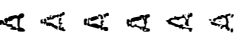

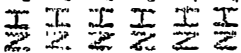

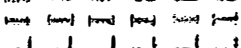




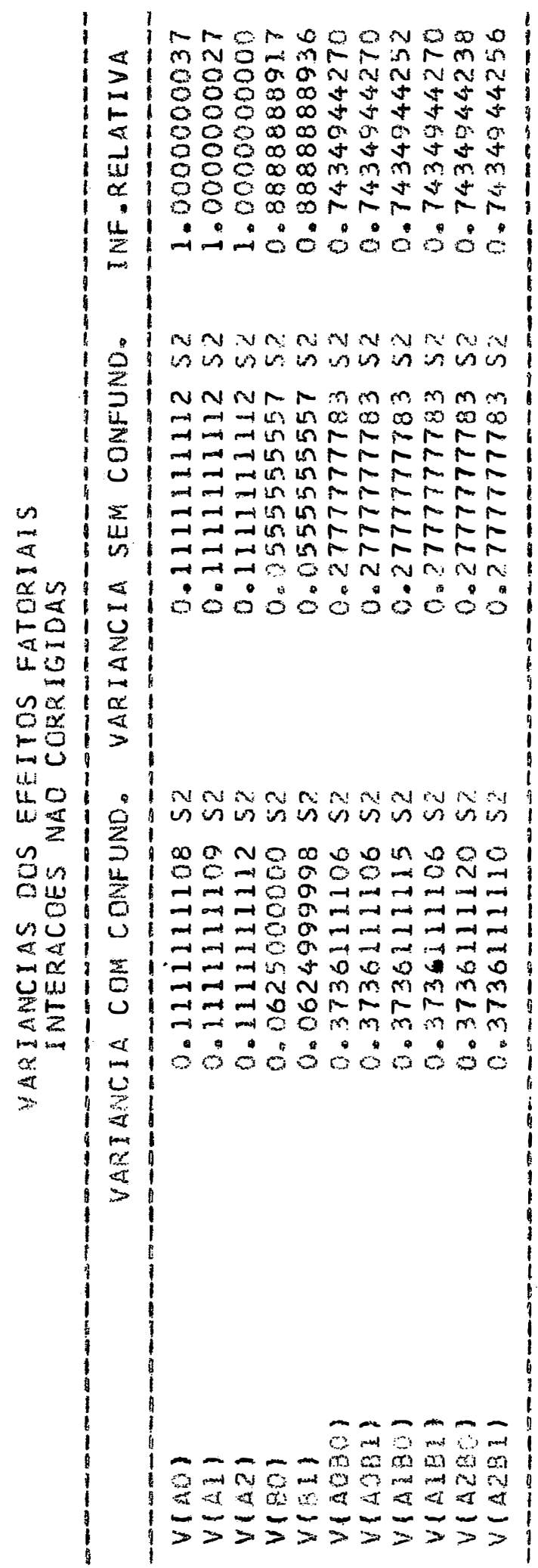

